\title{
Face Masks in the New COVID-19 Normal: Materials, Testing, and Perspectives
}

\author{
Ming Hui Chua $\mathbb{D}^{1},{ }^{1}$ Weiren Cheng $\mathbb{D},{ }^{1}$ Shermin Simin Goh $\mathbb{D},{ }^{1}$ Junhua Kong $\mathbb{D},{ }^{1}$ Bing Li, ${ }^{1}$ \\ Jason Y. C. Lim $\mathbb{D}^{1},{ }^{1}$ Lu Mao $\mathbb{D}^{1},{ }^{1}$ Suxi Wang, ${ }^{1}$ Kun Xue, ${ }^{1}$ Le Yang $\mathbb{D}^{1},{ }^{1}$ Enyi Ye, \\ Kangyi Zhang $\mathbb{D},{ }^{1}$ Wun Chet Davy Cheong, ${ }^{1}$ Beng Hoon Tan, ${ }^{1}$ Zibiao Li, ${ }^{1}$ Ban Hock Tan $\mathbb{D},{ }^{2}$ \\ and Xian Jun Loh ${ }^{1}$ \\ ${ }^{1}$ Institute of Materials Research and Engineering, Agency for Science, Technology and Research (A*STAR), 2 Fusionopolis Way, \\ Innovis, Singapore 138634 \\ ${ }^{2}$ Department of Infectious Disease, Singapore General Hospital, Singapore
}

Correspondence should be addressed to Xian Jun Loh; lohxj@imre.a-star.edu.sg

Received 28 May 2020; Accepted 16 July 2020; Published 7 August 2020

Copyright ( 2020 Ming Hui Chua et al. Exclusive Licensee Science and Technology Review Publishing House. Distributed under a Creative Commons Attribution License (CC BY 4.0).

\begin{abstract}
The increasing prevalence of infectious diseases in recent decades has posed a serious threat to public health. Routes of transmission differ, but the respiratory droplet or airborne route has the greatest potential to disrupt social intercourse, while being amenable to prevention by the humble face mask. Different types of masks give different levels of protection to the user. The ongoing COVID-19 pandemic has even resulted in a global shortage of face masks and the raw materials that go into them, driving individuals to self-produce masks from household items. At the same time, research has been accelerated towards improving the quality and performance of face masks, e.g., by introducing properties such as antimicrobial activity and superhydrophobicity. This review will cover mask-wearing from the public health perspective, the technical details of commercial and home-made masks, and recent advances in mask engineering, disinfection, and materials and discuss the sustainability of mask-wearing and mask production into the future.
\end{abstract}

\section{Introduction}

Emerging and reemerging infections have emerged as a threat to human health in recent decades [1]. Given how interconnected the world is today, a pathogen capable of human-to-human transmission can spark an outbreak far from where it originated. The virus causing the Middle East Respiratory Syndrome, for example, emerged in the Middle East but caused an outbreak in Korea. The world is in the midst of the COVID-19 pandemic, which is caused by the SARS-CoV-2 virus. Lockdowns and travel restrictions imposed to halt the spread of COVID-19 have led to devastating economic repercussions. The control of an infectious disease is based on knowledge of its mode of transmission. The recent COVID-19 pandemic is caused by the novel coronavirus, SARS-CoV-2, which is transmitted largely by the respiratory route (vide infra) $[2,3]$.

The best nonpharmaceutical interventions against disease spread via the respiratory route are broadly termed social or safe distancing measures, i.e., reducing close contact between individuals $[4,5]$. Where safe distancing is not possible, personal protective equipment (PPE) is the accepted mode of self-protection. Masks and respirators are arguably the most important piece of PPE. They are a physical barrier to respiratory droplets that may enter through the nose and mouth and to the expulsion of mucosalivary droplets from infected individuals $[6,7]$. Their role may be particularly important in COVID-19, where infected individuals may be shedding virus while asymptomatic or presymptomatic [8-10].

There are many different types of face masks and respirators offering different levels of protection to users [11-15]. Generally, masks do not fit tightly while respirators do. Masks and respirators may be reusable or disposable. Reusable ones include industrial-use half or full facepiece respirators with cartridge filters attached and homemade or commercial cloth masks; disposable ones include surgical masks, N95 respirators, and KN95 respirators. They all serve the general purpose of providing some form of protection against contaminants in 


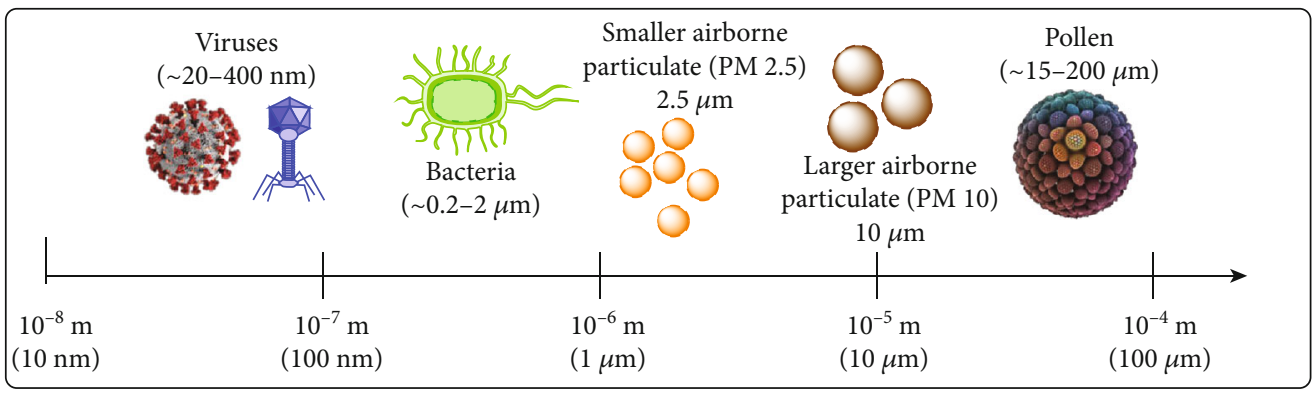

FIGURE 1: Relative size chart of common airborne contaminants and pathogens.

the air, ranging from pollen to chemical fumes to pathogens. The filtering capacity, and hence the level of protection against pollutants and pathogens, depends on the materials used and the engineering design [11-15]. Contaminants in the air differ vastly in size (Figure 1). SARS-CoV-2 has a size ranging from 60 to $140 \mathrm{~nm}$ [16], smaller than bacteria, dust, and pollen. Therefore, masks and respirators made of materials with larger pore sizes, such as cotton and synthetic fabric, will not be able to effectively filter these viruses or tiny virus-laden droplets, as compared with those made of materials with much smaller pore sizes. Likewise, masks and respirators made of or coated with water-resistant materials are more effective against large virus-laden respiratory droplets and fluid spills. In addition to filtering capacity, factors such as user comfort and breathability also vary across different models. For instance, although the tight-fitting N95 respirator has filtering capacity superior to surgical masks, they have lower breathability and may cause discomfort after hours of wearing.

Mask-wearing can be effective in the containment of communicable diseases $[17,18]$ and has thus become a new normal in many societies in the COVID-19 pandemic. The surge in demand for surgical masks and respirators has led to a global shortage of supply and raw materials. As a result, many people have resorted to making their own masks, recycling used masks, or settling for masks offering less protection than actually needed. Researchers and industry players have therefore been working hard to address the issue of shortage, as well as to enhance the protection afforded by existing mask models. These efforts include (i) sourcing and engineering alternative materials with sufficient filtering capacity, (ii) engineering the design of masks and respirators for better protection, breathability, and user comfort, (iii) developing and engineering multifunctional masks and materials with hydrophobic, antimicrobial, self-disinfecting, and even sensing properties, and (iv) exploring new technologies for efficient production and customization of masks, e.g., 3D printing [19].

Attempts to enhance the mask will pivot on understanding the basics of mask technology. The fundamental questions, to our mind, are as follows: (i) how do masks (and the mask materials) protect us from pathogens; (ii) what are the existing models and materials of mask available in the market; (iii) how do they perform and how is their performance benchmarked against others; (iv) what are their limitations; (v) how can their performance be improved; (vi) what are some new features that can be incorporated into existing materials and models? This review seeks to address the above questions.

\section{How Do Masks Protect Us against Airborne Diseases}

2.1. The Respiratory Route of Transmission. A respiratory pathogen may be transmitted via three routes-contact, droplet, and airborne spread [20]. Contact transmission may be direct (i.e., transfer of virus via contaminated hands) or indirect (i.e., via fomites) [20]. Fomites are objects or materials that may carry infection, and spread by fomites means spread by touch. Viruses do survive for some time on inanimate objects, although the viral load declines dramatically [21]. If we touch a contaminated surface and then touch our eyes or nose, we may inoculate the virus into our mucosal surfaces. The role of touch in the spread of a respiratory virus is best exemplified by studies of the Respiratory Syncytial Virus (RSV) $[22,23]$. The spread of SARS-CoV-2 via fomites has been elegantly demonstrated by real-world contact tracing, aided by closed-circuit cameras [24].

Droplet spread and airborne spread are different modes of transmission of the virus through the air. Viruses released when an infected person coughs, sneezes, sings, talks, or merely exhales may be found in particles of varying sizes [17]. Generally, particles larger than $5 \mu \mathrm{m}$ were thought to fall to the ground within 1 metre. More recently, however, the "gas cloud" hypothesis has been proposed [25]. Coughing, sneezing, or even exhaling produces mucosalivary droplets that exist as part of a cloud that "carries within it clusters of droplets with a continuum of droplet sizes" [25]. In combination with environmental factors, the "cloud" may be propelled up to $7-8 \mathrm{~m}$. Wind speed, in particular, has been shown to play a role in determining the distance travelled by these particles [26].

Airborne spread occurs with pathogens found in exhaled droplets $<5 \mu \mathrm{m}$ in diameter. These particles remain afloat for some time and are able to travel long distances. Respiratory viruses accepted as being capable of spread via the airborne route include measles and varicella zoster (chickenpox). These viruses have a large $R_{0}$, a feature thought to characterise spread by the airborne route. Interestingly, influenza, coronavirus, and rhinovirus RNA, generally thought to be transmitted by the droplet route, can be found in exhaled particles smaller or larger than $5 \mu \mathrm{m}[17,27]$. Further, viable influenza is present in particles smaller than $5 \mu \mathrm{m}$. Hence, even viruses thought to be transmitted primarily by the respiratory droplet route may have the potential for airborne spread. Concern that SARS-CoV-2 may spread by the airborne route rose when it 
was shown to be viable for 3 hours in a drum that artificially kept particles afloat for several hours [21].

It might be less well known that more basic processes like talking can also lead to the release of potentially infectious droplets and aerosols. Using laser light scattering, it was found that there were average emissions of about 1000 droplet particles per second during speech, with high emission rates of up to 10,000 droplet particles per second [28]. By fitting the timedependent decrease in particle detected to exponential decay times, the droplet particle sizes and estimated viral load could be calculated. The authors estimate that $1 \mathrm{~min}$ of loud speaking generates greater than 1000 droplets containing viruses [29]. Alternatively, respiratory particles of between $0.5 \mu \mathrm{m}$ and $5 \mu \mathrm{m}$ could be imaged by aerodynamic particle sizing. When participants made the "Aah" sound, there were emissions of up to 330 particles per second [30]. Taking into account that aerodynamic particle sizing measures particles under the detection limit of laser light scattering, these two methods can be seen to be complementary, and the total number of particles emitted could be even higher. In a separate study, droplet particle emission was shown to be directly proportional to loudness, with the number of particles emitted increasing from 6 particles per second when whispering to 53 particles per second at the loudest talking. The number of particles generated varied greatly across individuals, raising the possibility of superspreaders who could be the primary spreaders of viruses by talking [31].

2.2. Mechanistic Effect of Wearing a Mask. Masks and other PPE items serve as a physical barrier to respiratory droplets. With imaging using laser light scattering, it was found that the number of flashes, which corresponds to the number of respiratory droplets, could be kept at background levels by covering the speaker's mouth with a slightly damp washcloth [28]. An in vitro model with source and receiver mannequins was created to test the effect of the mask on filtering away radiolabelled aerosol emitted from the source. Masking at the source mannequin was consistently more effective at lowering radio-labelled aerosols reaching the receiver mannequin, whereas the only experimental setup where the receiver mannequin could be equally well protected was if the receiver mannequin wore an N95 mask sealed with Vaseline [32]. Therefore, masks can act as a physical barrier and seem to be more effective when worn by the droplet emitting person.

Masks have generally shown an effect in reducing virus emission from infected patients. The surgical mask was tested for its ability to block the release of various viruses by studying the amount of virus present in the exhaled breath of patients. The investigators were able to collect particles separated by size ( $>$ or $<5 \mu \mathrm{m}$ ). A significant drop in coronaviruses in both larger and smaller particles was observed with the mask on. The mask reduced influenza viruses found in larger but not smaller particles. After wearing a mask, no coronavirus was detected in all 11 patients, while influenza was detected in 1 patient's respiratory particles (out of 27). The mask did not lower rhinovirus counts in larger or smaller particles [17]. This suggests that surgical face masks can reduce the release of coronavirus and influenza from an infected person. In an earlier study for influenza, participants were induced to cough, and with both surgical masks and N95 masks, there was no influenza that could be detected by reverse transcriptase-polymerase chain reaction (RTPCR) for 9 infected patients [33]. When the exhaled influenza virus was separated into the fractions based on size, it was found that surgical masks were highly effective at removing influenza from the larger coarse fraction $(\geq 5 \mu \mathrm{m})$ but less effective from the fraction with smaller particles [34].

Wearing masks has also been shown to protect individuals coming into contact with an infected person. In a survey of 5 hospitals in Hong Kong during SARS, hospital staff were asked about the protective measures they took and this information was correlated with whether they were infected by SARS. It was found that wearing masks was the single most important protective measure in reducing the chance of getting infected $(p=0.0001)$, and the people who wore either surgical masks or N95 masks were not among the 11 infected staff. There were however 2 instances of people who wore paper masks being infected, suggesting that the type of masks was also important [35]. A study compared the effectiveness of N95 and surgical face masks against viral respiratory infections in healthcare workers. Healthcare workers had no significant difference in influenza infection outcomes when wearing N95 and surgical masks, suggesting that both types of medical masks could protect similarly [36]. A metaanalysis was performed on clinical studies to explore the protective effect of masks. The risk ratio was calculated for the incidence of infection in the protected group vs. the unprotected group, where risk ratio $<1$ suggests a reduced risk. Wearing a mask protected individuals against influenza-like illness, showing a risk ratio of 0.34 , with a $95 \%$ confidence interval between 0.14 and 0.82 . Similar to the study above, surgical masks and N95 masks showed little difference in protection, with a risk ratio of 0.84 and a $95 \%$ confidence interval of 0.36-1.99 suggesting no significant difference in risk [37].

Recently, a modelling study performed by Eikenberry et al. based on COVID-19 infection data obtained in New York and Washington suggested that the broad adoption of face mask by the general public can significantly reduce community transmission rate and death toll [18]. As shown in Figure 2, based on data obtained from $20^{\text {th }}$ February to $30^{\text {th }}$ March, the cumulative death rate was projected to be reduced to a greater extent as more people wear masks over the next 2 months. Therefore, the study concludes that communitywide adoption of face mask has great potential to help curtail community transmission and the burden of the COVID-19 pandemic.

2.3. Advantages and Caveats of Wearing Masks. Mask usage, in addition to other nonpharmaceutical interventions, can be an effective containment measure in an epidemic. Face masks can prevent dispersal of droplets when infected persons talk, sing, cough, or sneeze. The rate of emission of particles correlates with voice loudness during speech or other vocal activities [38]. A physical obstruction that prevents the wearer from touching the face, a mask may lead to better hand hygiene [24]. The reverse is also true-an increased tendency for wearers to touch their faces, such as when 

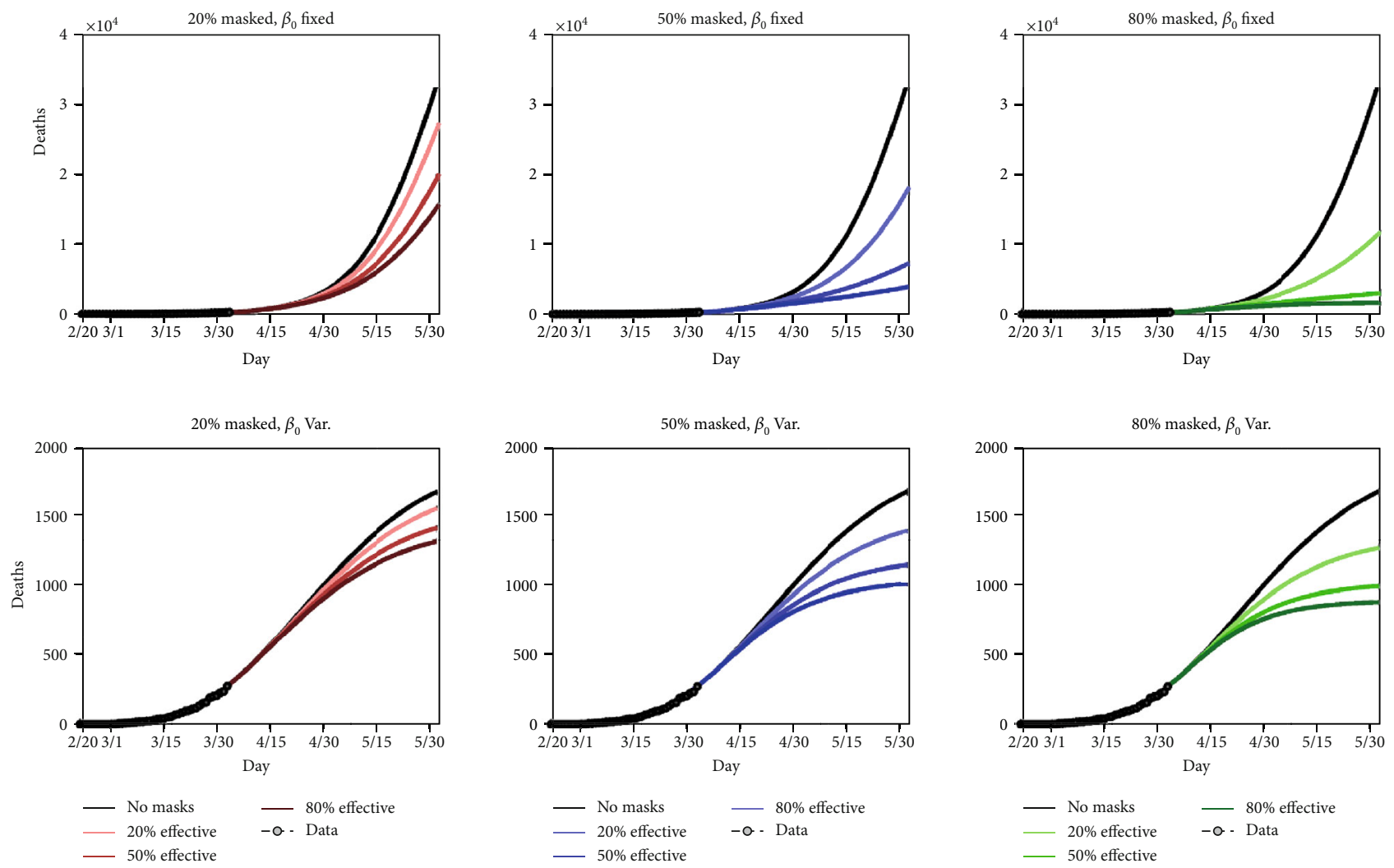

Figure 2: Simulated future (cumulative) death tolls for Washington state, using either a fixed (top panels) or variable (bottom panels) transmission rate, $\beta$, and nine different permutations of general public mask coverage and effectiveness. The $y$-axes are scaled differently in the top and bottom panels. Reproduced with permission from Ref. [18]. Copyright 2020, Elsevier B.V. on behalf of KeAi Communications Co., Ltd.

adjusting their masks [39]. Even with the right mask, wearers can still be infected if droplets enter via the eyes, thus highlighting the importance of additional protection [40].

Masks also reduce the risk of environmental contamination by respiratory droplets [24]. As mentioned, SARS-CoV2 transmission via fomites has been documented [24]. In reality, usage by each individual varies. The mask may not fully cover the mouth and nose, or it may be used and reused too frequently. These can mean huge variations in mask performance outcomes [24, 41]. In addition, wearers should avoid touching their faces and the external surface of their masks. Hand hygiene also varies from person to person. Hence, mask usage must be complemented by other behavioral changes for effective infection prevention. Finally, the universal use of face masks prevents discrimination of individuals who wear masks when unwell because everybody is wearing a mask. Universal mask-wearing can create new social norms, motivating individuals to wear masks at the initial onset of symptoms without fear of being stigmatized. The unintentional infection of healthy individuals by asymptomatic and presymptomatic persons can be avoided [39, 40]. Masks are visible indicators of crisis mode, which can prompt behavioral changes such as social distancing and frequent handwashing [39].

2.4. Complications of Asymptomatic and Presymptomatic Transmission. In the early stages of the COVID-19 pan- demic, many countries did not recommend mask-wearing by healthy people to prevent panic buying and stockpiling [41]. Such paranoia can lead to a drastic shortage of medical masks for healthcare workers. The efficacy of masks in protecting individuals from being infected was also doubted. Since then, governments, such as Singapore's, have made it mandatory to wear a mask in public [42]. Such a change of policy direction has come about primarily because of increasing recognition of the concept of asymptomatic and presymptomatic transmission.

There has been a rise in asymptomatic and presymptomatic cases reported in many parts of the world. In an early example of such transmission, five symptomatic patients contracted the virus from one asymptomatic relative who travelled from Wuhan to Anyang [43]. In the same time period, another family cluster of three travelling from Wuhan to Guangzhou revealed asymptomatic transmission [44]. While the adult male presented clinical symptoms, his wife and son were both asymptomatic. All three tested positive for COVID-19 on RT-PCR. A separate study on 82 residents at a long-term care skilled nursing facility revealed that out of the $30.3 \%$ positive cases, $43.5 \%$ were symptomatic while more than half were asymptomatic [45]. After 1 week, this asymptomatic group was reassessed and 10 out of 13 developed symptoms, leading to their reclassification as presymptomatic. Likewise in Singapore, 243 cases comprising of seven clusters could be explained by presymptomatic 
transmission [38]. In four of these clusters, the exact date of transmission could be determined to lead to the conclusion that transmission occurred 1-3 days before symptoms appear in the source patient. These cases demonstrate that viral shedding can occur prior to the onset and absence of symptoms, thus complicating the containment of this COVID-19 pandemic. Various governments have to enforce social distancing, good hygiene practices, and mask usage to effectively contain asymptomatic and presymptomatic transmission.

2.5. Household Mask Usage. It is a lot more ambiguous when it comes to household mask usage. What we do know is that wearing a mask or protective covering can reduce the emission of droplets and infectious viruses from the infectious person $[17,32,40]$. Laser light scattering studies revealed that covering the mouth of a speaker with damp cloth reduced particles emitted to background levels. Therefore, wearing a cloth mask or even a scarf, as recommended by the Centers for Disease Control and Prevention (CDC), to cover the nose and mouth would serve to reduce respiratory emissions from an infected person, whether he is symptomatic or asymptomatic [46].

The type of mask material worn is important in mitigating the risk of infection. For instance, a study found that in a healthcare setting, the risk of influenza was substantially higher in the cloth mask group than the medical mask group [47]. Hence currently, surgical face masks and N95 respirators are still the best option, if available, for protecting a healthy person in a high-risk environment [35]. A more comprehensive experimental investigation on the protective effect of reusable cloth masks is urgently required, particularly during an extended pandemic period when a sustainable low-cost option is essential for household usage.

Studies on the effect of wearing face masks in households have been plagued by confounding factors and adherence issues. While face masks and hand hygiene have been known to be key protective measures against droplet and fomite transmission, a study of 259 households in Hong Kong showed no significant difference in the infection risk for the group that both wore face masks and observed hand hygiene [48]. A similar conclusion was reached among young adults living in university residence halls, where a combination of face masks and hand hygiene did not correlate with a significant decrease in the rate of influenza-like illness [49]. A separate study provided some insight into the underlying reason. While there was no significant decrease in infection risk by wearing face masks, it was found by self-reporting that less than $50 \%$ of study participants wore masks most of the time. In the group that wore masks most of the time, it was found that the risk of infection decreased, with a risk ratio of 0.26 , with a $95 \%$ confidence interval of 0.06 to 0.77 [50]. Face mask-wearing in households seems to be ineffective for seasonal infectious diseases. Whether or not mask-wearing at home should be regulated or recommended is doubtful, since household members will necessarily eat together, an activity during which masking is impossible. Normalcy at home is critical to mental health in a pandemic.
2.6. Public Policy and Population-Level Effects. As discussed, asymptomatic and presymptomatic cases have made pandemic containment increasingly challenging, resulting in a paradigm shift in government approaches. Undocumented cases, many of whom were asymptomatic or mildly symptomatic, were possibly contributing to a large number of infections (79\%) in China [51]. A random sampling of $3000 \mathrm{New}$ York residents at various locations, such as grocery stores, revealed an infection rate of $13.9 \%$, and an estimated 2.7 million people might have been infected [52]. On 6th April, an interim guideline from the WHO stated that healthy people did not need to wear masks because there was no evidence that masks can protect the wearers [53]. This contrasted with CDC guidelines on 3rd April, which recommended cloth face coverings in public spaces, especially where there is significant community-based transmission [46].

Anecdotal evidence within hospital settings showed that universal mask usage must be implemented in high-risk areas. Symptoms of COVID-19 are similar to other respiratory diseases, and some healthcare workers displaying mild symptoms continue to work [39]. In another multiyear single-center study, a mask-wearing policy was instituted for all who interacted with hematopoietic stem cell transplant patients. Comparing the mask and premask years, respiratory viral infections decreased significantly after the mask policy. Hospital-wide and in an adjacent hematologic malignancy unit, the absence of mask policy meant that infections remained high [54].

A 2003 SARS study on five Hong Kong hospitals revealed that staff who adopted all four measures of masks, gloves, gowns, and handwashing remained healthy. Staff who omitted at least one of these practices became infected but the wearing of masks was the most significant and important measure [35]. The other three measures conferred no additional significant protection to mask wearers. Hence, stopping droplet transmission at the face level is critical.

Mathematical modelling on the 2009 (H1N1) influenza concluded that if masks were enforced early at 100 versus 1000 infectious people, the severity of an outbreak could be reduced markedly [55]. Everyone, not only infectious individuals, must wear masks to significantly reduce the cumulative number of cases. In this model, the effectiveness of surgical masks was low and insignificant. For N95 respirators operating at $20 \%$ effectiveness, a significant reduction of influenza (20\%) was achieved if only $10 \%$ of the population wore them. If $25 \%$ and $50 \%$ of the population complied, the reduction became $30 \%$ and $36 \%$, respectively.

For COVID-19, similar conclusions were achieved in a theoretical model and empirical data set study [56]. Monte Carlo simulation and an SEIR (susceptible-exposed-infectious-recovered) model were used. When a minimum of $80 \%$ of people wore masks, the impact on the pandemic was significant and the curve flattened. However, this intervention failed when $50 \%$ or less of the population wore masks. By day 50 of a regional outbreak at the latest, universal masking could prevent widespread transmission. If enforced at day 75 with a $90 \%$ masking adoption, there was no impact on the spread of infection, highlighting the 


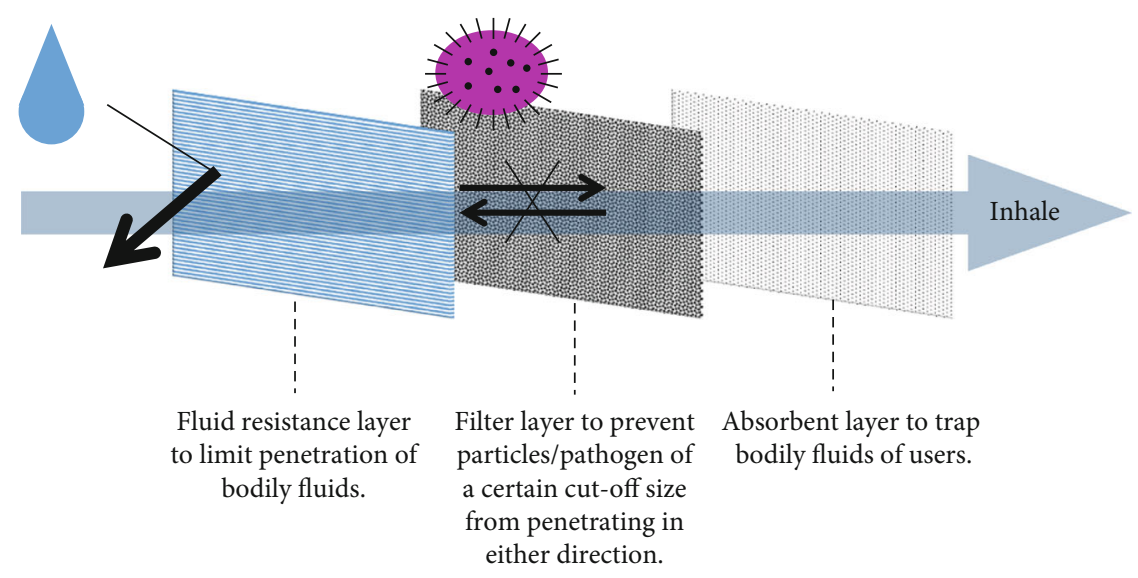

FIGURE 3: Illustration showing the function of each individual layers of a 3-ply surgical mask.

importance of early masking intervention. If at least $80 \%$ of the population wore masks, the curve could flatten more significantly than enforcing a strict lockdown. In addition, after lockdown, allowing social distancing without masking led to uncontrollable rise in infections.

With the results of these two simulations in mind, the authors studied the impact of masking enforcement on infection growth across many countries [56]. Countries can be classified into three tiers from best- to worst-performing. Across the tiers, the average daily infection growth rate and reduction from the peak are as follows: top at $5.9 \%$ and $74.6 \%$, middle at $14.2 \%$ and $45.8 \%$, and low at $17.2 \%$ and $37.4 \%$. Best-performing countries instituted universal masking orders before 15th March while those in the middle tier did this after the date. The remaining countries were the worst-performing. Overall in every region, employing universal masking resulted in better management of COVID-19.

Even though the Hong Kong government recommended mask usage only for symptomatic people, as with WHO guidelines, the general public volunteered to wear mask proactively [24]. Universal masking was also advocated by leading experts in clinical microbiology and infectious disease specialties. After 100 days, the number of infected per million population in Hong Kong was significantly lower than countries without universal masking. The comparison was done for countries with similar population density, healthcare system, BCG vaccination, and social distancing measures. These countries include Singapore, South Korea, Spain, Italy, and Germany. While infections and deaths have skyrocketed worldwide, Hong Kong has recorded low numbers, particularly remarkable given its close proximity to China and high population density. The main difference causing the favourable outcome in Hong Kong was the voluntary universal masking among residents since early in the pandemic. By observation of morning commute over three consecutive days, only $3.4 \%$ out of 10,050 persons failed to wear masks. There were eleven COVID-19 clusters which could be attributed to recreational mask-off settings such as dining and drinking in restaurants or bars, singing at karaoke parlours, and exercising in gymnasiums.

Therefore, these experiments and population studies show that universal masking is effective if implemented early and rigorously. Governments should deploy resources to obtain sufficient masks in order to achieve sustainable universal masking. If supplies are insufficient, the general public should use cloth masks when they are outside their homes. Medical masks should be reserved for healthcare workers and others who perform essential functions [56].

\section{Understanding Performance of Commercial Mask}

3.1. 3-Ply Surgical Mask. The 3-ply surgical mask is commonly used in the COVID-19 pandemic. The 3-ply surgical mask is made up of 3 different layers of nonwoven fabric with each layer having a specific function, as shown in Figure 3. The outermost layer (typically blue) is waterproof and helps to repel fluids such as mucosalivary droplets. The middle piece is the filter, which prevents particles or pathogens above a certain size from penetrating in either direction. The innermost layer is made of absorbent materials to trap mucosalivary droplets from the user. This layer also absorbs the moisture from exhaled air, thus improving comfort. Together, these 3 layers effectively protect both the user and the surrounding people by limiting the penetration of particles and pathogens in both directions.

As suggested by its name, nonwoven fabric does not contain intertwining strands and is made by bonding a mass of fibres together using heat, chemical, or mechanical means. Felt is one of the most common examples of nonwoven fabric. Although nonwoven fabric is mechanically weaker than its counterpart, it is cheap and fast to manufacture. Therefore, it is an ideal material for the surgical mask. The two most common methods of making nonwoven fabric for surgical mask are spunbond and melt-blown.

The spunbond process combines the spinning and sheet formation process into one continuous, nonwoven manufacturing system $[57,58]$. As seen in Figure 4, the spunbond process consists of several integrated steps, namely, extruder, gear pump, spinpack, quencher, collector, bonder, and winder.

(i) Extrusion is the process where the polymer is melted by heat and mechanical action of the screw 

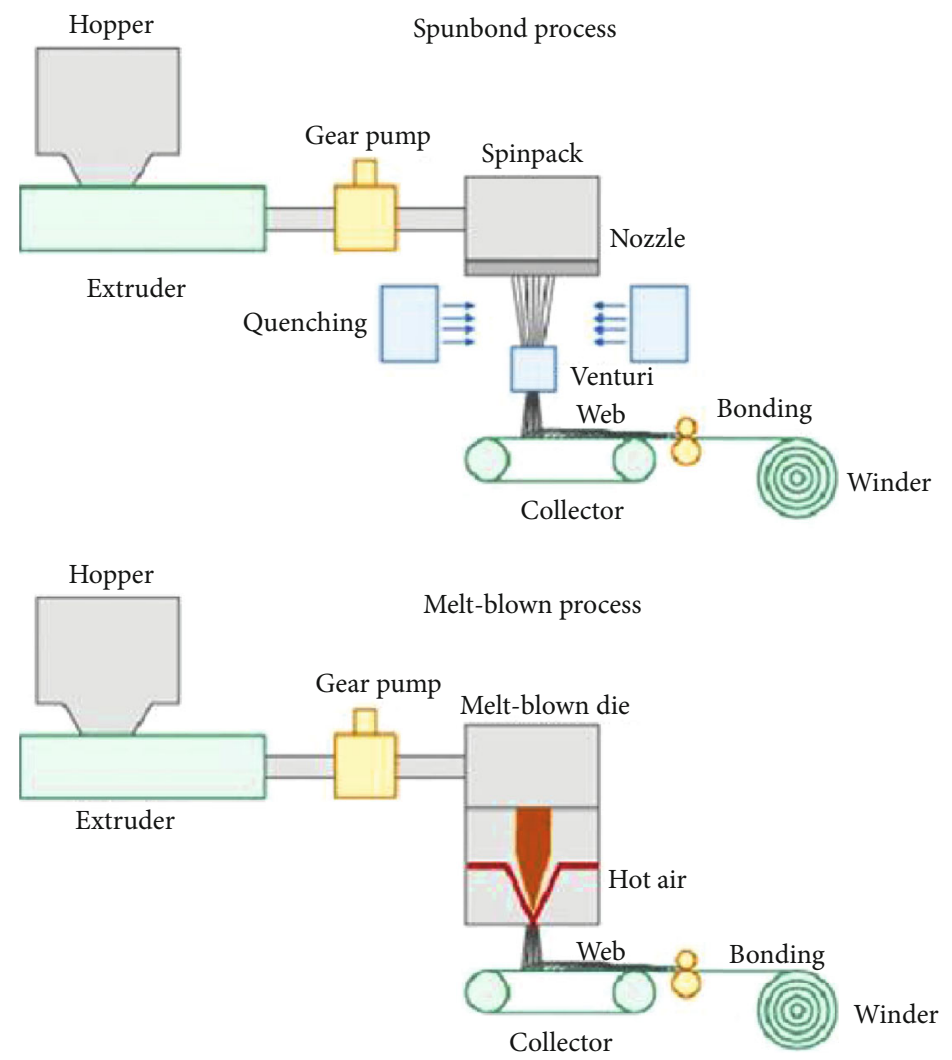

Figure 4: Schematic illustration of spunbond and melt-blown process. Republished with permission from Ref. [59]. Copyright 2015, Butterworth-Heinemann.

(ii) The gear pump plays a critical role in controlling the precise volumetric flow rate of the molten polymer. This is a key step to maintain a uniform temperature of the molten polymer

(iii) The spinpack is a die block assembly which turns the molten polymer into uniform thin filaments and is designed to be able to withstand $300^{\circ} \mathrm{C}$ to $400^{\circ} \mathrm{C}$

(iv) The filaments are then quenched by cool air

(v) After quenching, the filaments are collected together as filament web on a moving belt

(vi) The filaments in the web are then bonded together via heat, chemical, or mechanical means to form the nonwoven fabric

(vii) Lastly, the nonwoven fabric is collected in the winder

Although the melt-blown process is very similar to the spunbond as seen in Figure 4, the microfibres produced in melt-blown are much finer and the pore size of the nonwoven fabric can be much smaller. Therefore, due to the finer pore size, melt-blowing is the typical process used to fabricate the middle filtering piece of the 3-ply surgical mask. The melt-blown process also consists of several integrated steps, namely, extrusion, gear pump, die assembly, collector, and winder [60]. The major difference between spunbond and melt-blown is in the die process which is the most important element responsible for the smaller diameter microfibres. There are three components in the die assembly: the feed distribution plate, die nosepiece, and air manifold which are all kept heated at $215^{\circ} \mathrm{C}$ to $340^{\circ} \mathrm{C}$.

(i) The feed distribution plate ensures the molten polymer flows across the plate evenly. The shape of the feed distribution plays an important role in the polymer distribution. The most common, coat hanger-type, has a manifold at the polymer entrance to ensure even and uniform distribution of polymer flow

(ii) The die nosepiece is the key component which ensures the filament diameter and quality. The die tip is a very wide and thin metal piece with orifice measuring about $0.4 \mathrm{~mm}$. As a result, the die tip is very fragile and has to be replaced frequently once the metal between the orifices is broken

(iii) The air manifold, shown in Figure 5, supplies hot, high-velocity air which draws the polymer filaments into much thinner microfibres. The manifolds are unusually located at the side of the die nosepiece, and the hot air comes in contact with the polymer as it exits the die tip. The air is hot than the polymer to ensure the polymer remains liquefied during the process 


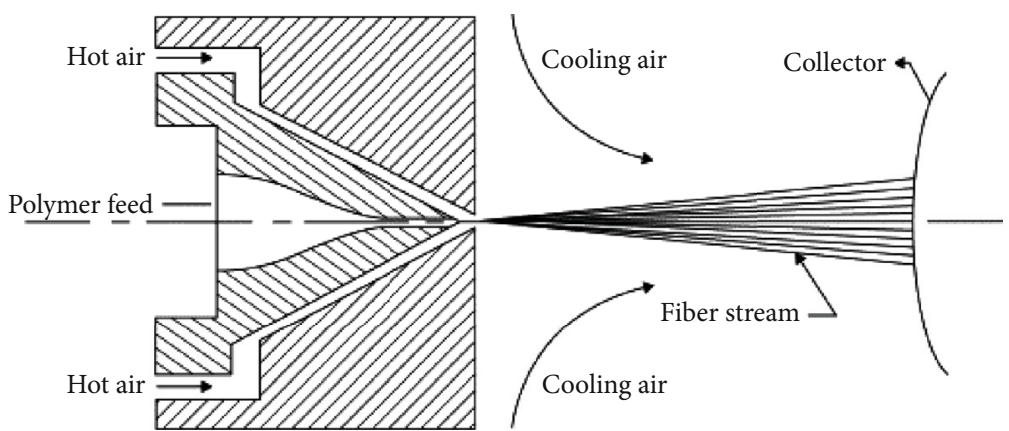

FIGURE 5: Schematic illustration of air manifold in melt-blown process. Republished with permission from Ref. [61]. Copyright 2014, Woodhead Publishing Limited.

Both the spunbond and melt-blown technologies are capable of processing a great variety of thermoplastic like polypropylene, polyester, polyethylene, polyamide, and polyurethane $[57,58,60]$. Of all materials, polypropylene is the most common as it is relatively cheap and has low melt viscosity for easy processing. Coincidentally, polypropylene is the most common material used for a 3-ply surgical mask while other materials like polystyrene, polycarbonate, polyethylene, and polyester can also be used in masks [62].

3.2. Air Flow Through Mask. To understand the flow of air through the mask, fluid mechanics is needed. Figure 6 illustrates how air flows through a narrow channel.

According to the Bernoulli equation,

$$
P_{1}+\frac{1}{2} \rho v_{1}^{2}+\rho g h_{1}=P_{2}+\frac{1}{2} \rho v_{2}^{2}+\rho g h_{2}
$$

where $P$ is the pressure, $\rho$ is the density of the fluid, $v$ is the velocity, $g$ is the gravitational constant, and $h$ is the height.

Under restrictions:

(1) Steady flow

(2) Incompressible flow

(3) Frictionless flow

(4) Flow along a streamline

Since the channel in Figure 4 is along the same horizon,

$$
\rho g h_{1}=\rho g h_{2} .
$$

Combining (1) and (2),

$$
\begin{aligned}
P_{1}+\frac{1}{2} \rho v_{1}^{2} & =P_{2}+\frac{1}{2} \rho v_{2}^{2}, \\
P_{2}-P_{1} & =\frac{1}{2} \rho v_{1}^{2}-\frac{1}{2} \rho v_{2}^{2}, \\
\Delta P & =\frac{1}{2} \rho\left(v_{1}^{2}-v_{2}^{2}\right), \\
\Delta P & =\frac{1}{2} \rho\left(v_{1}+v_{2}\right)\left(v_{1}-v_{2}\right) .
\end{aligned}
$$

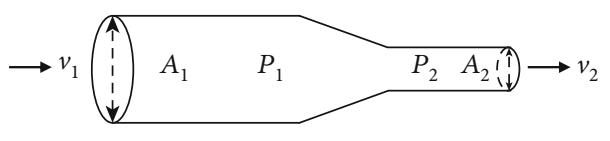

FIGURE 6: Air flows through a channel with different sizes.

Under the conservation of mass, the continuity equation ensures that the amount of mass entering the channel is equal to the amount of mass exiting the channel:

$$
\begin{aligned}
\rho A_{1} v_{1} & =\rho A_{2} v_{2}, \\
\frac{A_{1}}{A_{2}} & =\frac{v_{2}}{v_{1}},
\end{aligned}
$$

where $A$ is the area and $v$ is the velocity.

Therefore, when air flows through the channel in Figure 6,

$$
\frac{A_{1}}{A_{2}}=\frac{v_{2}}{v_{1}}>1 .
$$

When Equation (5) $>1$, the change in pressure, Equation (3), when the air flows through the channel in Figure 6 is

$$
\Delta P<0 .
$$

The airflow through the channel in Figure 7 illustrates how air will penetrate the mask whenever the user breathes in. Air from the surrounding environment is forced into the numerous tiny pores on the mask. From Equations (3) and (5), it is clear that the larger the difference in the areas the air flows through or the smaller is the mask pore, the greater the pressure drops or negative differential pressure. In other words, the more difficult it is for the wearer to breathe through the mask.

3.3. Performance Criteria for Commercial Masks. Face masks provide the user with protection against airborne particles, pathogens, secretions, and body fluids by physically filtering them from breathable air. According to the American Society of Testing and Materials (ASTM) F2100 standard, which specifies the performance requirements for materials used in medical face masks [63], five performance characteristics have been identified. These are particulate filtration efficiency 


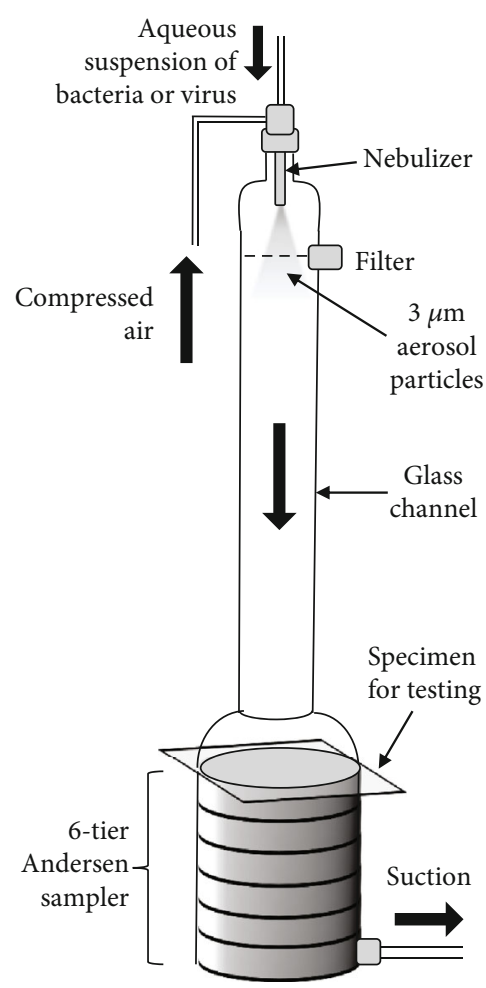

Figure 7: Design of the setup for evaluating BFE and VFE of mask materials using a six-stage Andersen sampler.

(PFE), bacterial filtration efficiency (BFE), fluid resistance, differential pressure, and flammability. As face masks are an integral part of the personal protective equipment (PPE) kit for medical use, these standardized characteristics ensure consistency in mask production and testing validation and help the end-user to make the most informed choice of mask for the intended application.

3.3.1. Particulate Filtration Efficiency (PFE). This test measures the filtration efficiency of face masks towards monodisperse particles under a constant airflow rate. For PFE testing, $0.1 \mu \mathrm{m}$ polystyrene latex particles are used according to FDA guidance [64] at airflow velocities of $0.5-25 \mathrm{~cm} / \mathrm{s}$ as recommended by the ASTM F2299 standard, for quantifying the filtration efficiency of materials used in facial masks [65]. Light scattering is used to quantify the particle count in the upstream feed $\left(M_{\mathrm{u}}\right)$ prior to filtration, as well as that in the downstream filtrate $\left(M_{\mathrm{d}}\right)$. The filtration efficiency $(E)$, often expressed as a percentage, can be calculated with Equation (1):

$$
E=100\left(1-\frac{M_{\mathrm{d}}}{M_{\mathrm{u}}}\right)
$$

The percentage of penetration $(P)$, or leakage of the particles through the mask medium, can hence be quantified by

$$
P=100\left(\frac{M_{\mathrm{d}}}{M_{\mathrm{u}}}\right)=100-E .
$$

It thus follows that the higher the value of $E$, with a corresponding smaller $P$, indicates a better ability of the mask material to filter submicron particles. While the F2299 standard allows consistent comparison of the PFE value of different materials used for face masks, it does not access the effectiveness of the overall design of the face mask, nor the quality of the mask's seal to the wearer's face.

3.3.2. Bacteria Filtration Efficiency (BFE). This test quantifies the performance of the mask material in filtering out bacteria when challenged with an aerosol of Staphylococcus aureus, as recommended by the ASTM F2101 standard [66]. S. aureus was chosen for its clinical relevance as one of the leading causes of nosocomial infections acquired in a hospital or healthcare facility $[67,68]$. To perform the test, an aerosolized liquid suspension of $S$. aureus (mean particle size of $3.0 \pm 0.3 \mu \mathrm{m}$ ) is delivered to the target filter sample at a constant flow rate of $1 \mathrm{ft}^{3} / \mathrm{min}$ (or $28.3 \mathrm{~L} / \mathrm{min}$ ). As shown in Figure 7 , the aerosol is then drawn through a six-stage Andersen sampler [69]. Each tier contains an agar plate which acts as a medium for the growth of any bacteria which passes through the filter material to form visible colonies on the plates. A control is also performed under identical conditions in the absence of the filter specimen. The percentage $\mathrm{BFE}$ can be calculated by the formula:

$$
\mathrm{BFE}=100\left(\frac{C-F}{C}\right)
$$

where $C$ and $F$ represent the number of bacteria colonies in the control and in the presence of the filter, respectively. Using the ASTM F2101 standard, a maximum BFE of 99.9 $\%$ can be achieved.

For surgical masks, a minimum BFE of $95 \%$ BFE is required. It should be noted that other than the ASTM specifications, some mask manufacturers quantify BFE ratings with the modified Greene and Vesley method [70], which measures the effectiveness of the mask in preventing bacteria from passing through when worn on a human test subject's face. This method is not comparable with ASTM F2101 and is not recommended by ASTM for comparison [71]. The ASTM F2101 method possesses numerous advantages, including a highly reproducible testing procedure, the ability to tightly control the mean bacteria aerosol particle size, and has not been modified for many years, which provides a consistent set of standards for comparing across many different filter materials assessed at different times [72]. However, like the ASTM F2299 standard for PFE, the ASTM F2101 standard for BFE does not evaluate the fit, design, and facialsealing properties of the mask.

3.3.3. Viral Filtration Efficiency (VFE). The viral filtration efficiency (VFE) is another parameter used by mask manufacturers for marketing and in FDA 510(k) applications for certain N95 filtering facepiece respirators [73], although it is not currently recognized as a standard test method by ASTM and hence is not a requirement for mask evaluation. The VFE test utilizes the same procedure and setup as 
recommended by ASTM F2101 for BFE (Figure 7) [72]. The bacteriophage $\Phi$ X174, which infects only E. coli bacteria, is used as the challenge virus that is aerosolized to form $3.0 \pm$ $0.3 \mu \mathrm{m}$ virus-containing water droplets (not individual viruses). Unlike the BFE test, the agar plates in the Andersen sampler are first inoculated with E. coli, and areas in contact with the viral droplets become clear as the bacteria cells are lysed to form plaques. The VFE value is calculated by comparison with a control without the filter material as described above for BFE.

3.3.4. Fluid Resistance. Fluid resistance evaluates the mask's ability to act as a barrier to the transfer of fluids from its outer to its inner layers due to spraying or splashing. According to the ASTM F1862 standard, $2 \mathrm{~mL}$ of synthetic blood, containing a red dye for visual detection and a thickening agent for stimulating blood flow properties, is dispensed against a complete medical mask specimen at different velocities [74]. These velocities correspond to different blood pressures of $80 \mathrm{mmHg}$ (Level 1, venous blood pressure), $120 \mathrm{mmHg}$ (Level 2, arterial pressure), and $160 \mathrm{mmHg}$ (Level 3, high pressures occurring during trauma or under surgical conditions with high-pressure irrigation) [71], assuming the face mask is within $300 \mathrm{~mm}$ of the blood vessel puncture. The pass/fail determinations are based on visually detecting penetration of the synthetic blood to the inner layer. To simulate actual usage conditions, i.e., breathing, which creates high humidity (thus affecting fluid resistance), and mask material, the test specimens are also preconditioned at high relative humidity of $(85 \pm 5) \%$ at $(21 \pm 5)^{\circ} \mathrm{C}$.

3.3.5. Differential Pressure (DP). This parameter, otherwise known as "delta P," measures the ability of the mask material to restrict airflow through it, giving an objective indication of the mask's breathability. Typically, it is determined by measuring the difference in air pressure on both sides of the mask material using a manometer at a constant airflow rate, and the difference in pressure is divided by the surface area of the sample, according to the MIL-M-36954 standard [75]. As such, DP is usually expressed in units of $\mathrm{mm} \mathrm{H}_{2} \mathrm{O} / \mathrm{cm}^{2}$, where a lower value (i.e., smaller difference in pressure on both sides) indicates greater breathability, feels cooler to the wearer, and hence gives an overall better comfort level. ASTM requires that moderate and high barrier masks have a DP value of $<5.0$, while low barrier masks have $\mathrm{DP}<4.0$. It is noteworthy that a trade-off exists between DP and fluid resistance for the same design and fit of the wearer: generally, an increase in resistance to synthetic blood penetration also results in a greater pressure drop across the mask layers and hence reduces breathability [74].

3.3.6. Flammability. Hospitals contain numerous sources of ignition, such as heat, oxygen, and fuel sources. As the natural and synthetic fibres making up the mask materials are flammable, these can pose potential risks to the wearer due to the speed and intensity of flame spreading. Mask flammability is assessed in accordance with the 16 CFR Part 1610 standard, typically performing the tests on 5-10 test samples [76]. In a nutshell, the mask specimen is first cut into the defined dimension of $50 \times 150 \mathrm{~mm}$, then mounted and secured onto a specimen holder. Thereafter, the mounted specimen is conditioned in a desiccating oven at $(105 \pm 3$ )$^{\circ} \mathrm{C}$ for 30 minutes, before it is then transferred to the test chamber. A stable butane flame of fixed length $(16 \mathrm{~mm})$ is then impinged upon the sample for exactly $1.0 \mathrm{~s}$. The burn time, i.e., the time taken for the flame to travel up the specimen till a stop device is triggered, is then registered. According to the ASTM F2100 Standard for Performance of Materials Used in Medical Face Masks [63], the masks need to meet the requirements of Class 1 flammability, with an average burn time of $\geq 3.5 \mathrm{~s}$ [77].

In addition to these aforementioned standardized tests, the medical face masks should be tested according to ISO 10993-5 and 10, which specifies cytotoxicity [78] and skin sensitivity [79] test methods, respectively, to ensure the materials are not harmful to the wearer. A summary of various mask types, their performance criteria, and use applications is provided in Table 1.

\section{Masks Made from Household Materials}

The surge in demand worldwide for commercial face masks during the COVID-19 pandemic has led to a global shortage of supplies for both physical products as well as raw materials [80]. In this circumstance, making a mask at home can be a life-guarding action [81]. Homemade masks may vary from the commercial ones in terms of structural integrity and effectiveness, but they are cheap and accessible. Wearing a simple cloth mask is far better than wearing no mask to safeguard the wearer and the others' health [81, 82].

Using commonly available household materials, it is easy to fabricate simple masks that may block respiratory droplets from the wearer. A lot of household materials have been used to fabricate masks and tested accordingly. These typically include cotton fabrics, clothing, silk, tissue paper, kitchen towels, pillowcase, and tea cloths. In the H1N1 influenza pandemic, researchers tested the efficiency of homemade masks against that of commercial masks. van der Sande et al. designed a series of experiments, including short-term (10-15 mins) inward protection, long-term ( $3 \mathrm{hrs)} \mathrm{inward}$ protection, and outward transmission prevention, to compare the effectiveness of three types of masks under different movement activities [14]. An N95-equivalent Filtering Facepiece against Particles- (FFP-) 2 mask $\left(1872 \mathrm{~V}^{\circledR}, 3 \mathrm{M}\right)$, a surgical mask (1818 Tir-On $^{\circledR}, 3 \mathrm{M}$ ), and a homemade mask made of TD Cerise Multi ${ }^{\circledR}$ tea cloths (Blokker) were chosen. In the short-term protection test, all masks gave some protection to both adults and children against airborne particles. FFP-2 provided the best protection to adults ( 25 times as much as a surgical mask and 50 times as much as a homemade mask from tea cloths), while the homemade mask provided the least protection. The protection to children was less efficient with all masks, though the efficiency ranks were the same as in adults. Activity (nodding, shaking, reading, and walking) had no obvious impact on efficiency. In the long-term protection test, the conferred protection remained highest with the FFP-2 mask and lowest with the homemade mask. Interestingly, the measured median protection factors increased with 


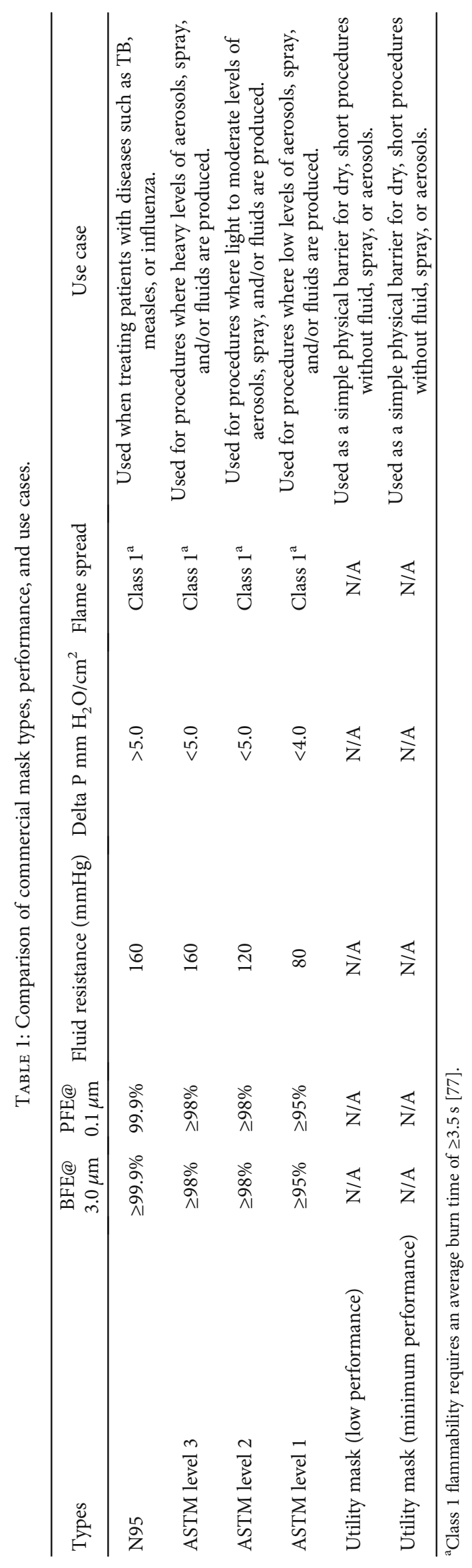


TABLE 2: The filtration efficiency and pressure drop across materials with two different microorganisms. Reproduced with permission from Ref. [12]. Copyright 2013, Cambridge University Press.

\begin{tabular}{|c|c|c|c|c|c|c|}
\hline \multirow[t]{2}{*}{ Material } & \multicolumn{2}{|l|}{ B. atrophaeus } & \multicolumn{2}{|l|}{ Bacteriophage MS2 } & \multicolumn{2}{|c|}{$\begin{array}{l}\text { Pressure drop } \\
\text { across fabric }\end{array}$} \\
\hline & Mean \% filtration efficiency & SD & Mean \% filtration efficiency & SD & Mean & SD \\
\hline $100 \%$ cotton T-Shirt & $69.42(70.66)$ & $10.53(6.83)$ & 50.85 & 16.81 & $4.29(5.13)$ & $0.07(0.57)$ \\
\hline Scarf & 62.30 & 4.44 & 48.87 & 19.77 & 4.36 & 0.19 \\
\hline Tea towel & $83.24(96.71)$ & $7.81(8.73)$ & 72.46 & 22.60 & $7.23(12.10)$ & $0.96(0.17)$ \\
\hline Pillowcase & $61.28(62.38)$ & $4.91(8.73)$ & 57.13 & 10.55 & $3.88(5.50)$ & $0.03(0.26)$ \\
\hline Antimicrobial pillowcase & 65.62 & 7.64 & 68.90 & 7.44 & 6.11 & 0.35 \\
\hline Surgical mask & 96.35 & 0.68 & 89.52 & 2.65 & 5.23 & 0.15 \\
\hline Vacuum cleaner bag & 94.35 & 0.74 & 85.95 & 1.55 & 10.18 & 0.32 \\
\hline Cotton mix & 74.60 & 11.17 & 70.24 & 0.08 & 6.18 & 0.48 \\
\hline Linen & 60.00 & 11.18 & 61.67 & 2.41 & 4.50 & 0.19 \\
\hline Silk & 58.00 & 2.75 & 54.32 & 29.49 & 4.57 & 0.31 \\
\hline
\end{tabular}

the wearing time for the homemade mask, while they decreased with the FFP-2. In the outward protection test, the mask type significantly determined the protection factors. The homemade mask only provided marginal outward protection, while the FFP-2 and the surgical mask, which performed similarly, provided better outward protection. Despite the relatively low effectiveness, it was suggested that wearing a homemade mask might sufficiently reduce viral exposure [12]. The marginal respiratory protection was also observed with masks made with other common materials including sweatshirts, T-shirts, towels, and scarves, when tested against polydispersed and monodispersed aerosols $(20-1000 \mathrm{~nm})$ [83]. Compared with the control N95 respirator, these fabric materials allowed higher penetration by aerosols, indicating poorer protection for wearers.

In addition to material, other factors, including the design, the velocity, the fitness to the wearer's face (sealing issue), and the properties of the particles to which it will be exposed, also affect the overall performance of a homemade mask. A more comprehensive study was conducted by Davies et al., to test the efficacy of homemade masks against bacterial and viral aerosols (Bacillus atrophaeus (B. atrophaeus) with a size of $0.95-1.25 \mu \mathrm{m}$, and bacteriophage MS2 with a size of $0.023 \mu \mathrm{m})$ [12]. The masks were made from different common household materials, including $100 \%$ cotton T-shirt, scarf, tea towel, pillowcase, antimicrobial pillowcase, vacuum cleaner bag, cotton mix, linen, and silk. As shown in Table 2, all materials are capable of blocking the microorganisms to different degrees, and they all worked better in the case of B. atrophaeus due to its large size. Although the surgical mask as a control sample possesses the highest efficacy, the vacuum cleaner bag, tea towel, and cotton mix also showed filtration efficiency of higher than $70 \%$. The ones with the lowest efficiency were the scarf, pillowcase, and silk, most of which however still had $>50 \%$ efficacy. Another important factor that needs to be considered when using a face mask is the ease of breathing, which is indicated by pressure drop. The higher the pressure drop, the higher the difficulty for the wearer to breathe. It is obvious that despite the high filtration efficiency of a vacuum cleaner bag and tea towel, their high- pressure drop values make them unsuitable for masks. Combining the above two factors, it was suggested that the most suitable household materials for a homemade mask are pillowcase and $100 \%$ cotton t-shirt, and further studies showed that doubling the layer did not help improve the efficacy significantly [12]. Yet, doubling increased the pressure drop, indicating more difficulty for breathing. This work again provides the insight that homemade masks are capable of blocking bacteria and viruses to some extent, yet their overall performance (filtration efficiency, pressure drop, and fitness) is not comparable to N95 and surgical masks. Indeed, wearing a mask can reduce the infection probability yet cannot eliminate the disease. It must be implemented communitywide [18] and together with multiple nonpharmaceutical preventative measures, such as hand hygiene, social distancing, quarantine, and immunization, to minimize the transmission and stop the outbreak [84]. In that sense, the homemade masks may be the last resort when facing a supply shortage, and they may well protect the general public.

Historically, cloth masks have been used to protect healthcare workers (HCWs) from respiratory infections [85-87], yet it is only in recent years that researchers started to systematically study their efficacy. Chughtai et al. reviewed the use of cloth masks [88] and conducted a series of studies including randomized clinical trial (RCT) to evaluate how good the cloth masks are to protect HCWs [47, 89]. Finding that the rate of respiratory infection was highest in the cloth mask group and that the particle penetration of cloth masks was $97 \%$ (versus $44 \%$ for medical masks), the authors concluded that cloth masks should not be recommended for HCWs, especially in highly infectious situations. Shakya et al. examined the efficiency of a cloth mask against monodispersed polystyrene latex (PSL) particles $(30 \mathrm{~nm}$ to $2.5 \mu \mathrm{m}$ ) and diluted whole diesel exhaust [90]. It was found that with an exhaust valve, the cloth mask had a filtration efficiency of $80-90 \%$ against PSL particles. Without a valve, the efficiency against the same PSL particles drops to $36-65 \%$, although the cloth mask performed better against larger particles. The cloth mask's filtration efficiency ranged from 15 to $75 \%$ against whole diesel particles. The overall performance 


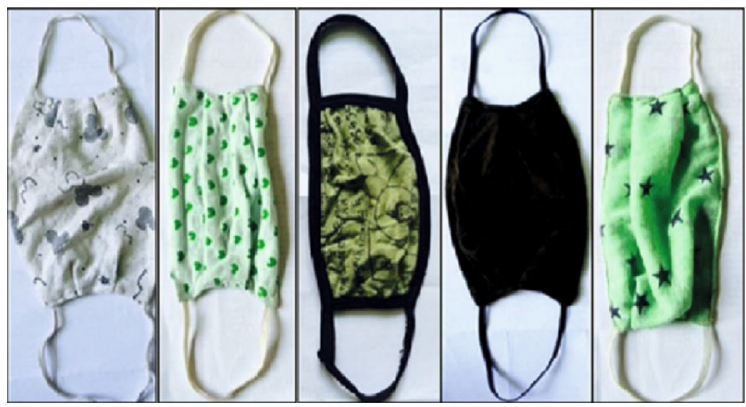

(a)

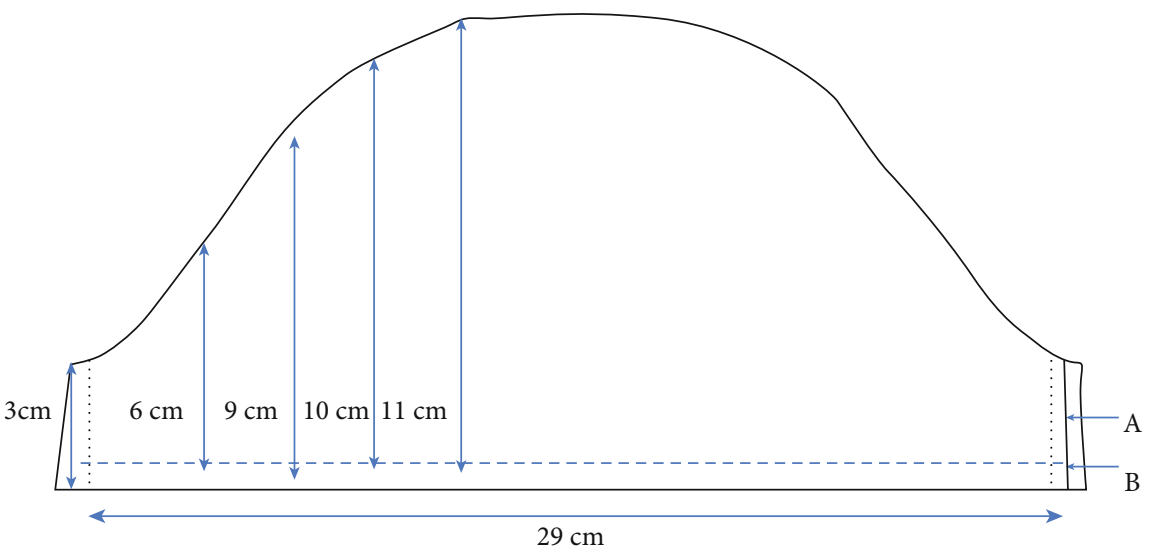

(b)

Figure 8: (a) Photos showing examples of simple cloth masks. Reproduced with permission from Ref. [91]. Copyright 2019, Neupane et al., Peer]. (b) Schematic showing the pattern for a homemade mask. Reproduced with permission from Ref. [92]. Copyright 2020, Springer Nature.

results suggested that cloth masks provided marginal protection to the wearer from particles less than $2.5 \mu \mathrm{m}$. Furthering the study on filtration efficiency, Neupane et al. investigated the effect of washing and drying of cloth masks on the filtration performance and correlated the performance to the pore size and shape in the masks [91]. It was found that the $\mathrm{PM}_{10}$ filtration efficiency dropped by $20 \%$ after the $4^{\text {th }}$ washing and drying cycle, which was ascribed to the increase in pore size and the lack of microfibres within the pore region. Longterm usage of the cloth masks entails continuous stretching of the mask, enlarging the pore size, thus impairing mask performance.

Making a cloth mask can be as simple as combining two cloth layers with stretchable ear loops (Figure 8(a)) [91]. Sugrue et al. introduced a step-by-step method of making a cloth mask using household materials including cotton, metal garden wire, and elastic bands (Figure 8(b)) [92]. The fabricated cloth mask has demonstrated its good comfort and fitness to the human face. While one can also find instructions on how to sew a fabric face mask at home [93, 94], Konda et al. recently developed a new model to fabricate homemade cloth masks that can achieve high filtration efficiency against aerosol particles ranging from $10 \mathrm{~nm}$ to $10 \mu \mathrm{m}$ in size [95]. By combining different commonly available fabrics, for instance, cotton-silk, cotton-chiffon, cotton-flannel, and filtration efficiency for particles $<300 \mathrm{~nm}$ and $>300 \mathrm{~nm}$ can be as high as $>80 \%$ and $>90 \%$, respectively. The high efficiency comes from the synergistic effect of mechanical filtration from cotton and electrostatic filtration

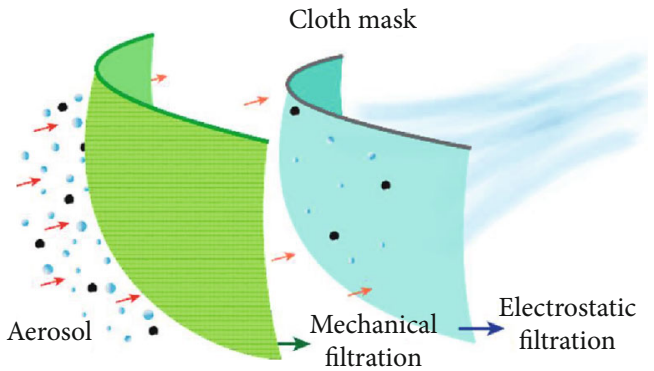

Figure 9: A new design of homemade cloth masks from common fabric materials. Reproduced with permission from Ref. [95]. Copyright 2020, the American Chemical Society.

from the other layer like silk (Figure 9). It was also highlighted in this work that for the same material such as cotton, there are other factors that critically and significantly affect the overall performance when used as a mask. These include the layer number, the layer density (threads per inch, TPI), and the facial fitness (openings and gaps between the mask edge and the facial contours). Therefore, future mask development should consider the above factors while taking into consideration the breathability, washability, and reusability.

\section{Decontamination of Face Masks}

The worsening and prolonging of the COVID-19 pandemic have led to a surge in daily consumption and demand of 
PPE items, including face masks, by frontline healthcare workers, which resulted in a global shortage of face masks and raw materials. Unfortunately, the production of masks cannot be easily ramped up to meet this sudden surge in demand. While reusable cloth masks purchased or selfmade from household materials can serve as a substitute for disposable surgical masks among the general population, such masks do not offer sufficient protection against the virus for healthcare workers who are working and in constant contact with infected patients for prolonged hours. In fact, these workers require the wearing of filtering facepiece respirators (FFR) such as the N95 masks as part of their PPE, which offer even more protection against airborne pathogens compared to the normal disposable 3-ply surgical masks. These masks are meant to be of one-time use, i.e., disposable, and are not recommended to be reused. The shortage of supply faced by many countries and hospitals, particularly those without sufficient stockpile, has however resulted in desperate measures taken, including the decontamination and reuse of face masks.

Research efforts have been invested into finding the best possible way of decontaminating used FFRs for reuse purposes. Several decontamination methods have been shortlisted and even implemented in some hospitals, including the use of ultraviolet (UV) radiation, hydrogen peroxide, ethylene oxide, steam, and heat, which will be discussed in greater details herein. The requirements for an effective decontamination method for masks and FFRs are as follows: (i) pathogens contaminated on the surfaces of masks and FFRs must be effectively killed and inactivated; (ii) there must no reduction in the filtering performance of masks and FFRs towards pathogens and particulates; (iii) structural integrity of all other components of the masks and FFRs (including elastic straps and metallic noseband) must not be adversely affected; (iv) for FFRs, tight-fitting to the users' face must not be compromised; and (v) decontamination must not leave behind chemicals or by-products that may affect the health and well-being of users. In addition, other considerations have to be taken to ensure the decontamination method can be practically carried out in a sufficient scale, by hospitals or individuals at home. These include (i) the availability and cost of resources, including space, equipment, and chemicals; (ii) the ease and robustness of the process; (iii) safety to the person performing the decontamination process, especially when it dues to with harmful radiation or chemical fumes; and (iv) the scale at which the process can be performed. Research efforts in developing and optimising mask decontamination methods therefore commonly involve (i) testing the effectiveness of the methods in killing different pathogens coated over mask surfaces; (ii) testing the filtering performance, fit factor, and structural integrity of masks after decontamination; and (iii) determining how many cycles of decontamination processes can the masks undergo before deteriorations were detected.

Several review articles and websites have effectively summarised research findings and outcomes derived from efforts in this area [96-98]. For instance, the webpage N96DECON provides online resources on methods of decontaminating
N95 masks [99], whereas the CDC of the USA has dedicated a webpage making recommendations on appropriate FFR decontamination methods [3]. In addition, recent research efforts have also been undertaken to test these methods for their effectiveness against the SARS-CoV-2 virus, given many of these methods were reported before the COVID-19 pandemic and tested against a range of different bacteria and viruses. In general, there are many ways and chemical agents (e.g., bleach and soap) that can be used to disinfect pathogens on our hands, small items, and common surfaces of high touchpoint, but not all of these can be practical for mask decontamination.

5.1. Decontamination by UV Radiation. Short wavelength UV radiation (UV-C, $\lambda=254 \mathrm{~nm}$ ) is commonly used to disinfect small items, which can kill pathogens in a matter of minutes of exposure. UV radiation has been commonly adopted to disinfect medical items in hospitals, vehicles in bus and train depots, scissors and combs in barbershops, and even baby milk bottles at home. Ultraviolet germicidal irradiation (UVGI) has therefore been one of the most commonly studied and adopted methods for the decontamination of masks and FFRs. The effectiveness of UVGI in mask decontamination depends on three important factors: (i) the intensity of UV radiation, (ii) the duration of exposure, and (iii) the dimension and direction of UV radiation with respect to the mask. For the first two factors, prolonged exposure to very high-intensity UV radiation may result in the degradation of mask materials, which may compromise the filtering capacity and the mask-fitting (for FFRs). An optimal radiation intensity and treatment duration must therefore be fine-tuned. For the third factor, the even exposure of all possible surfaces of the mask to UV radiation will be ideal for thorough decontamination, but this may be challenging based on how users position both the UV sources and the masks in the disinfection chamber.

To study the effects of UVGI treatments on N95 respirators, Viscusi et al. exposed FFRs, 15 minutes on each side, to $176-181 \mathrm{~mJ} / \mathrm{cm}^{2}$ of UV radiation $[100,101]$. No visible changes were observed to the FFRs, while both the filter aerosol penetration and filter airflow resistance were not affected as well. Bergman et al. evaluated the effects of three 15minute UVGI treatment cycles $\left(1.8 \mathrm{~mW} / \mathrm{cm}^{2}\right)$ on FFRs $[102,103]$. No degrading effects on filtration performance or face-fitting were observed for the different models of FFRs tested as all treated masks managed to pass quality and safety standards. Lindsley et al. reported that there was a small increase in particle filtration performance (up to $1.25 \%$ ) but little effect on flow resistance for most models of FFRs upon undergoing UVGI treatment with an exposure dose between 120 and $950 \mathrm{~J} / \mathrm{m}^{2}$ [104]. The elastic strap, however, experienced decreases in breaking strength as the dosage of UV radiation increases. More recently, Liao et al. reported that face masks can maintain filtration efficiency above $95 \%$ after 10 cycles of UVGI treatment $\left(\sim 3.6 \mathrm{~J} / \mathrm{cm}^{2}\right)$, only to show small degradation to $\sim 93 \%$ after 20 cycles [105].

The decontamination effectiveness of UVGI treatment was studied. Earlier, Fisher and Shaffer investigated the decontamination effects of UV treatment on the different 
layers of masks by exposing different models of N95 FFRs exposed to MS2 coliphage to UV-C radiation at a minimum dose of $1000 \mathrm{~J} / \mathrm{m}^{2}$ for durations of 2 to 266 minutes [106]. The porous nature of N95 FFRs' materials allows UV radiation to penetrate through different layers, thus registering at least a 3-log reduction of viable MS2. Heimbuch et al. demonstrated the effectiveness of UVGI treatment (15-minute exposure at a dose of $18 \mathrm{~kJ} / \mathrm{m}^{2}$ ) on N95 FFRs contaminated with $\mathrm{H} 1 \mathrm{~N} 1$ aerosols and droplets, where an average logreduction of 4.69 and 4.92 was recorded, respectively [107]. In a separate study, Mills et al. reported more than a 3-log reduction in $\mathrm{H} 1 \mathrm{~N} 1$ virus viability in 12 and 7 facepieces and elastic straps, respectively, out of 15 UVGI-treated (1minute exposure at a dose of $1 \mathrm{~J} / \mathrm{cm}^{2}$ ) N95 FFRs of different models [108]. More recently, Fischer et al. and Ou et al. assessed the feasibility of UVGI for decontaminating SARSCoV-2-contaminated masks. The former concluded that UV-irradiation at wavelength $260-285 \mathrm{~nm}$ can effectively sterilize the N95 FFRs up to three cycles with no compromise to mask performance [109], whereas the latter reported the that N95 FFRs and surgical masks may be effectively decontaminated at an exposure dose of $216 \mathrm{~mJ} / \mathrm{cm}^{2}$ for 5 minutes for up to 10 cycles, without significant deterioration of filtration efficiency and fit factor [110].

While UV radiation is harmful to human skin and eyes, UVGI treatment of contaminated masks serves as an attractive decontamination method which avoids the use of toxic chemicals. In a hospital in Nebraska (USA), for example, decontamination was performed by having contaminated masks hanged on wires drawn across an empty room fitted with two UV light towers [111]. A total of 2000 masks can be sterilized a day based on this UVGI treatment method. Nonetheless, one drawback to UVGI treatment is the unlikeliness in achieving homogenous decontamination across the entire exterior surface of the masks and FFRs due to the UV light not being able to reach the "shadowed areas" and crevices attributed to the positioning of masks with respect to the UV source, and multiple pieces of a mask being treated together [96].

5.2. Decontamination by Hydrogen Peroxide Vapour. Hydrogen peroxide $\left(\mathrm{H}_{2} \mathrm{O}_{2}\right)$ solution is a common antiseptic in wound treatment whereas $\mathrm{H}_{2} \mathrm{O}_{2}$ vapour (HPV) is often generated to disinfect enclosed spaces, such as offices, workstations, hospital wards, and interiors of buses, trains, and aircraft, as well as to sterilize laboratory and medical equipment and tools in specially designed enclosed chambers. The generation of HPV by hospitals and cleaning companies for the disinfection of common spaces can be achieved with the use of portable "vaporizer" machines. Likewise, the easy generation of HPV at low temperatures makes it a desirable method for disinfecting masks and FFRs. Unlike UVGI, HPV is able to reach all "shadowed areas" and crevices of the mask exteriors during treatment, thus ensuring decontamination to be more thorough. Likewise, the decontamination effectiveness of vaporized $\mathrm{H}_{2} \mathrm{O}_{2}$ (VHP) treatment and its effect on mask performance and structural integrity depend on exposure time, VHP concentration, and treatment regime, which often involves dehumidification, conditioning, dwell, gassing, and aeration. In addition, $\mathrm{H}_{2} \mathrm{O}_{2}$ readily decomposes into oxygen and water so exposure to residual $\mathrm{H}_{2} \mathrm{O}_{2}$ is not a big issue. The VHP method is used in some hospitals due to the availability of $\mathrm{H}_{2} \mathrm{O}_{2}$ vaporizer machines. For instance, Hospitals in Ohio (USA) under OhioHealth worked with research organization Battelle Memorial Institute to retrofit enclosed rooms in hospitals for VHP decontamination, which can treat thousands of masks in one go [111].

In general, the VHP method is able to effectively decontaminate masks from different bacteria and viruses without compromising mask performance. Early works by Viscusi et al. reported that N95 FFRs undergoing VHP treatment for up to 55 minutes and temperature up to $80^{\circ} \mathrm{C}$ only exhibited slight tarnishing of metallic nosebands without significant changes to filtering capacity $[100,101]$. Bergman et al. further confirmed that N95 FFRs undergoing 3 cycles of VHP treatment with HPV concentration of $8 \mathrm{~g} / \mathrm{m}^{3}$ for 125 minutes (each cycle) do not yield any degradation to filtering performance as well, with recorded mean filter penetration of below $4.01 \%$ [102]. The nondamaging nature of VHP treatment was validated by a laboratory testing conducted by Battelle and funded by FDA (in the USA); N95 FFRs were found to still meet filtering performance and fit requirement even after 50 cycles of VHP treatment using a Bioquell Clarus C HPV generator, although elastic straps started to deteriorate [112]. More recently, Schwartz et al. reported similar findings through the decontamination of $1003 \mathrm{M}^{\mathrm{TM}}$ N95 FFRs via treatment with $480 \mathrm{ppm}$ of HPV, involving five stages: conditioning, pregassing, gassing (25 minutes), gassing dwell (20 minutes), and aeration, with no physical or performance degradation recorded [113].

In view of the COVID-19 pandemic, the suitability of VHP for the treatment of SARS-CoV-2-contaminated masks was studied. Kenney et al. demonstrated the effectiveness of VHP treatment on N95 FFRs, which demonstrated a complete eradication of 3 aerosolized phages (that mimicks the SARS-CoV-2): T1, T7, and Pseudomonas phage phi-6 in just one cycle of treatment, involving 10 minutes of conditioning, $30-40$ minutes of gassing at $16 \mathrm{~g} / \mathrm{m}^{3}$ of HPV, 25 minutes of dwelling, and 150 minutes of aeration [114]. No deformity was observed for the FFRs after 5 cycles of treatment. Similarly, Kumar et al. reported no recoverable SARS-CoV-2 viruses on N95 FFR surfaces after the masks undergo a one-hour treatment cycle involving 10 minutes of dehumidification, 3 minutes of conditioning, 30 minutes of decontamination, and 20 minutes of aeration. Throughout the process, the peak HPV concentration of $750 \mathrm{ppm}$ and the FFRs can undergo 10 cycles of treatment without compromise to mask performance [115]. Likewise, Smith et al. also reported no functional degradation to N95 FFRs (both filtering capacity and fit factor) after two cycles of VHP treatments involving 20 minutes of gassing ( $500 \mathrm{ppm}), 60$ minutes of dwelling $(\sim 420 \mathrm{ppm})$, and 210 minutes of aeration at ambient temperature, with no viable virus detected at the end of each cycle [116]. Finally, Fischer et al. concluded that among VHP, UVGI, heat, and ethanol treatment, VHP treatment displayed the best combination of rapid inactivation of SARSCoV-2 and preservation of N95 FFR integrity [109]. 
5.3. Decontamination by Ethylene Oxide Vapours. There has been some early research work conducted on the viability of using ethylene oxide vapour to decontaminate FFRs. Viscusi et al. reported that treating FFRs with ethylene oxide vapour $(725-883 \mathrm{mg} / \mathrm{L})$ at $55^{\circ} \mathrm{C}$ for 1 hour, followed by 4 hours of aeration, do not yield any visible sign of mask degradation, with the exception of darkening of elastic straps $[100,101]$. The treated masks passed filtration performance assessments. More recently, Kumar et al. reported that N95 FFRs undergoing $1 \mathrm{hr}$ exposure to ethylene oxide vapour followed by 12 hours of aeration managed to achieve complete sterilization of SARS-CoV-2 viruses [115]. The masks can tolerate at least 3 cycles of treatment without any significant structural or functional deterioration. However, there were concerns about the toxicity of ethylene oxide towards mask wearers as it is potentially carcinogenic and teratogenic. In addition, ethylene oxide is flammable, which posed fire safety hazards to the treatment process as well. CDC does not recommend the decontamination of masks using this method [3].

5.4. Decontamination by Heat, Moist, and Steam. Heat, moist, and steam appear to be one of the most popular ways of decontamination because they do not require the use of dangerous chemicals and radiation or any sophisticated equipment. The simplicity of such treatments can even be replicated or performed at home with the use of microwave ovens, rice cookers, and steamers. For the use of microwave ovens, steam treatment may be carried out in a microwave steam bag, which is not an uncommon household item, given its uses in cooking or disinfecting items used for babies. The downside to this is method is that given the size of microwave ovens, the treatment method cannot be performed one mask at a time. There were also concerns that prolonged exposure to microwave radiation may cause deterioration of mask performance. On the other hand, people in Taiwan were advised to decontaminate their used surgical masks via "dry steaming" in a rice cooker for 3 minutes, which was said to achieve 99.7\% sterilization rate although the mask's filter quality would deteriorate by $10 \%$ [117]. The effectiveness of decontamination via moist heat treatment varies according to temperature and relative humidity (RH). Exposing masks and FFRs to too high heat may compromise structural integrity and performance. There were also debates over the importance of moisture and humidity in the sterilization process. Li et al., for instance, demonstrated that Staphylococcus aureus and MS2 phage inoculated masks experienced $<3$ and $>5$ log-reductions in both pathogens upon undergoing 15 minutes of dry heating and steam treatment, respectively, thus reflecting the importance of humidity in decontamination [118].

Earlier, Viscusi et al. reported N95 FFRs that underwent dry heating in an oven at $80^{\circ} \mathrm{C}$ for 60 minutes do not yield any visible physical changes whereas heating at $160^{\circ} \mathrm{C}$ for 22 minutes caused the polypropylene masks to melt and become unusable [101]. On the other hand, partial melting and increased filter penetration were observed for FFRs that underwent 2 minutes of dry microwave treatment $\left(750 \mathrm{~W} / \mathrm{ft}^{3}, 1\right.$ minute per side) [100]. Bergman investigated the effects of 3 cycles of mask decontamination treatments via microwave oven generated steam (MGS, 2 minutes each cycle) and moist heat incubation (MHI, 15-30 minutes each cycle, $\left.60^{\circ} \mathrm{C}, 80 \% \mathrm{RH}\right)$. MGS and MHI treatments were both found to cause partial separation of the inner foam nose cushion for some of the FFRs, whereas slight melting of the head straps was also observed for MGS-treated FFRs due to sparking in the microwave $[102,103]$. More recently, Liao et al. concluded that decontaminating face masks by heating below $85^{\circ} \mathrm{C}$ under various humidity levels appears to be a promising, nondestructive method for the preservation of filtration properties in melt-blown fabrics as well as N95 FFRs [105]. Face masks treated at $85^{\circ} \mathrm{C}$ at $30 \% \mathrm{RH}$ can undergo 50 treatment cycles without significant changes in the filtration efficiency. Recent studies from Ou et al. also concluded that filtration efficiency and fit factor of N95 FFRs were not significantly compromised after 10 repeated cycles of both 30minute steam treatment as well as 30 -minute oven-dry heating at $77^{\circ} \mathrm{C}[110]$.

Investigating the effectiveness of different sterilization methods, Heimbuch et al. reported that no $\mathrm{H} 1 \mathrm{~N} 1$ viruses survived warm moist heat $(\mathrm{WMH})$ treatment $\left(3 \mathrm{hr}, 65^{\circ} \mathrm{C}, 85 \%\right.$ $\mathrm{RH}$ ), whereas sporadic viable viruses were detected after MGS (with a water reservoir, 2 minutes, $1250 \mathrm{~W}$ ) due to homogenous delivery of warm moisture by the former versus nonuniversal distribution of steam by the latter [107]. Likewise, Fisher et al. reported more than 4 log-reduction in MS2 phage viability with just 45 seconds of MGS treatment [119]. Fisher et al. further evaluated the viability of using microwave steam bags to perform MGS decontamination of FFRs, where the method yielded $99.9 \%$ efficiency in killing MS2 phage with a filtration efficiency of posttreated FFRs remaining above 95\% [120]. More recently, $\mathrm{Ma}$ et al. subjected face masks made of melt-blown polypropylene to steam treatments of different durations (20-120 minutes) and found that they were still able to effectively block 98 to 99\% of aerosolized H120 virus [121]. Likewise, Xiang et al. reported that dry-heating contaminated surgical face masks and N95 FFRs at 60 and $70^{\circ} \mathrm{C}$ for an hour can effectively kill different bacteria and fungi pathogens, as well as inactivating $\mathrm{H} 1 \mathrm{~N} 1$ virus. In addition, no physical changes were observed for both surgical face masks and N95 FFR even after heating for 3 hours at $70^{\circ} \mathrm{C}$, where their filtering capacity remains at 97 and 96\%, respectively [122]. Similarly, Fischer et al. reported that SARS-CoV-2-contaminated N95 FFRs that underwent dry-heating at $70^{\circ} \mathrm{C}$ can achieve effective sterilization with negligible changes to mask fit factor, even after 2 cycles of treatment [109].

5.5. Decontamination by Disinfectant Solution Treatments. Treatment of contaminated face masks by soaking in disinfectant solutions may not be the most preferred way of mask decontamination. One reason is because it involves posttreatment drying of a mask which may not only take a long time but also cannot guarantee the complete removal of residual disinfectant chemicals, which may be unpleasant to smell and/or pose health hazards. In addition, many common disinfectant solutions may be detrimental to the mask's structural integrity, thus compromising its filtering performance 
and fit factor after treatment. Choosing the right disinfectant solution is therefore important to ensure that posttreated masks not only do not degrade in structure and performance but also ensure disinfectant can be thoroughly removed to avoid odour and health threats to mask users.

Early studies by Viscusi et al. and Bergman et al. suggest that treatments in liquid $\mathrm{H}_{2} \mathrm{O}_{2}$ were not found to affect the filtering performance of N95 FFRs [101, 102]. FFRs submerged in $3 \% \mathrm{H}_{2} \mathrm{O}_{2}$ for 30 minutes were reported to not yield significant visual changes, but those submerged in $6 \% \mathrm{H}_{2} \mathrm{O}_{2}$ experienced ink fading on the exterior [101]. Three cycles of treatments in $6 \% \mathrm{H}_{2} \mathrm{O}_{2}$ were also reported to cause staples on the FFRs to oxidise to varying degrees [102]. The effectiveness of decontamination by liquid $\mathrm{H}_{2} \mathrm{O}_{2}$ was however not evaluated.

Bleach was found to be a less desirable decontaminating agent due to the issue of odour. Viscusi et al. reported an increase in average filter aerosol penetration in different models of FFRs soaked in 0.525, 5.25\% bleach solutions, although penetration of N95 FFRs still remains within 5\% threshold [100, 101]. Filter airflow resistance of N95 FFRs was not affected much as well after 30 minutes of soaking in $6 \%$ bleach [100]. Also, the strong smell of bleach can still be detected even after the overnight drying of the treated masks [100]. Similar findings were made by Bergman et al. for FFRs after undergoing 3 cycles of bleach treatment (30 $\mathrm{min}, 0.6 \%$ ) [102].

Ethanol was reported unsuitable for use for mask decontamination. The soaking of contaminated masks in 70\% ethanol was recently reported by Smith et al. to cause impairment in mask function within 30 minutes although no SARS-CoV-2 viral RNA could be cultured [116]. Similarly, rapid inactivation of SARS-CoV-2 by $70 \%$ ethanol treatment was also reported by Fischer et al. but a loss of structural integrity of the N95 FFRs was resulted [109].

Although handwashing with soap can effectively kill pathogens, treating contaminated FFRs with soap water may not be a practical idea. Viscusi et al. found that although no visible changes were observed for FFRs soaked in soap water for 2 and 20 minutes, average penetration was found to increase significantly [101]. This was attributed to the surfactant of soap removing charges on electret fibres of the filtering material that plays an important role in preventing aerosol and particulate penetration.

5.6. Decontamination by Other Methods. Other disinfection methods were studied for decontaminating used masks including the use of autoclaves and disinfectant wipes. Autoclave treatment is commonly used to sterilize medical tools and apparatus via pressurized saturated steam at $121^{\circ} \mathrm{C}$. Viscusi et al. reported the increase in average penetration, as well as deformation, shrinking, stiffening, and mottling of N95 FFRs after 15 and 30 minutes of autoclave treatment [100, 101]. On the contrary, a recent study by Kumar et al. reported that masks with layered fabric, pleated models tolerated 10 cycles of autoclave treatment while maintaining structural and functional integrity, whereas masks with more rigid moulded models demonstrated loss of function after one autoclave cycle [115].
Disinfectant wipes are convenient to use but their effectiveness in decontaminating masks may not be ideal. Heimbuch et al. studied the effectiveness of 3 different wipes, containing benzalkonium chloride (BAC), $0.9 \%$ hypochlorite (OCL), and no active antimicrobial ingredients (inert), respectively, on mask decontamination [123]. After $30 \mathrm{sec}-$ onds of wiping, it was found that BAC- and OCL-wipe can only achieve 3 to 5 log-reduction in pathogens whereas inert wipe can only achieve $\sim 1$ log-reduction. Although average penetration remains below $5 \%$ after the use of all three wipes, there was an issue of uneven surface cleaning across different components of the mask [123].

Surgical masks and FFRs are made to be used only once and not meant to be reused. Nonetheless, should the need of reuse arise due to the issue of shortage, several wellstudied decontamination methods can be considered. Among them, UVGI, VHP, and moist heat treatment appear to be more effective and suitable for large scale decontamination of mask in the hospital setting, whereas moist heat, dry heat, and steam treatment can be performed easily at home. Some other methods may not be practical as they may result in the compromise of structural integrity and degradation of performance. In fact, mask producer $3 \mathrm{M}$ has issued online technical bulletins to advise on the suitability of different models of FFRs for different methods of decontamination treatments [124]. Even more important, there is a need for proper handling of contaminated masks in the process of preparing for decontamination. Cautions must be practiced to avoid physical contact with a contaminated surface or exposure to viral-loaded aerosols that may be generated in the process. It is therefore advisable to wear protective gloves, masks, and even goggles when performing mask decontamination and to wash hands thoroughly with soap or disinfectant after that.

\section{Engineering of Multifunctional Masks and Mask Materials}

While existing models of masks and respirators serve users well in terms of the level of protection they offer against airborne pathogens, there has still been intensive research and developmental efforts to improve their filtering properties and performances, as well as comfort and userfriendliness. Such efforts can be categorised into two aspects: (i) improving the filtering capacity of mask material and (ii) engineering additional functions and properties into the designs of masks. The former involves material development and engineering-how do we process bulk materials to reduce their pore sizes such that they are sufficiently small to capture and filter off minute particulates and pathogens, and how do we treat or develop these materials to enable them to inactivate microorganisms. The latter involves making changes to the existing design of mask models-to confer on their antimicrobial properties through the application of coatings, for instance, and improve user comfort, friendliness, and convenience such as the introduction of sensing and self-cleaning properties. 
6.1. Improving Air Filter Performance for Particulate Matter Capture. High-performance air filters should be able to capture particulate matter (PM) tiny particles with high efficiency while maintaining air permeability. Currently, the existing commercial air filters for PM capture are mainly composed of a mat of randomly arranged polymer fibres or fibreglass with diameters ranging from several microns to tens of microns. These filters always function by passively trapping PM which highly depends on the porous structure of the fibrous membrane. In order to achieve high removal efficiency, thick layers of densely packed fibres are needed and air permeability is therefore sacrificed. Recently, a variety of novel membrane filters have been developed for PM purification with enhanced performance and attractive characteristics such as smaller fibre diameters, higher specific surface area, low air resistance, and transparency, while being light and having functionalized active surfaces to capture particles more efficiently by electrostatic forces or chemical bond interactions. In this section, we will introduce the most popular innovative air filtration materials developed in the past five years for PM capture, including polymer nanofibre membranes, electret membranes, and porous metal-organic framework- (MOF-) based filters.

6.1.1. Polymer Nanofibrous Membranes. The key factors affecting the functions of air filters are fibre diameter, membrane thickness, and air permeability. When the fibre diameter is decreased to nanoscale, the PM removal efficiency can be greatly improved due to enhanced specific surface area and high porosity, and thus, the membrane thickness can be reduced to ensure low air resistance. Among all the methods for fabricating nanofibrous membranes, electrospinning is the most widely used versatile technique which produces continuous nanofibres through an electrically charged jet of polymer solution [125]. Owing to the large specific surface area and highly interconnected porous network, electrospun membranes have been extensively employed for water treatment, biomedical and energy-related applications over the past decades, and they have recently attracted renewed research interest since Lui's group demonstrated the high efficiency of electrospun fibres for air purification [126]. A transparent polyacrylonitrile (PAN) nanofibre membrane with an average fibre diameter of $\sim 200 \mathrm{~nm}$ was fabricated by electrospinning and evaluated for the capture of $\mathrm{PM}_{2.5}$ particles. Compared with existing commercial air filters made of thick layers of micron-sized fibres which balance air resistance and filtering performance, the obtained nanofibre membranes displayed good optical transparency (up to $90 \%$ ), high filtration efficiency (>95\%), low-pressure drop (down to $132 \mathrm{~Pa}$ ), and light weight. Strong adhesion of PM to the PAN nanofibre surface was demonstrated by the observation of in situ PM capture process. The developed transparent thin filter can be applied to indoor air protection through windows or incorporated into existing personal masks. Inspired by Lui's promising results, a variety of electrospun nanofibre membranes with different surface chemistry and mechanical or thermal properties have been developed from polymers, polymer blends, or polymer composites with surface-functionalized inorganic nanofillers for air purification investigations, including polyurethane [127], polycarbonate [128], poly(vinyl alcohol) [129], polytetrafluoroethylene [130], polybenzimidazole [131], polyacrylonitrile/polysulfone [132], polypropylene/polyethylene [133], polyurethane/polysulfonamide [134], polyacrylonitrile/graphene oxide [135], and polyacrylonitrile/MXene [136]. Besides conventional electrospinning, polymer nanofibre membranes mass-produced by needless electrospinning [137] and solution blow spinning [138] also demonstrated effectiveness for the capture of particulate pollutants.

To further improve capture efficiency towards ultrafine particles and reduce weight and packing density of the filters to ensure low resistance to airflow, polymer membranes with a novel nanofibre/net hierarchical porous structure have recently been developed using the cutting-edge electrospinning/netting technology, which is a versatile one-step process for fabrication of polymer membranes comprising common electrospun nanofibres interconnected with twodimensional nanonets [143]. The nanonets, which are formed from the small charged droplets other than electrospinning jets under high electric field, exhibit amazing characteristics such as ultrafine diameter $(<20 \mathrm{~nm})$, high porosity, small pore sizes $(<200 \mathrm{~nm})$, and large specific surface area, making them attractive candidates for fine particulate filtration. In 2015, Wang's group fabricated ultralight nanofibrenets binary nylon 6-PAN with high coverage $(>98 \%)$ of nylon nanonets and low packing density of PAN nanofibres (Figure 10(a)) [139]. The prepared interconnected membranes displayed high filtration efficiency (99.99\%) towards $300 \mathrm{~nm}$ aerosol particles with a low basis weight of $2.94 \mathrm{~g} \mathrm{~m}^{-}$ 2 and satisfactory quality factor $\left(0.1163 \mathrm{~Pa}^{-1}\right)$ under a high flow rate $\left(90 \mathrm{~L} \mathrm{~min}^{-1}\right)$, which is significantly superior to that of commercial glass fibre and melt-blown polypropylene fibre-based filtration membranes. Later, the same group further developed a highly integrated multilayer air filter comprising polysulfone microfibre (diameter $\sim 1 \mu \mathrm{m}$ ), polyacrylonitrile nanofibre (diameter $\sim 200 \mathrm{~nm}$ ), and polyamide- 6 nanonets (diameter $\sim 20 \mathrm{~nm}$ ) via sequential electrospinning [144]. The integrated filter with gradually varied pore structures and high porosity can efficiently capture airborne particles in a gradient manner with low air resistance. Very recently, Li et al. also employed the electrospinning/netting process for the mass production of a reusable poly(vinylidene fluoride) nanofibre/nanonet air filter, which presented a high purification efficiency of $99.985 \%$ towards $\mathrm{PM}_{0.26}$ and a low-pressure drop of 66.7 $\mathrm{Pa}$ [145].

6.1.2. Electret Membranes. Unlike the passive membranes that capture particles mainly by the porous structure, the electrostatic air filters are able to effectively trap particles in an active manner with a larger attraction distance. Without depending on the high density of small pores, the filter thickness can be reduced and the removal efficiency can be maintained under a continuous airflow with a low-pressure drop. Three charging techniques, namely, in situ charging, corona charging, and tribocharging, can be used to fabricate electret membranes [146].

The electrospinning process for the fabrication of nanofibrous membranes can in situ charge the nanofibres through 


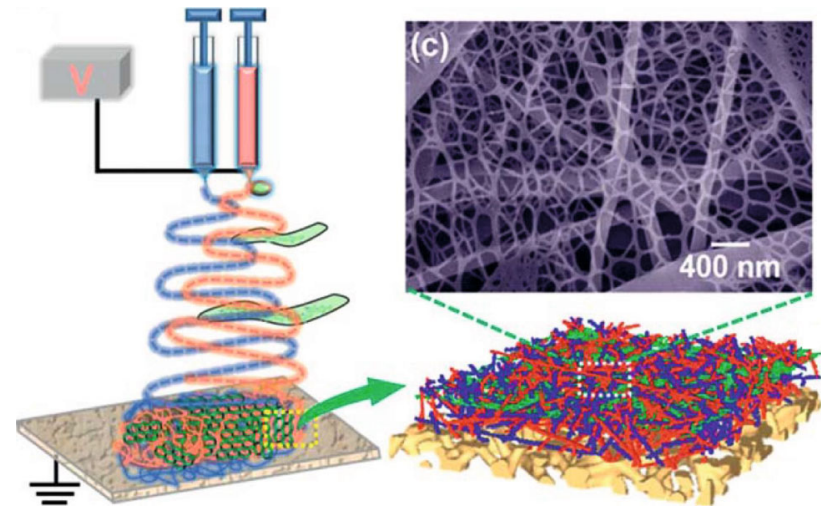

(a)

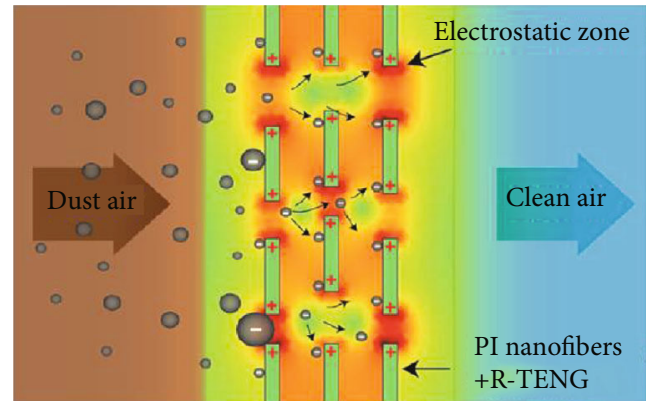

(c)

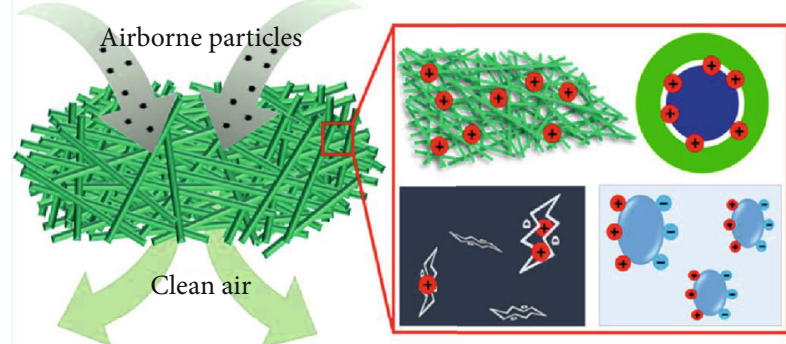

Charges stored in materials (b)

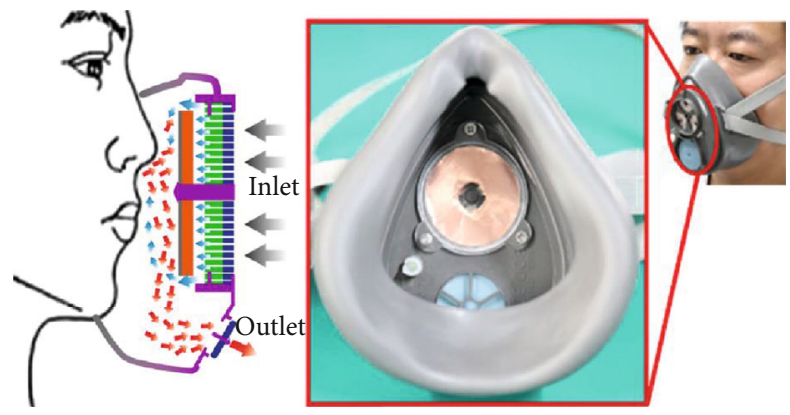

(d)

FIGURE 10: (a) Schematic showing the fabrication of nylon-6/PAN nanofibre/net membranes by electrospinning/netting technology. (b) Schematic showing an electret filter consists of PE/PP nanofibre as the matrix and magnesium stearate as the charge enhancer. (c) Schematic image of the filtration mechanism of the filter with R-TENG. (d) Structure and photo of a self-powered electrostatic adsorption face mask with R-TENG. Reprinted with permission from Ref. [126], copyright 2015, Macmillan Publishers Limited; Ref. [139], copyright 2015, The Royal Society of Chemistry; and Ref. [140-142], copyright 2017-2019, American Chemical Society.

introducing charge storage enhancers into electrospinning solutions. Nanoparticles, such as polytetrafluoroethylene, silicon nitride, magnesium stearate, titanium dioxide, boehmite, and $\mathrm{SiO}_{2}$, are usually employed as charge enhancers, and various hybrid electret filters have been developed via the in situ charging technology of electrospinning [140, 147-149]. An electrospun polyethylene/polypropylene bicomponent membrane containing magnesium stearate (Figure 10(b)) was endowed with the elevated surface potential of $4.78 \mathrm{kV}$ and exhibited a high filtration efficiency of 98.94\% towards $\mathrm{PM}_{2.5}$ with a low-pressure drop of $37.92 \mathrm{~Pa}$ and excellent dust holding capacity of $10.87 \mathrm{~g} \mathrm{~m}^{-2}$ [140]. A polyvinylidene fluoride nanofibrous membrane doped with well-dispersed $\mathrm{SiO}_{2}$ nanoparticles demonstrated a remarkable electret effect with a surface potential of $12.4 \mathrm{kV}$ and high filtration performance towards particles with different sizes [149]. Besides electrospinning, corona treatment is another approach to charge fibrous membranes under an external electric field. Zhang et al. fabricated electret polypropylene nonwovens via melt blowing followed by corona charging with magnesium stearate as the charge enhancer [150]. After being charged at a voltage of $100 \mathrm{kV}$ for $30 \mathrm{~s}$, the electrostatic nonwoven filter demonstrated high filtration efficiency up to $99.22 \%$ against $\mathrm{PM}_{2.5}$, low-pressure drop of $92 \mathrm{~Pa}$, and satisfactory QF value of $0.054 \mathrm{~Pa}^{-1}$.

A fatal drawback of the in situ and corona-charged electret membranes is the poor stability of filtration efficiency due to the rapid dissipation of the surface charges when the membranes are in contact with moisture or oil droplets under a hazy environment [151]. Electret membranes fabricated by a tribocharging strategy with constant charge supply can solve the problem of charge dissipating, leading to enhanced stability and prolonged service life of the filters. Very recently, a triboelectric nanogenerator (TENG) has been combined with nanofibrous air filters for highefficiency particulate removal [141, 142]. TENG is a newly invented technology which is used for harvesting energy from various mechanical movements such as wind, water wave, and human motion [152]. The large open-circuit voltage (up to several hundred volts) generated by TENG based on triboelectrification and electrostatic induction effect makes it a popular candidate for application in various selfpowered wearable devices [153]. In 2017, Gu's group first invented a rotating triboelectric nanogenerator (R-TENG) enhanced electrospun polyimide membrane for air purification. The polyimide nanofibre membrane was positively charged by R-TENG in a constant manner, leading to greatly improved removal efficiency towards PM particles with diameters less than $100 \mathrm{~nm}$ (Figure 10(c)) [141]. Using this technology, the same group later developed a self-powered electrostatic adsorption face mask (SEA-FM) from electrospun poly(vinylidene fluoride) membrane equipped with a TENG driven by human respiration (Figure 10(d)) [142]. The SEA-FM exhibited removal efficiency higher than 


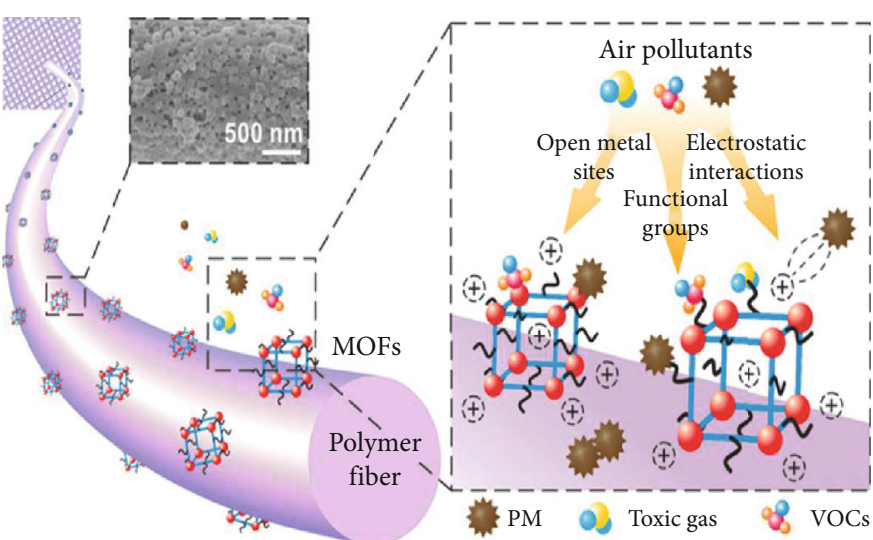

(a)

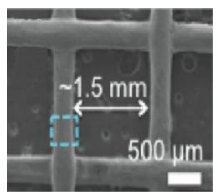

(c)

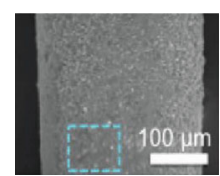

(d)

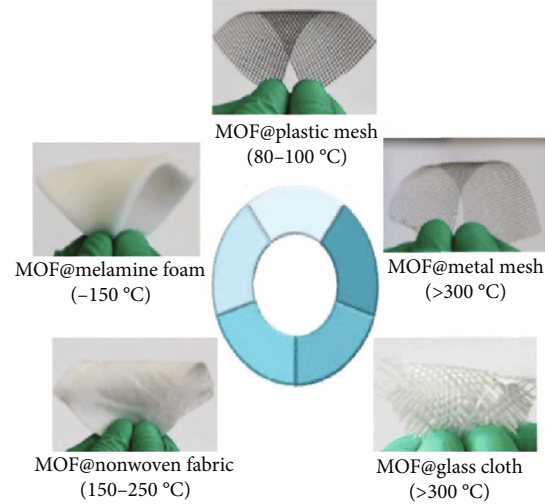

(b)

FIgure 11: (a) Proposed capture mechanism of the MOF-based filter for air pollutants. Inset is the SEM image of the surface of the MOF/polymer composite fibre. (b) MOF filters based on various flexible substrates produced by a roll-to-roll hot-pressing method. (c-e) SEM images of the ZIF-8@plastic mesh achieved after seven layer-by-layer coating cycles. Reprinted with permission from Ref. [159] and Ref. [156]. Copyright 2016 and 2017, the American Chemical Society and WILEY-VCH.

$99.2 \mathrm{wt} \%$ towards coarse and fine particulates with a lowpressure drop, and the efficiency towards ultrafine particulates was still as high as $86.9 \mathrm{wt} \%$ after continually working for $240 \mathrm{~min}$ and a 30 -day interval. Very recently, Bai's group presented a washable and reusable multilayer triboelectric air filter which consists of multilayers of nylon and polytetrafluoroethylene fabrics, which can be easily charged through rubbing against each other [154]. A high open-circuit voltage of $190 \mathrm{~V}$ can be generated on the surface of the fabrics, leading to a high removal efficiency of $84.7 \%$ for $\mathrm{PM}_{0.5}$ and $96.0 \%$ for $\mathrm{PM}_{2.5}$. The removal efficiency is stable under a high humidity environment and displayed no obvious deterioration after five cleaning cycles. Hence, the triboelectric air filters are highly effective and stable with a long service life, paying the way for the fabrication of face masks and other health protection applications.

6.1.3. MOF-Based Filters. Metal-organic frameworks (MOFs) are a class of porous crystalline materials that are composed of transition-metal cations and coordinately bonded multidentate organic linkers. With high porosity, tunable pore size, rich functionalities, and good thermal stability, MOFs hold great promise for applications as filtration materials [155]. As MOF crystals are in a light powder form, they are usually grown on porous substrates or embedded in polymer fibrous membranes to form MOF-based filters. Li's group first explored the interactions between MOFs and particulate pollutants via the incorporation of ZIF-8 nanocrystals in electrospun PAN membranes [135]. It was proposed that the particulate pollutants can be captured by the MOFbased filters via three mechanisms: (i) binding to the open metal sites on MOFs, (ii) interacting with the functional groups on MOFs and/or polymers, and (iii) electrostatic interactions with MOF nanocrystals (Figure 11(a)). Due to the unbalanced metal ions and defects on the surface, MOFs can offer positive charge to polarize the PM surface, leading to improved electrostatic adsorption of PM pollutants. The specific surface area of the PAN filter was dramatically improved from 115 to $1024 \mathrm{~m}^{2} \mathrm{~g}^{-1}$ after incorporation of 60 wt\% ZIF-8 nanocrystals. The developed ZIF-8@PAN filters exhibited high PM removal efficiencies up to $88.33 \%$ and $89.67 \%$ for $\mathrm{PM}_{2.5}$ and $\mathrm{PM}_{10}$, respectively, with an ultralow pressure drop of less than $20 \mathrm{~Pa}$. In addition to PM pollutants, these MOF filters were also found effective in selective capture of $\mathrm{SO}_{2}$ when exposed to a stream of $\mathrm{SO}_{2} / \mathrm{N}_{2}$ mixture. Inspired by the promising results, the same group further employed a roll-to-roll hot-pressing method for mass production of MOF-based filters on various commercially available flexible substrates (i.e., plastic mesh, glass cloth, metal mesh, nonwoven fabric, and melamine foam) (Figure 11(b)) [156]. The produced MOF filters demonstrated excellent performance for PM removal under a wide range of working temperatures from 80 to $300^{\circ} \mathrm{C}$. The PM removal efficiency of the ZIF-8@plastic mesh (Figures $11(\mathrm{c})-11(\mathrm{e})$ ) was retained $>90 \%$ after 30 consecutive days. It can be easily washed with tap water and ethanol and reused three times without apparent efficiency loss, which is quite promising for application in residential pollution control. Following Wang's work, Feng et al. designed a hierarchical, multifunctional UiO-66- $\mathrm{NH}_{2}$ wrapped CNTs/PTFE filter with a high capture efficiency (99.997\%) for ultrafine dust (diameter $\sim 0.3 \mu \mathrm{m}$ ) and $\mathrm{SO}_{2}$ adsorption capacity in dynamic filtration [14]. Koo et al. reported the growth of flowerlike hierarchical 2D assembled MOF on polypropylene 
microfibres as a washable membrane filter with high PM removal performance $\left(92.5 \%\right.$ for $\mathrm{PM}_{2.5}$ and $99.5 \%$ for $\left.\mathrm{PM}_{10}\right)$, low-pressure drop $\left(10.5 \mathrm{~Pa}\right.$ at $\left.25 \mathrm{~L} \mathrm{~min}^{-1}\right)$, and superior stability after reuse for 12 cycles [157]. Hao et al. developed electrospun polyimide/ZIF-8 nanofibrous membranes with superior thermal stability (up to $300^{\circ} \mathrm{C}$ ), good transmittance, and excellent mechanical properties for efficient $\mathrm{PM}_{2.5}$ capture (up to $96.6 \%$ with a $10 \mathrm{wt} \%$ ZIF loading), which can be used in harsh conditions such as car exhaust filtration [158].

6.2. Improving Filters and Membrane Materials for Microorganism Removal. Though air filters discussed above show excellent capture efficiency for particular matters (PM), microorganisms (or bioaerosols), such as bacteria, viruses, and fungi in the air, adhere to the filter surface, remain viable, and may reproduce within the filter media, which pose a risk of second airborne contamination. Meanwhile, the accumulation of microorganisms in the filter also blocks the filter, leading to reduced ventilation volume and deterioration of the filter [160-162]. Thus, it is highly desirable to develop air filters with antimicrobial properties, especially when the filters are used for respiratory protection, such as masks, and for indoor air purification. Up to now, a wide range of antimicrobial agents, such as natural products, nanoparticles of metal and metal oxide, metal-organic frameworks (MOFs), graphene, and its derivatives, have been investigated to impart air filters with biocidal properties.

6.2.1. Natural Product Extracts. Antimicrobial extracts of natural products have been widely studied as antimicrobial agents for air filters owing to their high antimicrobial activity, low toxicity, low-cost, and gentleness to the environment [163-165]. The microbial toxicity of natural product extracts is generally accredited to the flavonoids they may contain, which kill microbes via the damage of cell membrane function and inhibition of DNA gyrase [160, 162]. Herbal extracts, such as tea tree oils [166], extract of olive [161], extract of Euscaphis japonica [164], grapefruit seed extract [165], mangosteen extracts [167], and especially Sophora flavescens [168-170], have been sprayed on the surfaces of fibrous polymeric filter for antimicrobial properties, and the herbal extract-coated filter demonstrated good antimicrobial activity. Recently, Sim et al. reported activated carbon (ACF) fibre filters deposited by Sophora flavescens, and the ascoated ACF filter exhibited antimicrobial efficiency higher than $90 \%$, with the toluene removing capacity maintained [171]. However, the high antimicrobial activity of these surface-coated filters is often delivered at a high loading of antimicrobial herbal extracts, which would lead to much increased pressure drop $[164,171,172]$. To solve this problem, Choi et al. mixed Sophora flavescens with polyvinylpyrrolidone (PVP) solution for electrospinning and thus prepared antimicrobial nanofibrous membrane. Owing to the uniform dispersion of antimicrobial ingredient across the polymeric nanofibres, the fabricated hybrid nanofibrous filter exhibited excellent filtration efficiency (99.99\%) and superior antimicrobial activity (99.98\%) against Staphylococ- cus epidermidis (S. epidermidis), with a low-pressure drop of $3.9 \%$ compared with the control at a face air velocity of $1.79 \mathrm{~cm} / \mathrm{s}$ [173]. However, the durability of natural product extracts is still a concern, when it comes to a real application as the antibacterial activity may be affected by temperature, or degraded due to a natural oxidation process $[160,168$, 170]. This can be complemented by nonnatural antimicrobial substances.

6.2.2. Nanoparticles of Metals and Their Compounds. Apart from natural herbal extracts, metals and their compounds have also been extensively studied for their antimicrobial application. Nanoparticles of metal and their compounds have garnered huge attention as a potent antimicrobial agent due to their high surface-to-volume ratios compared with their bulky counterparts $[174,175]$. Though each of them has a different mechanism of biocidal action, a generally proposed mechanism includes (i) the disruption of cell membrane metabolism due to the penetration of nanoparticles and/or release of metal ions and (ii) the effect of photocatalytic, as reactive oxygen species (ROS) like hydroxyl (HO) and superoxide radicals $\left(\mathrm{O}_{2}^{-{ }^{-}}\right)$are generated, which induce oxidative stress to microorganisms and cause the ultimate inactivation [174-177].

Nanoparticles of silver (Ag) [178-180], silver compounds $\left(\mathrm{Ag}^{+}\right)$[181], titanium dioxide $\left(\mathrm{TiO}_{2}\right)$ [182, 183], zinc oxide ( $\mathrm{ZnO})$ [184-186], and aluminum and aluminum oxide $\left(\mathrm{Al}_{2} \mathrm{O}_{3}\right)$ [187] have been incorporated to various filters for antimicrobial properties. A synergistic antimicrobial performance is also revealed via their combination with other biocidal agents, such as carbon nanotubes [178, 180, 181]. Apart from antimicrobial air filters, multifunctional air filters, which simultaneously remove PM, microorganisms, and volatile organic compounds (VOCs), have drawn increasing research attention recently $[188,189]$. The integrated multifunctional air filter provides a promising solution to address the high-pressure drop often caused by multiple filters with different functions used in current air filters. Feng et al. designed and fabricated hierarchical $\mathrm{Ag} / \mathrm{ZnO}$ nanorodwrapped PTFE nanofibrous membrane with an excellent dynamic antibacterial property of $\sim 100 \%$ against Escherichia coli (E. coli), and a formaldehyde degradation rate of $60 \%$, with slightly increased gas penetration, taking advantages of the antimicrobial properties and photocatalytic abilities of both $\mathrm{ZnO}$ and nanosilver [188]. In another study, Zhao et al. reported a multifunctional Ag@MWCNTs $\mathrm{Al}_{2} \mathrm{O}_{3}$ hybrid filter, where Ag@MWCNTs with a hierarchical network-like structure uniformly distributed around the pores of the $\mathrm{Al}_{2} \mathrm{O}_{3}$ filter [189]. The antimicrobial functionality of Ag nanoparticles, as well as their catalytic performance for formaldehyde degradation, were greatly enhanced when loaded on high surface area of CNTs. Owing to the synergistic integration, the Ag@MWCNTs $/ \mathrm{Al}_{2} \mathrm{O}_{3}$ hybrid filter demonstrated excellent antimicrobial rate (>98\%) against common indoor microorganisms, outstanding degradation of formaldehyde $\left(99.99 \%\right.$ at $55^{\circ} \mathrm{C}$, and $82.24 \%$ at room temperature), and complete retention for particles with sizes $\leq$ $0.3 \mu \mathrm{m}$ with a pressure drop of $35.60 \%$ compared with the pristine $\mathrm{Al}_{2} \mathrm{O}_{3}$ filter. 


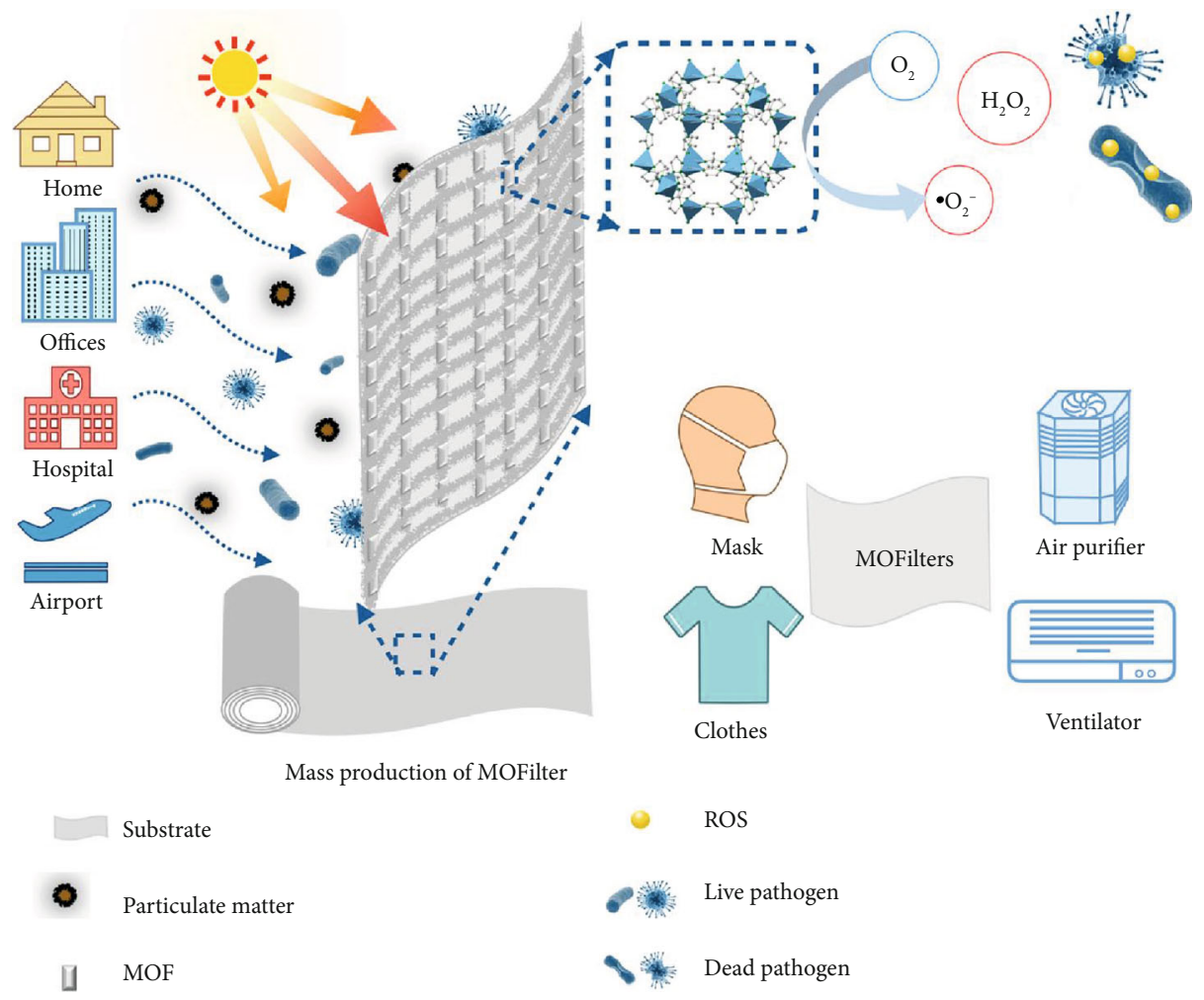

FIGURE 12: Schematic representation of MOF-based filter (MOFilter) for integrated air cleaning. Reproduced with permission from Ref. [196]. Copyright 2019, Springer Nature.

Copper nanoparticles are strong microbicides for a broad spectrum of microorganisms [190]. Very recently, they have been demonstrated to be effective against the newly emerged 2019 coronavirus (COVID-19), which is threatening the whole world $[175,190]$. Though copper-polymer nanocomposites have been explored for antimicrobial applications, the integration of copper nanoparticles to filters for personal protection or air filtration has not been reported yet. With appropriate technologies to effectively deposit copper nanoparticles onto fibrous filter matrix, more advancement for copper nanoparticles as antimicrobial coating in air filters is expected.

6.2.3. Metal-Organic Frameworks (MOF). MOFs, as an emerging new class of antimicrobials, are superior compared with metals due to their high surface area, uniform distribution of metal active sites, and adjustable porous structures $[191,192]$. There has been rapid progress in recent years on the research of antimicrobial behavior of MOFs, along with the antimicrobial application of MOFs and their composites $[191,193]$. The antimicrobial mechanism of MOFs is mainly accredited to the inherent biocidal nature from their metal ions and may also be from the antimicrobial organic ligands [191, 193-195].

Ma et al. [195] combined MOFs and cellulose fibre (CF) via simple in situ generation and established multifunctional MOFs@CF air filters. The ZIF-8@CF filter exhibited high filtration efficiency of $98.36 \%$ against $0.3 \mu \mathrm{m}$ particles, high gas adsorption ability, and excellent antibacterial activity against
E. coli under a pressure drop of $134 \mathrm{~Pa}$. Very recently, inspired by the extremely tunable photocatalytic properties of MOFs, Li et al. exploited their photocatalytic biocidal activities and developed integrated air filters based on the MOFs (Figure 12) [196]. ZIF-8 nanocrystals were integrated to nonwoven fabrics via hot pressing. The established MOFilter achieved $96.8 \%$ removal of $\mathrm{PM}_{2.5}$ particles with a lowpressure drop (64 Pa) at a flow rate of $0.7 \mathrm{~m} \mathrm{~s}^{-1}$ and bactericidal efficiency higher than $99.99 \%$ over 30 min against aerosols containing E. coli. The dominant disinfection behavior of ZIF-8 here was ascribed to ROS production from photoelectrons trapped at $\mathrm{Zn}^{+}$centers within ZIF-8 via ligand to metal charge transfer (LMCT), rather than $\mathrm{Zn}^{2+}$ releasing. This work sheds light on the photocatalytic biocidal action of MOFs and provides valuable insights for their potential antimicrobial applications in air disinfection.

Though there have been numerous studies on antimicrobial materials and their antimicrobial application, the integration of antimicrobial substances with filters for air purification is still at a preliminary stage, especially for the newly emerging antimicrobial nanoparticles. In view of rising air pollution, and the severe 2019 coronavirus (COVID-19) pandemic, there would be increasing attention on air filters with antimicrobial properties. To achieve high-performance antimicrobial air filters, the choice of highly efficient and biologically safe antimicrobial ingredients, the structural design for good gas permeation, and a simple and economic preparation method are key for their ultimate commercialization to provide protection for public health. 
6.3. The Development of Masks with Antimicrobial Activity. While face masks can offer some form of protection against airborne and droplet-borne pathogens entering our airway through the mouth and nose, contact with the outer layer of the mask, such as with hands during mask adjustment, removal, or even disposal, can nonetheless result in selfinoculation of pathogenic microbes. A recent study showed that the SARS-CoV-2 virus can remain infectious on the outer layer of the surgical mask even after 6 days [197]. In addition, even accidentally touching a surface for as little as 5 seconds can result in the transfer of some quantity of the infectious microbes to the hands, for example, $32 \%$ of influenza A viruses [198]. Disinfection of masks, especially reusable ones, is therefore crucial. Cloth masks may be disinfected by washing with detergents and bleach whereas surgical and N95 masks may be disinfected via UV [199] or heat treatment $[116,200]$. Such treatments are nonetheless discrete, and masks are easily contaminated with pathogenic microbes again once in use. Thus, masks with antimicrobial activity that can automatically destroy or inactivate infectious microbes may reduce the risk of contamination.

With increased awareness of epidemics, research on methods to incorporate antimicrobial activity onto masks have intensified. Antimicrobial air filter materials discussed in the previous section (Section 6.2) can be extremely useful in this endeavour. Alternatively, masks may also be treated or coated with antimicrobial agents. Many classes of antimicrobial agents, including metal nanoparticles, organic compounds, and even common household chemicals, have demonstrated antimicrobial activity in masks. Notably, several of these masks are commercially available today; these examples will be highlighted in their respective sections. Some of these masks have also received FDA clearance as a single-use N95 respirator or surgical mask with antimicrobial/antiviral agent (ONT or OUK, respectively) [201, 202], which demonstrate significant antibacterial and antiviral activity in addition to satisfying the basic mask performance standards. Herein, some common antimicrobial agents that may be used to treat masks will be discussed.

6.3.1. Metal-Based Nanoparticles. Metal-based nanoparticles (NPs) are a growing field in the fight against microbes due to their low toxicity towards humans at concentrations effective for pathogen inactivation [203-207]. Due to their broad spectrum of biocidal activity and high potency, most do not induce resistance and are effective against multiresistant bacteria $[208,209]$. The two main mechanisms of biocidal activity are as follows: (i) heavy metal ions bind and precipitate thiol $(\mathrm{SH})$ groups in proteins, phosphate $\left(\mathrm{PO}_{4}^{-}\right)$groups in ATP and DNA, and other negatively charged groups in the cell wall/viral envelope, thus causing damage to key microbe functions; (ii) generation of reactive oxygen species (ROS) through changes in redox states or photocatalytic activity, which cause oxidative stress to the microbes.

(1) Silver Nanoparticles. Ag and its compounds have broadspectrum antimicrobial activities and have been widely applied as coatings to medical devices; the high affinity of $\mathrm{Ag}^{+}$to $\mathrm{SH}$ is the main mechanism of action [210-212]. There have been several reports of AgNPs conferring antimicrobial properties to masks. One method is to introduce AgNPs onto the materials used to make masks. US Pat. 6979491 disclosed the preparation of antimicrobial yarn by loading the fibrous material with glucose-capped AgNPs. The yarn showed antimicrobial activity against multiple bacteria genus, including Bacillus, Staphylococcus, Chlamydia, Escherichia, and Pseudomonas, and fungi, such as Candida albicans even after dying and 100 times of washing [213]. Anson Nanobiotechnology (Zhuhai) uses this fabric between the electret filter and the inner layer of the mask to produce nanosilver antibacterial surgical masks $[99,214]$. Other methods of loading AgNPs onto fabric for mask materials have been described [215-217]. A more facile method could be to coat AgNPs directly onto surgical masks; More et al. showed that masks soaked in a colloidal solution of starch-capped AgNPs possessed antimicrobial activity against both Gram positive $(S$. aureus) and Gram negative (E. coli) [218].

(2) Copper Nanoparticles. $\mathrm{Cu}$ and copper oxide both have potent biocidal properties and have been incorporated into textiles and other products with antimicrobial and antiviral properties [21, 219-223]. The main mechanism of action for CuNPs is the production of ROS during the oxidation of $\mathrm{Cu}(\mathrm{I})$. Borkow and coworkers from Cupron Inc. fabricated N95 masks with copper oxide $\left(\mathrm{Cu}_{2} \mathrm{O}\right.$ and $\left.\mathrm{CuO}\right)$ NPs in both the external and filtration layers [224]. These $\mathrm{Cu}_{\mathrm{x}} \mathrm{O}$-impregnated masks showed not only $99.85 \%$ viral filtration efficiencies of human influenza $\mathrm{A}$ and avian influenza viruses (similar to control) but also $99.99 \%$ reduction in virus titers on the mask surface after 30 minutes. The mask successfully passed European EN 14683:2005 and NIOSH N95 standards. Mask safety was also evaluated: the masks did not cause skin irritation nor poisoning through inhalation or saliva ingestion. A commercially available example is NBC Meshtec's Cufitec $^{\circledR}$ surgical mask, which contains $0.5 \mathrm{wt} \% \mathrm{CuI}$ in the outer layers [225]. The mask inactivated $99.99 \%$ of tested influenza A (H1N1, H2N2, H3N2, H5N1, and H5N9) and $\mathrm{B}$ viruses within 5 minutes of exposure and received FDA clearance as an antimicrobial mask (OUK) [226]. Copper (oxide) impregnated fibres have similarly been used in reusable masks. Cupron Inc. produces washable masks made of cotton and patented Cupron ${ }^{\circledR}$ polyester, comprising of $\mathrm{Cu}_{\mathrm{x}} \mathrm{O}$ NPs embedded in Rayon fibres [227-229]. Copperline's washable copper knit masks produced from LSK FineTex's patented copper fibres $[230,231]$ demonstrate $>92.3 \%$ virus and particulate filtration efficiency, which was increased to 99\% with HEPA filter (not reusable), and $>99 \%$ bacteria reduction after $60 \mathrm{~min}$ [232]. Copper Clothing Ltd. produces washable KN99 (FFP3) copper-infused masks; the outermost CuNP fabric was $>99 \%$ bacteriostatic to $S$. aureus and $P$. bacillus even after 50 washes (patents pending) [233, 234]. Several other copper fibre manufacturers, e.g., CuTEC ${ }^{\circledR}$, Kuhn Copper Solutions, and CoureTex ${ }^{\circledR}$, have either teamed up with garment manufacturers or produced copper masks themselves.

(3) Nanoparticles as Photocatalysts. Photocatalysts typically inactivate microbes by producing ROS via light-catalysed 
redox reactions [235-237]. Masks with a surface titanium oxide- $\left(\mathrm{TiO}_{2}^{-}\right)$apatite layer on the outer nonwoven fabric layer have shown good filtration and photocatalytic activity $[238,239]$. Zinc oxide $(\mathrm{ZnO})$ is used in commercially available machine-washable masks with $5 \mu \mathrm{m}$ particulate filtration developed by Sonovia; the inner polyester fabric of the mask is coated with $\mathrm{ZnO}$ NPs using patented ultrasonic cavitation technology, leading to a $98 \%$ reduction in surface bacteria E. coli and S. aureus after $1 \mathrm{~h}$ incubation [240, 241]. A recent study showed that even metal-organic frameworks (MOFs) could be used as mask filters. The Zn-imidazolate MOF filter showed $>99.99 \%$ photocatalytic bactericidal efficiency against E. coli aerosol after $30 \mathrm{~min}$ and 97\% PM removal. When incorporated into a 3-ply mask with nonwoven fabric outer layers, all layers showed almost no measurable level of viable bacteria after $30 \mathrm{~min}$ simulated sunlight illumination [196]. Despite the high antimicrobial activity of photocatalyst-impregnated masks, it must be noted that they are only effective when sufficient light energy is applied.

(4) Multiple Nanoparticle Species. The wide usage of some metal NPs, especially silver, has led to some bacterial resistance against these agents [242, 243]. Combinations of multiple NP species may be beneficial to the biocidal efficiency. Surgical masks with the outer hydrophobic layer coated with Ag and $\mathrm{TiO}_{2}$ NPs showed $100 \%$ reduction in viable E. coli and $S$. aureus after $48 \mathrm{~h}$ incubation while control masks showed a $25 \%$ and $50 \%$ increase in bacterial counts, respectively; the masks did not cause any skin inflammation or allergy [181]. Argaman Technologies, founded by Gabbay in 2016, produces BioBlockX ${ }^{\mathrm{TM}}$ face masks with Respilon ${ }^{\circledR}$ nanofibre filter membrane and 4 patented Argaman $\mathrm{Cu}$ infused layers. The $\mathrm{Cu}_{2} \mathrm{O}$ - and $\mathrm{Ag}_{4} \mathrm{O}_{4}$-impregnated fabrics inhibited $96 \%$ of the HIV-1 virus after $30 \mathrm{~min}$ and $86 \%$ of E. coli after $3 \mathrm{~h}$, which was significantly higher than fabrics with only $\mathrm{Cu}_{2} \mathrm{O}$ alone $[244,245]$. However, due to the nanofibre filter, the mask should not be laundered.

\subsubsection{Common Household Chemicals}

(1) Organic Acids. Acid-based media, such as citric acid, cause inactivation and aggregation of hemagglutinin (HA) glycoprotein spikes in virus membranes, thus rendering the virus unable to enter cells $[246,247]$. The application of citric acid as a coating on the outer layer of face masks was patented as early as 1989 [248] and is currently widely in use. A key example is the GlaxoSmithKline's Actiprotect ${ }^{\circledR}$ N95 respirator, which was the first to receive FDA clearance as an antimicrobial mask (ONT) in 2009 [249], with citric acid coating the outer polyester layer. In addition to passing standardized N95 tests, the Actiprotect ${ }^{\circledR}$ mask inactivated 99.99\% of tested influenza A (H1N1, H2N2, H3N2, H5N1, and H5N9) and B viruses within 1 minute of exposure [250].

(2) Sodium Chloride. Even coating the PP filtration layer with simple table salt $(\mathrm{NaCl})$ can confer virucidal activity [251]. $\mathrm{NaCl}$-coated filters showed increased filtration efficiency against $\mathrm{H} 1 \mathrm{~N} 1$ virus aerosols, as well as significantly lowering the virus titers after $5 \mathrm{~min}$ incubation; the proposed mecha- nism is damage to the virus membranes. In vivo studies with mice exposed to the $\mathrm{H} 1 \mathrm{~N} 1$ and $\mathrm{H} 5 \mathrm{~N} 1$ viruses through $\mathrm{NaCl}$ coated filters showed drastically reduced lung virus titer and increased survival rate compared to the control.

\subsubsection{Organic Compounds}

(1) Polyphenols. Tea polyphenols possess antiviral properties due to the ability of catechin, theaflavin, and their derivatives to damage virus membranes and bind to viral nucleic acids, inhibiting replication in influenza $A(\mathrm{H} 1 \mathrm{~N} 1$ and $\mathrm{H} 3 \mathrm{~N} 2)$ and $B$ viruses $[252,253]$. US patent 5888527 disclosed that dip coating the nonwoven fabric or electret filter of a mask into tea polyphenol extract can inactivate $>99 \%$ of tested viruses [254]. Catel-Ferreira et al. followed up by grafting catechin onto nonwoven cellulose (Kimberly-Clark ${ }^{\circledR}$ Kimwipes ${ }^{\circledR}$ Lite) using enzyme laccase [255]. While catechin at this concentration did not inhibit E. coli bacteria growth, it reduced the surface virus titer of T4D bacteriophage. When used as a filter layer in Kolmi M24001 mask, 99.99\% filtration of T4D was observed after $2 \mathrm{~h}$, a 7.5 times improvement over the original filter.

(2) Cationic Ammonium Compounds. The biocidal properties of 3-(trimethoxysilylpropyl)dimethyloctadecylammonium chloride and related organosilicon quaternary ammonium chloride- (Si-QAC-) treated surfaces (glass, stone, fibres, metals, and plastic) against bacteria, yeast, algae, and fungi were demonstrated as early as 1971 [256-258]; since then, it has been commercially marketed as Aegis ${ }^{\circledR}$ antimicrobial. Its use as a face mask coating has been disclosed [259, 260] and reusable masks are commercially available from Breathe Healthy ${ }^{\circledR}$ (mask biocidal activity not reported). Cationic ammonium polymers have also been studied: Tiliket et al. coated Kimwipes ${ }^{\circledR}$ with poly(ethyleneimine) (PEI) at $\mathrm{pH} 6$ to increase the protonated cationic form [261]. When used as a filter layer in a Kolmi M24001 mask, 99.999\% filtration of T4D bacteriophage was achieved in $1 \mathrm{~h}$. However, live virus was detected on the PEI-coated Kimwipes ${ }^{\circledR}$, indicating that the virus was captured but not killed.

(3) Polymers. The effectiveness of organic acids also led to the development of acidic polymers for mask materials. Dip coating the nonwoven PP filter layer of the mask, or spray coating the polyester outer layer of the mask with solutions of Carbopol ${ }^{\circledR}$ or Gantrez ${ }^{\mathrm{TM}}$ S-type polymers, resulted in up to $99.9 \%$ reduction in influenza A (H5N1) virus titer after 1 min incubation [262].

6.3.4. 2D Materials. 2D materials with a large latera size but atomic-scale thickness are advantageous for antimicrobial applications as their sharp edge has a nanoknife effect that can physically damage the bacterial cell, and some of them also possess outstanding photothermal and photocatalytic properties [263]. Graphene, the superstar of 2D materials, has been most explored as an antimicrobial in various areas [264]. Graphene and its derivatives have also been widely used with other antimicrobial agents, taking advantage of their large surface area, for a synergistic effect to enhance 
antimicrobial efficacy [265, 266]. Recently, the excellent photothermal properties of graphene in NIR regions have been utilized to increase the surface temperature and thus inactivate microorganisms [267, 268]. Other 2D materials, such as $\mathrm{MoS}_{2}$ [269-272] and graphitic carbon nitride (g$\mathrm{C}_{3} \mathrm{~N}_{4}$ ) [273-275], also show attractive antimicrobial performance, while their potential antimicrobial application in air filtration needs to be further explored.

6.3.5. Combination of Multiple Antimicrobial Classes. Several masks have also integrated more than one class of antimicrobial agents across multiple layers. US patent 7845351 disclosed that treating the outer nonwoven layer with antimicrobial agents comprising polyhexamethylene biguanide, citric acid, and $N$-alkyl polyglycoside, as well as other known antimicrobial agents, can deactivate $99.9 \%$ of treated bacteria (MRSA, vancomycin-resistant E. faecalis, M. catarrhalis, and K. pneumoniae), fungus C. albicans, and viruses (rhinovirus 1A, influenza A) within 30 minutes of contact [260]. US patent application US20110114095A1 disclosed the use of AgNP-impregnated activated carbon cloth (ACC) as the filtration layer in a face mask [276]; ACC itself showed antiviral activity (93\%) against MS-2 coliphage after $6 \mathrm{~h}$ incubation, which was enhanced by impregnation of AgNPs (98\%). Incorporation of the AgNPs/ACC into a mask resulted in $>99.88 \%$ virus filtration while having increased air permeability compared to the FFP3 mask. Some FDAcleared examples of antimicrobial mask include Filigent's BioFriend $^{\text {TM }}$ Biomask $^{\text {TM }}$ [277-279], cobranded with Medline Curad ${ }^{\circledR}$ Biomask $^{\mathrm{TM}}$ (ONT and OUK), as well as Innonix's RespoKare ${ }^{\mathrm{TM}}$ mask line (OUK child masks [280]), which both use $2 \mathrm{wt} \%$ citric acid on the outermost spunbound PP layer, as well as $\mathrm{Cu}$ (II) and $\mathrm{Zn}$ (II) (1.6 wt\% each) coordinated to sulfonated Rayon in a second layer before the melt-blown PP filter [281, 282]. Both antiviral masks inactivated $99.99 \%$ of tested influenza A (H1N1, H2N2, $\mathrm{H} 3 \mathrm{~N} 2, \mathrm{H} 5 \mathrm{~N} 1$, and $\mathrm{H} 5 \mathrm{~N} 9$ ) and B viruses within 5 minutes. Nexera Medical's SpectraShield ${ }^{\mathrm{TM}} 9500$ masks (ONT) [283] use patented $\mathrm{Ag}-\mathrm{Cu}$ zeolite $[284,285]$ (Agion ${ }^{\circledR}$, Sciessen LLC) in the outer PET fibre layer [286] (Fosshield ${ }^{\circledR}$, Foss Manufacturing); it kills $99.99 \%$ of tested bacteria (S. pyogenes, MRSA, and $H$. influenzae) and can also inactivate SARS, influenza, and filovirus. It has been tested for continuous use for up to 8 hours. Similar technology has also been described elsewhere [287].

In summary, multiple types of biocidal agents have been incorporated into masks, giving them the added ability to kill pathogenic microbes while not adversely affecting their basic performance. To date, many masks, some including more than one type of antimicrobial agent, are commercially available as summarised in Table 3. However, it must be noted that mask antimicrobial activities have only been studied under strict laboratory conditions; the actual performance of the masks during day-to-day usage may vary. For example, the time required to achieve high biocidal activity may be dependent on the amount of light, especially for photocatalytic biocidal agents, humidity, or airflow. The performance of reusable masks after repeated washing, especially after laundering with surfactants and at high temperatures, may also differ from the laboratory tests. Hence, while antimicrobial masks can offer additional protection against microbes, basic hygiene practices such as not touching the mask surface and washing of hands should still be observed.

6.4. Masks with Other Functional Properties. Increasingly, wearing of face masks is becoming the new norm in our lives. In an attempt to curb the spread of the virus, more than 50 countries have made face masks mandatory in public spaces during the COVID-19 pandemic, such as China, Singapore, Spain, and France [290]. In other places, medical experts highly encourage the use of face masks for the protection of the community and oneself against viral transmission. As international travel bans gradually lift, airlines require passengers to don masks at all times. Experts are anticipating a prolonged period of such measures as the world battles the disease. Even when we leave the shadows of the COVID-19 pandemic, we prepare and anticipate future health crises, especially those of a respiratory and infectious nature. We could perhaps no longer treat mask-wearing as a temporary solution but to adapt to having face masks as part of our staple of accessories. With that comes an array of peripheral issues and problems to address, not necessarily medical in nature, in the "new norm." The following paragraphs aim to highlight several of these. To note also is the matter of cost-as a temporary transient accessory or a surgical PPE, currently mask manufacturing tends to aim at driving the cost down, at producing cheap disposable units. With it becoming a staple, people may be convinced to invest more in sophisticated, multifunctional reusable variants, and cost-per-wear will help justify the addition of these attributes.

6.4.1. Super Hydrophobicity. In addition to their antimicrobial activities, functionalized graphene and graphene-based composites have been reported to confer superhydrophobicity onto material surfaces. Very recently, Zhong et al. [232] established the deposition of few-layer graphene onto commercial nonwoven masks via dual-mode laser-induced forward transfer. The graphene-deposited mask exhibited outstanding superhydrophobic and photothermal performance. The superhydrophobic surface of a graphene-coated mask can effectively repel the incoming aqueous droplets, while the as-coated mask surface can go up to $80^{\circ} \mathrm{C}$ under sunlight illumination to achieve self-sterilization. More impressively, the roll-to-roll laser production system can be integrated with current roll-to-roll surgical mask production lines, and the cost of raw materials is low, which makes the technology promising for commercial applications. Furthermore, the graphene-coated masks can be further recycled for solar-driven desalination.

6.4.2. Transparent Quality. There are three main categories of social needs that require a transparent quality to our everyday face mask: the hearing-impaired, the digital facerecognition technology, and the human-facing industry. There are an estimated 466 million in the world suffering from deafness or hearing loss [291], who heavily depend on lip-reading for communication. With the loss of partial face visibility, a substantial population of the hearing-impaired 
TABLe 3: Properties of commercially available antimicrobial masks.

\begin{tabular}{|c|c|c|c|c|c|}
\hline Mask brand & Classification $^{\mathrm{a}}$ & $\begin{array}{l}\text { Antimicrobial in outer } \\
\text { layer }\end{array}$ & $\begin{array}{l}\text { Antimicrobial in inner } \\
\text { layer }\end{array}$ & $\begin{array}{l}\text { Mask proven biocidal } \\
\text { against }\end{array}$ & Ref. \\
\hline $\begin{array}{l}\text { Nexera Medical } \\
\text { SpectraShield }\end{array}$ & N95 (ONT) & $\mathrm{Ag}$-Cu zeolite & $\mathrm{x}$ & Bacteria; virus & [283-286] \\
\hline $\begin{array}{l}\text { GlaxoSmithKline } \\
\text { Actiprotect }{ }^{\circledR}\end{array}$ & N95 (ONT) & Citric acid & $\mathrm{X}$ & Virus & {$[249,250]$} \\
\hline $\begin{array}{l}\text { Filigent BioFriend }{ }^{\mathrm{TM}} \\
\text { Biomask }^{\mathrm{TM}}\end{array}$ & $\begin{array}{c}\text { N95 (ONT) } \\
\text { Surgical (OUK) }\end{array}$ & Citric acid & $\begin{array}{l}\text { Cu NPs } \\
\text { Zn NPs }\end{array}$ & Virus & $\begin{array}{c}{[277-279,281,} \\
282]\end{array}$ \\
\hline Innonix RespoKare ${ }^{\mathrm{TM}}$ & Surgical (OUK) & Citric acid & $\begin{array}{l}\text { Cu NPs } \\
\text { Zn NPs }\end{array}$ & Virus & [280-282] \\
\hline NBC Meshtec Cufitec ${ }^{\circledast}$ & Surgical (OUK) & CuI NPs & $\mathrm{X}$ & Virus & {$[225,226]$} \\
\hline Anson Nano Silver & Surgical & $\mathrm{x}$ & AgNPs & Bacteria; fungi & {$[214,288]$} \\
\hline Copper Clothing & $\begin{array}{c}\text { Washable KN99 } \\
\text { (FFP3) }\end{array}$ & $\mathrm{Cu}_{\mathrm{x}} \mathrm{O}$ NPs & $\mathrm{X}$ & Bacteria; virus; fungi ${ }^{\mathrm{b}}$ & {$[233,234]$} \\
\hline Cupron Inc. & Washable & $\mathrm{Cu}_{\mathrm{x}} \mathrm{O}$ NPs & $\mathrm{X}$ & Bacteria; virus; fungi ${ }^{\mathrm{b}}$ & [227-229] \\
\hline Copperline & Washable $^{c}$ & $\mathrm{Cu}_{\mathrm{x}} \mathrm{O}$ NPs & $\mathrm{X}$ & Bacteria & {$[230,231,289]$} \\
\hline Argaman BioBlockX $\mathrm{X}^{\mathrm{TM}}$ & Reusable $^{\mathrm{d}}$ & $\begin{array}{l}\mathrm{Cu}_{2} \mathrm{O} \mathrm{NPs} \\
\mathrm{Ag}_{4} \mathrm{O}_{4} \mathrm{NPs}\end{array}$ & $\begin{array}{l}\mathrm{Cu}_{2} \mathrm{O} \text { NPs } \\
\mathrm{Ag}_{4} \mathrm{O}_{4} \mathrm{NPs}\end{array}$ & Bacteria; virus & {$[244,245]$} \\
\hline Sonovia Sonomask ${ }^{\mathrm{TM}}$ & Washable & $\mathrm{X}$ & $\mathrm{ZnO} N \mathrm{NPs}$ & Bacteria & {$[240,241]$} \\
\hline
\end{tabular}

${ }^{a}$ Medical and/or FDA classification. OUK = FDA clearance as a surgical mask with antimicrobial/antiviral agent; ONT = FDA clearance as an N95 mask with antimicrobial/antiviral agent. Washable/reusable masks do not require any certification. ${ }^{\mathrm{b}}$ Antimicrobial tests were performed only on fabric but not directly on the masks. ${ }^{\mathrm{c}}$ Single HEPA filter provided with the mask is not washable or reusable. ${ }^{\mathrm{d}}$ Reusable but not recommended to be laundered.

will be adversely affected in speech perception. The world is also increasingly dependent on digital facial feature recognition technology-be it in airport/border control, CCTV monitoring (for surveillance and security), or unlocking our mobile phones. Face recognition algorithms have not been optimised for the mask-wearing era, and the lack of which directly threatens societal security. Lastly, for the human-facing industry or population, reading of facial expressions can be crucial [292, 293], for instance, interpreters and translators, caretakers for people with illnesses, confusion and anxiety, customer-facing staff (including medical staff where medical miscommunication may occur), interacting with people who speak a different language, or the elderly and the young. Mehrabian and Ferris have popularised the importance of nonverbal communication: $55 \%$ of communication is visual [294]. Reading one's full face provides nontrivial cues to accessing another fellow human being.

As tech companies attempt to resolve the second category of digital recognition using enhanced algorithms (such as the Israeli Corsight and the Chinese Hanvon) for covered faces $[295,296]$, we envisage a transparent mask as a straightforward solution to the aforementioned needs. The challenge is threefold for a transparent mask material-it needs to be nonpermeable to liquids and fluids, it should provide twoway protection against transmission (of viral shedding), and it has to be breathable for human wear. There have been numerous transparent face mask patents in the market [297301], for instance, one from 2010 that in particular highlights a disposable transparent antimicrobial face mask [259]. For medical purposes, it consists of highly porous transparent film, with nonporous microvented laminae to provide controlled gas permeation (but liquid-proof). The transparent panel is said to be made from thermoplastic films such as polyethylene terephthalate (PET) and polyvinylidene fluoride (PVDF), with perforations and pleats incorporated. The antimicrobial used here is a Si-QAC biocide that provides mechanical contact kill, to prevent chemical leaching from conventional antimicrobials. It forms a durable coating on porous textiles and films, by initially bonding to the target surface (of the mask), and thereafter copolymerising between the target surface and itself, resulting in no "dislodgeable residue, odour, leaching, off-gassing, migration, or diffusion of the molecule."

Another interesting patent from 1984 points to a "transparent, odour-free face mask" [302]. The inventor J.H. Steinberg proposes using invisible or transparent materials and antibiotic/antiodour solutions with a transparent resin. The bactericides and deodorant solutions are either embedded in the resin or coated on the resin sheet. This thin flexible foraminous resin or sheet would then be shaped into a cup/mask form.

More recently, commercial product ClearMask claims to be the first fully transparent face mask, patent-pending at the time of writing [300], for ease of connecting and communicating with people, focusing on a human-centric experience. The patent application appears to describe a design where the transparent plastic piece can also act as an "impermeable barrier for air and/or other particulates," and breathability is afforded by airflow from the side of the mask (but preventing airflow from/to top and bottom of the mask) when worn. Other products have also appeared on the market, for instance, Shieldofglory's Transparent Hygenic Masks, covered under their 2013 patent [303], offering reusable (and refillable) solutions for preventing saliva or bacteria; however, their antiviral ability is not known. 
6.4.3. Comfort, Convenience, and Cleaning. With prolonged wearing of masks being necessary, such as for hours on flights and enclosed or confined spaces, offices, and workplaces, the inconvenience and discomfort, especially for children and the elderly, are amplified by current mask designs. It usually boils down to a trade-off between comfort/breathability and filtration power. For instance, Konda et al. surveyed common fabrics used in cloth masks and evaluated their aerosol filtration efficiency for particulate sizes of $10 \mathrm{~nm}$ to $10 \mu \mathrm{m}$ [95]. The studies found hybrid fabrics are better at filtering out particles, likely due to the combined mechanical and electrostatic filtration ability of the materials. Nonetheless, a maximum of $80 \%$ filtration efficiency for particles under $300 \mathrm{~nm}$ has been observed in that study, compared to $95 \%$ in N95 masks. Cloth or homemade masks also tend to fit less well, further reducing their filtration efficiencies. Other efficacy studies indicate a similar trend of commercial surgical masks being a preferred viral barrier to homemade masks [12, 17, 304].

Materials and designs promising of better breathability, durability, and comfort have been proposed and invented for mask use. Several suggest the use of microporous membranes, or films with interconnected pores, for ventilation and breathability. Increasing the space between the mask and nose/lip area and enhancing the softness/flexibility of the material, ear loops, and the weight of material can all contribute towards the overall comfort [305-311]. One interesting feature, for instance, is encapsulating discontinuous patterns of a phase change material aimed at cooling the microclimate of the inside of a mask, by $\sim 1-7^{\circ} \mathrm{C}$, or by $>30 \mathrm{~J}$ [312]. The invention gives an example of microencapsulated paraffin wax; the transition temperature of the phase change material can be chosen in the range of $25-29^{\circ} \mathrm{C}$. Heat from the person's exhalation is absorbed by the material, which melts in the process, henceforth reducing the rise in temperature in the small space. The material resolidifies as it releases the energy to the ambient air during inhalation. The process can then be cycled. Such material is coated in a discontinuous pattern so as not to reduce coverage of the liquid-resistant barrier material. A cooling function is especially critical in a tropical climate $[313,314]$. Singapore's Innosparks has produced an "AIR+ mask," incorporating a microfan onto an N95 face mask for the said purpose [315].

The "LMP S2" mask replaces the N95 construction for silicone in a streamlined design, claiming to ensure an airtight and more conformable fit, and is softer on one's skin. The reusable mask comes with removable filters. Another design by BDCI involves a simple 3D-printed skeleton to reinforce the mask shape firmly, preventing negative air pressure from collapsing the mask cup during inhalation, hence improving the ease of breathing [316].

The ability to self-clean is certainly desirable. Disposable masks are usually not environmentally friendly and require constant manufacturing and purchasing. Having to wash reusable masks daily (or more) would increasingly be out of sync with the population's lifestyle. Apart from the aforementioned antimicrobial properties, a few self-cleaning or self-sterilizing approaches are available for repeated prolonged or repeated use of masks. Stanford et al. demonstrate self-sterilizing laser-induced graphene (LIG) in air filters [266]. The LIG has a high surface area and traps microbes whose proliferation is inhibited on the graphene. Cycles of Joule heating then kills the microorganisms and pathogens, and the high thermal stability of LIG allows it to be reused. Arnusch et al. took this LIG and LIG-composite nanotechnology further, extensively applied to wastewater treatment and water filters, using an applied electrical potential to be bactericidal [317-320]. Since the onset of the COVID-19 pandemic, Arnusch et al. are trying to commercialize the technology onto a face mask ("Guardian G-Volt"), developed by the LIGC Applications company. The LIG self-disinfectant system can be plugged into a portable battery or a home-dock via a USB port, and an applied electrical potential "fully sterilizes" the mask for safe reuse [321]. Zhong et al. in a separate effort, developed a dual-mode laser-induced forward transfer method for depositing graphene layers onto low-melting temperature surgical masks. The graphene coating functions as an aqueous-resistant layer on the mask surface. The surface temperature also heats up easily and quickly to $>80^{\circ} \mathrm{C}$ under sunlight, naturally sterilizing itself, pushing towards reusable and recyclable graphene masks [232].

One other common approach is the use of ultraviolet (UV) light. One early demonstration by inventor Ricci showed a germicidal mask [322], where the air breathed in by the user has been exposed to inbuilt UV radiation, killing pathogens and viruses. Likewise, the user's exhaled air can also be disinfected before being released to the ambience. More recently, the leading wearable tech company Huami's new product in development, Aeri mask, has an inbuilt UV light that can disinfect the mask filters within $10 \mathrm{~min}$ when connected to a power supply via USB cables [323]. When realised, this would make efficient reusable face masks.

6.4.4. Good-to-Haves: Function, Fashion, and Future. We live in a constantly image-conscious world of consumerism, social media, and complicated human psyche. As we transit into the new postpandemic age, we cannot ignore the social and psychological aspects of donning additional apparel. Aesthetics, trends, and fashion are important factors in everyday living, as we already see reusable fabric masks sporting various patterns and designs. Multiple functions, way beyond the essential medical needs and convenience, would be desired. Masks now provide a ready platform to incorporate gadgets, electronics, good-to-haves, and a canvas for novel ideas. As our postpandemic lifestyle evolves, it is appropriate to consider the fashion, function, and future of masking up.

It is inspiring to take creative design approaches into consideration when evaluating the next-generation protective masks. Yanko Design has gathered many innovative technology ideas along this line. In one, VYZR Technologies create a product BioVYZR, a purified-air bubble around one's head worn like a vest-strap, with all-around antifogging visibility, safe space, filtered air as an N95 mask, and positive air pressure for easy breathing. The setup when charged can run for 8-12 hours and is said to be more spacious and comfortable [324]. Another designer Joe Doucet takes on a fashionable stand with a sleek face mask-shield-visor all in one [325]. 
In terms of functionality, smart mask designs move towards air quality sensors/monitors, biochemical or physical sensors, health trackers, and even bone conduction headphones, etc.

(1) Communication Tools. Several products on the market now have incorporated bone conduction headphones/earphones and even microphones onto the mask design [316, $326,327]$, currently aimed at the outdoor sports community. Such features also help to enhance communication between the everyday mask-wearing population, when our voices are muffled by the mask materials.

(2) Breath Sensors. There are several reports of nanosensors embedded in surgical masks for monitoring human breathing rate, most of which use materials which produce electrical outputs in response to humidity during human breathing [328-330]. The Laboratory for Embedded and Programmable Systems (UC Davis) has also embedded oxygen, carbon dioxide, and flow sensor into an elevation training mask (Training Mask 2.0 ${ }^{\circledR}$ ) [331]. Abnormal breath patterns can indicate poor health or even lung disease $[273,331]$.

(3) Usage and Air Quality Sensor. Xiaomi Inc. has patented a smart mask which can record wearing time, respiratory rate, and download pollution level data [332]. From these data, the mask can calculate its pollutant absorption quantity and signal to the user when the mask should be replaced; the mask is expected to be commercially available soon. Xiaomi's current product AirPOP mask, targeted at protection against airborne pollution, also has its app-accessible integrated sensor chip clipped onto the mask surface.

(4) Self-Powered Masks. Zhou et. al. developed a bifunctional mask with an electret filter layer capable of also acting as a nanogenerator, breath, and even mask life sensor [333]. Using negatively charged electrospun PEI as the nonwoven electret, $99.6 \%$ particle $(0.3 \mu \mathrm{m})$ removal efficiency was achieved. The PEI electret can be loosely sandwiched between 2 iron (Fe) net electrodes to form a nanogenerator and incorporated into a commercial mask; during exhalation, the airflow causes movement of the electret between the Fe electrodes, generating alternating electricity to power a small LCD screen, which can display the measured breathing rate. As the surface charge of the PEI electret decreases, removal efficiency and power generated will decrease; thus, when breathing can no longer power the LCD, the mask should be replaced. The mask has been tested for $40 \mathrm{~h}$ of continuous use.

(5) Microbe Detectors. Face mask sampling has been used by Williams and coworkers to detect M. tuberculosis in breath [334]. A gelatine membrane sampling matrix in the mask collects breath and sputum from the user over $8 \mathrm{~h}$, which is then sent for laboratory PCR testing. This method is noninvasive and enabled early detection of tuberculosis in people whose tuberculous burden is too low to be detected by conventional sputum tests. The team is now working on developing polyvinyl alcohol test strips for mask sampling for SARSCoV-2 virus to detect COVID-19 [335]. Another team of researchers led by Collins developed a paper-based colorimetric sensor for the detection of Zika virus RNA [121]. The time for Zika diagnosis was shortened to less than $3 \mathrm{~h}$ with this sensor. The scientists are now designing a face mask which can detect SARS-CoV-2 virus and produce a fluorescent output [336].

As inspiring as it appears, the market is still waiting for a truly multifunctional multipurpose product, or one that can be tailored to one's needs. The Chinese tech company Huami has recently announced their new design of a transparent N95-like mask (the "Aeri mask") that incorporates multiple functions such as unlocking phones with facial recognition while wearing it, self-cleaning, removable air filters (with each filter lasting 1.5 months), antifogging, and in-built fan for breathable comfort. The product is said to sport a modular design, therefore allowing for customizable features according to one's needs. Prototyping is in progress at the time of writing [323].

\section{Future Perspective}

Following the World Health Organization's (WHO) first recommendation of wearing face masks in the general public in early April 2020 as the coronavirus spread globally, the demand for face masks escalated. This generated a demand for raw materials and environmental impact. A recent study from UCL suggests that if every person in the UK uses one single-use mask each day for a year, 66,000 tonnes of contaminated plastic waste would be generated, without counting the waste from packaging [337]. Assuming the same disposal rate for every affected country, the medical waste generated at a global scale is going to be substantial, with a negative impact on our ecosystem and human health [338].

Apart from physical waste, greenhouse gas (GHG) emission across the mask life cycle is another concern. GHG is emitted at every stage of the face mask life cycle, from the production of polymer resin, nonwoven sheet conversion, face mask assembly, and transportation to the end of life (EoL) treatments by incineration or landfill. It was reported that $1 \mathrm{~kg}$ fossil fuel-based plastics could emit $4.1 \mathrm{~kg}$ of $\mathrm{CO}_{2}-$ equivalent $\left(\mathrm{CO}_{2} \mathrm{e}\right)$ over the total life cycle on average in 2015 , among which over $60 \%$, about $2.7 \mathrm{~kg} \mathrm{CO}_{2} \mathrm{e}$, was released from the polymer resin production stage [339]. Based on the UCL projection of 66,000 tonnes of mask waste generated each year in the UK at the current high mask disposable rate, 178,200 tonnes of GHG could be released into the environment per year solely from the resin production stage. The subsequent energy-intensive manufacturing process such as melt-blown, transportation, and incineration is expected to further increase the carbon footprint of mask to a large extend. To have a clear picture of the exact impact being imposed by face masks, comprehensive life cycle assessment (LCA), a well-adopted methodology for analysing the environmental impact of an industrial system from cradle to grave, should be used. There is no doubt that the sudden increase in mask manufacturing and usage will cast more pressure to the already alarming global environmental issues originated from plastic products. 
On the one hand, more masks are needed for reducing the risk of the virus spreading (part of the preparations for economic reopening). On the other hand, reducing mask production, usage, and disposal is preferred for environmental reasons. Facing such a dilemma, tackling both challenges may need synergistic efforts from policymakers, industry players, researchers, and the general public. These efforts include strategies to reuse disposable masks, a search for alternative mask material with low GHG emission, etc. Amid the crisis, we are seeing promising initiatives from the industrial and research communities.

The reuse of single-use face masks provides a straightforward way to reduce the disposal rate. In this regard, appropriate disinfection processes for safe and frequent reuse of masks without composing the mask filtration efficiency need to be determined. This has been discussed in detail in the previous section (Section 5). Besides recycling commercial masks, DIY reusable masks also help to alleviate the supply shortage, with possible environmental benefits. As discussed in Section 4, with the right choice of materials, appropriate design, and assembly, a DIY reusable mask may function as well as commercially manufactured surgical ones. Using easily available household materials such as reusable nonwoven bag, dried hypoallergenic wet wipe, and a thin cotton cloth as the outer hydrophobic, middle filtering, and inner adsorbent layers, respectively, researchers from A*STAR, Singapore, successfully designed a DIY mask with essential properties comparable to a surgical mask [340]. As the performance of DIY masks depends on the materials used and how it is they are assembled, the public should not have the misconception that DIY masks can serve as a surgical mask alternative.

A changeable filter layer that can be fitted inside the masks prevails as a viable option in improving the performance of a self-improvised mask. The replaceable layer of fibrous material filters out viruses and other pathogens and allows the mask to be washed and reused. The team led by Jung at KAIST developed a versatile nanofibre filter that can be fitted inside a mask using an "Insulated Block Electrospinning (IBE)" [341]. The changeable filter endows the various grass-root production of masks over the past few months with up-to-standard filtration performance.

In addition to the essential filtering function, incorporating multifunctionality into mask design or mask materials opens up opportunities for masks with advanced features. "Self-sanitising" and "self-cleaning" are among the new terminologies being used in the future "greener" mask. Some of these innovative efforts have been described in Section 6.4.

Spurred by the outbreak of COVID-19, many companies are transitioning to mask production to meet the vast demand, such as global gaming hardware manufacturing company Razer Inc. Yet, this ambitious effort has run into a bottleneck, the deficit of melt-blown fabric. Putting supply availability aside, the life cycle GHG emission level of the raw materials poses another set of challenges. A remedy to these problems could be found in the development of "green" substitute products based on the polymer with lower GHG emission level or bio-based materials produced from plants.

For instance, Krucińska et al. earlier developed biodegradable particle-filtering respiratory half-masks from nonwoven poly(lactic acid) (PLA) which met the requirements of the low (FFP1) and medium (FFP2) protection classes against harmful aerosols [342]. Zhang et al. also reported the development of biodegradable electrospun poly(l-lactic acid) (PLLA) polymer nanofibres that can significantly improve the removal of $\mathrm{PM}_{2.5}$ particulates via the generation of electrostatic charges. The filter membrane exhibited a high efficiency of $99.3 \%$ and compared to a $3 \mathrm{M}$ respirator, still exhibits a $15 \%$ improvement in quality factor after 6 hours of filtration time [343]. Guzdemir and Ogale recently reported producing fibres with reduced content of PP for disposable fabrics by incorporating bio-based renewable material, soy flour, where thin fibres with a diameter under $60 \mu \mathrm{m}$ can be successfully meltspun [344]. It was found that the presence of soy particulates on fibre surface enhanced its water absorption and colourability properties while retaining the feel of natural fibres. More recently, researchers from the BioProducts Institute at the University of British Columbia have developed a fully compostable and biodegradable medical N95 mask, named CanMask, using wood fibres from sources such as pine, spruce, cedar, and other softwoods [345].

Although a large range of thermoplastic resins can be processed by spun-bond and melt-blown technology, an essential part of the mask structure, PP remains the major resin being used for its ease in processing, especially in terms of viscosity control. Hence, proper control of the rheological behavior using modifiers will assist in the production of nonPP melt-blown fabric that meets the requirement for medical applications. Natural materials such as cellulose, cotton, and commercial resins such as Bioplast could become potential candidates after issues regarding their poor thermal stability have been addressed.

Recent efforts have demonstrated the potential of reusable mask development enabled by material innovation and technology advancement in addressing the mask shortage while reducing the GHG emissions and negative environmental impact. However, continuous efforts are needed to ensure feasible developments can be transit to existing manufacturing facilities. Also, there are more scientific opportunities to develop novel and environmentally friendly mask materials with functions of interests such as self-sanitising and degradable materials and to develop a low energy consumption technique or process for a nonwoven fibre that could replace a carbon-intensive melt-blown process in the near future. This does not undermine the role of a single-use mask as an immediate measure to protect those at high risk of infection healthcare professionals. Till date, COVID-19 is still on the rise worldwide, the use of mask may become a norm and the demand will remain high. Hence, continuous efforts are required for a closely integrated experimental and theoretical investigation aiming to progress upon the current state of understanding and perpetuate the development of innovative solutions for the mask crisis amid the pandemic.

\section{Conclusion}

The COVID-19 pandemic has forced the global population to adopt new ways of living, including the wearing of masks as a new norm. It has even accelerated R\&D efforts in mask 
materials and design to offer better protection for users against airborne pollutants and pathogens. This review therefore provides a holistic summary of the A to $\mathrm{Z}$ of face masks, to give readers a broad-view understanding of masks from the perspective of public health to the domains of material development. The importance of mask-wearing in preventing the spread of airborne and droplet-borne infections was discussed early in this review. Thereafter, the protection mechanism, production, and performance testing of commercial masks were described. We then explored the effectiveness of DIY homemade masks as an alternative to commercial masks. To overcome the issue of mask shortage, methods to decontaminate used masks were introduced and elaborated. The review then discussed research advances in the development of materials with improved filtering capacity and antimicrobial activity. This was followed by R\&D efforts in the engineering of multifunctional masks with properties such as antimicrobial activity, hydrophobicity, transparent see-through surfaces, sensing-cleaning, selfpowered, and even sensing and detection capabilities. Finally, the environmental implications of widespread mask-wearing and increased mask production were deliberated upon as efforts towards finding more sustainable solutions to support long-term mask-wearing, even after the end of the pandemic, were explored. As mentioned in the very beginning, the fight against any infectious diseases requires efforts and solutions in prevention, detection, diagnosis, and treatment. The wearing of masks therefore serves as a key strategy towards airborne disease prevention that cannot be easily substituted.

\section{Conflicts of Interest}

The authors declare no conflict of interests.

\section{Authors' Contributions}

Ming Hui Chua, Weiren Cheng, Shermin Simin Goh, Junhua Kong, Bing Li, Jason Y. C. Lim, Lu Mao, Suxi Wang, Kun Xue, Le Yang, Enyi Ye, and Kangyi Zhang contributed equally to this work.

\section{References}

[1] S. Y. Wong and B. H. Tan, "Megatrends in infectious diseases: the next 10 to 15 years," Annals of the Academy of Medicine, Singapore, vol. 48, no. 6, pp. 188-194, 2019.

[2] M. Xie and Q. Chen, "Insight into 2019 novel coronavirus an updated interim review and lessons from SARS-CoV and MERS-CoV," International Journal of Infectious Diseases, vol. 94, pp. 119-124, 2020.

[3] W.-j. Guan, Z.-y. Ni, Y. Hu et al., "Clinical characteristics of coronavirus disease 2019 in China," New England Journal of Medicine, vol. 382, no. 18, pp. 1708-1720, 2020.

[4] S. G. Benzell, A. Collis, and C. Nicolaides, "Rationing social contact during the COVID-19 pandemic: transmission risk and social benefits of US locations," Proceedings of the National Academy of Sciences, vol. 117, no. 26, pp. 1464214644, 2020.

[5] D. K. Chu, E. A. Akl, S. Duda et al., "Physical distancing, face masks, and eye protection to prevent person-to- person transmission of SARS-CoV-2 and COVID-19: a systematic review and meta- analysis," The Lancet, vol. 395, no. 10242, pp. 1973-1987, 2020.

[6] J. W. Tang, T. J. Liebner, B. A. Craven, and G. S. Settles, “A schlieren optical study of the human cough with and without wearing masks for aerosol infection control," Journal of the Royal Society Interface, vol. 6, Supplement 6, pp. S727-S736, 2009.

[7] C. C. Leung, T. H. Lam, and K. K. Cheng, "Mass masking in the COVID-19 epidemic: people need guidance," The Lancet, vol. 395 , no. 10228 , p. $945,2020$.

[8] D. He, S. Zhao, Q. Lin et al., "The relative transmissibility of asymptomatic COVID-19 infections among close contacts," International Journal of Infectious Diseases, vol. 94, pp. 145-147, 2020.

[9] M. Gandhi, D. S. Yokoe, and D. V. Havlir, "Asymptomatic transmission, the Achilles' heel of current strategies to control Covid-19," New England Journal of Medicine, vol. 382, no. 22, pp. 2158-2160, 2020.

[10] Z. Gao, Y. Xu, C. Sun et al., "A systematic review of asymptomatic infections with COVID-19," Journal of Microbiology, Immunology and Infection, 2020.

[11] Y. Long, T. Hu, L. Liu et al., "Effectiveness of N95 respirators versus surgical masks against influenza: a systematic review and meta-analysis," Journal of Evidence-Based Medicine, vol. 13, no. 2, pp. 93-101, 2020.

[12] A. Davies, K.-A. Thompson, K. Giri, G. Kafatos, J. Walker, and A. Bennett, "Testing the efficacy of homemade masks: would they protect in an influenza pandemic?," Disaster Medicine and Public Health Preparedness, vol. 7, no. 4, pp. 413-418, 2013.

[13] A. V. Mueller, M. J. Eden, J. J. Oakes, C. Bellini, and L. A. Fernandez, "Quantitative method for comparative assessment of particle filtration efficiency of fabric masks as alternatives to standard surgical masks for PPE," medRxiv, 2020, 2020.04.17.20069567.

[14] M. van der Sande, P. Teunis, and R. Sabel, "Professional and home-made face masks reduce exposure to respiratory infections among the general population," PLoS One, vol. 3, no. 7, article e2618, 2008.

[15] S. D. Rubbo and L. R. Abbott, "Filtration efficiency of surgical masks: a new method of evaluation," Australian and New Zealand Journal of Surgery, vol. 38, no. 1, pp. 8083, 1968.

[16] N. Zhu, D. Zhang, W. Wang et al., "A novel coronavirus from patients with pneumonia in China, 2019," New England Journal of Medicine, vol. 382, no. 8, pp. 727-733, 2020.

[17] N. H. L. Leung, D. K. W. Chu, E. Y. C. Shiu et al., "Respiratory virus shedding in exhaled breath and efficacy of face masks," Nature Medicine, vol. 26, no. 5, pp. 676-680, 2020.

[18] S. E. Eikenberry, M. Mancuso, E. Iboi et al., “To mask or not to mask: modeling the potential for face mask use by the general public to curtail the COVID-19 pandemic," Infectious Disease Modelling, vol. 5, pp. 293-308, 2020.

[19] G. R. J. Swennen, L. Pottel, and P. E. Haers, "Custom-made $3 \mathrm{D}$-printed face masks in case of pandemic crisis situations with a lack of commercially available FFP2/3 masks," International Journal of Oral and Maxillofacial Surgery, vol. 49, no. 5, pp. 673-677, 2020.

[20] J. S. Kutter, M. I. Spronken, P. L. Fraaij, R. A. M. Fouchier, and S. Herfst, "Transmission routes of respiratory viruses 
among humans," Current Opinion in Virology, vol. 28, pp. 142-151, 2018.

[21] N. van Doremalen, T. Bushmaker, D. H. Morris et al., "Aerosol and surface stability of SARS-CoV-2 as compared with SARS-CoV-1," New England Journal of Medicine, vol. 382, no. 16, pp. 1564-1567, 2020.

[22] C. B. Hall and R. G. Douglas, "Modes of transmission of respiratory syncytial virus," The Journal of Pediatrics, vol. 99, no. 1, pp. 100-103, 1981.

[23] J. M. Leclair, J. Freeman, B. F. Sullivan, C. M. Crowley, and D. A. Goldmann, "Prevention of nosocomial respiratory syncytial virus infections through compliance with glove and gown isolation precautions," New England Journal of Medicine, vol. 317, no. 6, pp. 329-334, 1987.

[24] R. Pung, C. J. Chiew, B. E. Young et al., "Investigation of three clusters of COVID-19 in Singapore: implications for surveillance and response measures," The Lancet, vol. 395, no. 10229, pp. 1039-1046, 2020.

[25] L. Bourouiba, "Turbulent gas clouds and respiratory pathogen emissions: potential implications for reducing transmission of COVID-19," JAMA, vol. 323, no. 18, pp. 1837-1838, 2020.

[26] T. Dbouk and D. Drikakis, "On coughing and airborne droplet transmission to humans," Physics of Fluids, vol. 32, no. 5, article 053310, 2020.

[27] E. N. Perencevich, D. J. Diekema, and M. B. Edmond, "Moving personal protective equipment into the community," JAMA, vol. 323, no. 22, pp. 2252-2253, 2020.

[28] P. Anfinrud, V. Stadnytskyi, C. E. Bax, and A. Bax, "Visualizing speech-generated oral fluid droplets with laser light scattering," New England Journal of Medicine, vol. 382, no. 21, pp. 2061-2063, 2020.

[29] V. Stadnytskyi, C. E. Bax, A. Bax, and P. Anfinrud, "The airborne lifetime of small speech droplets and their potential importance in SARS-CoV-2 transmission," Proceedings of the National Academy of Sciences, vol. 117, no. 22, pp. 11875-11877, 2020.

[30] L. Morawska, G. R. Johnson, Z. D. Ristovski et al., "Size distribution and sites of origin of droplets expelled from the human respiratory tract during expiratory activities," Journal of Aerosol Science, vol. 40, no. 3, pp. 256-269, 2009.

[31] S. Asadi, A. S. Wexler, C. D. Cappa, S. Barreda, N. M. Bouvier, and W. D. Ristenpart, "Aerosol emission and superemission during human speech increase with voice loudness," Scientific Reports, vol. 9, no. 1, p. 2348, 2019.

[32] R. B. Patel, S. D. Skaria, M. M. Mansour, and G. C. Smaldone, "Respiratory source control using a surgical mask: an in vitro study," Journal of Occupational and Environmental Hygiene, vol. 13, no. 7, pp. 569-576, 2016.

[33] D. F. Johnson, J. D. Druce, C. Birch, and M. L. Grayson, “A quantitative assessment of the efficacy of surgical and N95 masks to filter influenza virus in patients with acute influenza infection," Clinical Infectious Diseases, vol. 49, no. 2, pp. 275277, 2009.

[34] D. K. Milton, M. P. Fabian, B. J. Cowling, M. L. Grantham, and J. J. McDevitt, "Influenza virus aerosols in human exhaled breath: particle size, culturability, and effect of surgical masks," PLoS Pathogens, vol. 9, no. 3, article e1003205, 2013.

[35] W. H. Seto, D. Tsang, R. W. H. Yung et al., "Effectiveness of precautions against droplets and contact in prevention of nosocomial transmission of severe acute respiratory syndrome (SARS)," The Lancet, vol. 361, no. 9368, pp. 15191520, 2003.

[36] L. J. Radonovich Jr., M. S. Simberkoff, M. T. Bessesen et al., "N95 respirators vs medical masks for preventing influenza among health care personnel: a randomized clinical trial," JAMA, vol. 322, no. 9, pp. 824-833, 2019.

[37] V. Offeddu, C. F. Yung, M. S. F. Low, and C. C. Tam, "Effectiveness of masks and respirators against respiratory infections in healthcare workers: a systematic review and metaanalysis," Clinical Infectious Diseases, vol. 65, no. 11, pp. 1934-1942, 2017.

[38] W. E. Wei, Z. Li, C. J. Chiew, S. E. Yong, M. P. Toh, and V. J. Lee, "Presymptomatic transmission of SARS-CoV-2 - Singapore, January 23-March 16, 2020," Morbidity and Mortality Weekly Report, vol. 69, no. 14, pp. 411-415, 2020.

[39] M. Klompas, C. A. Morris, J. Sinclair, M. Pearson, and E. S. Shenoy, "Universal masking in hospitals in the Covid-19 era," New England Journal of Medicine, vol. 382, no. 21, article e63, 2020.

[40] K. H. Chan and K.-Y. Yuen, "COVID-19 epidemic: disentangling the re-emerging controversy about medical facemasks from an epidemiological perspective," International Journal of Epidemiology, 2020.

[41] S. Feng, C. Shen, N. Xia, W. Song, M. Fan, and B. J. Cowling, "Rational use of face masks in the COVID-19 pandemic," The Lancet Respiratory Medicine, vol. 8, no. 5, pp. 434-436, 2020.

[42] T. F. Tay, Coronavirus: mandatory for all in Singapore to wear mask when out, except for kids under 2 and those doing strenuous exercise, The Straits Times, 2020.

[43] Y. Bai, L. Yao, T. Wei et al., "Presumed asymptomatic carrier transmission of COVID-19," JAMA, vol. 323, no. 14 , pp. 1406-1407, 2020.

[44] X. Pan, D. Chen, Y. Xia et al., "Asymptomatic cases in a family cluster with SARS-CoV-2 infection," The Lancet Infectious Diseases, vol. 20, no. 4, pp. 410-411, 2020.

[45] A. Kimball, K. M. Hatfield, M. Arons et al., "Asymptomatic and presymptomatic SARS-CoV-2 infections in residents of a long-term care skilled nursing facility - King County, Washington, March 2020," Morbidity and Mortality Weekly Report, vol. 69, no. 13, pp. 377-381, 2020.

[46] CDC, Recommendation regarding the use of cloth face coverings, especially in areas of significant community-based transmission, Center for Disease Control and Prevention, 2020.

[47] C. R. MacIntyre, H. Seale, T. C. Dung et al., "A cluster randomised trial of cloth masks compared with medical masks in healthcare workers," BMJ Open, vol. 5, no. 4, article e006577, 2015.

[48] B. J. Cowling, K.-H. Chan, V. J. Fang et al., "Facemasks and hand hygiene to prevent influenza transmission in households," Annals of Internal Medicine, vol. 151, no. 7, pp. 437-446, 2009.

[49] A. E. Aiello, G. F. Murray, V. Perez et al., "Mask use, hand hygiene, and seasonal influenza-like illness among young adults: a randomized intervention trial," The Journal of Infectious Diseases, vol. 201, no. 4, pp. 491-498, 2010.

[50] C. R. MacIntyre, S. Cauchemez, D. E. Dwyer et al., "Face mask use and control of respiratory virus transmission in households," Emerging Infectious Diseases, vol. 15, no. 2, pp. 233-241, 2009. 
[51] R. Li, S. Pei, B. Chen et al., "Substantial undocumented infection facilitates the rapid dissemination of novel coronavirus (SARS-CoV-2)," Science, vol. 368, no. 6490, pp. 489-493, 2020.

[52] J. Spector, New York releases antibody testing data: 14\% of population may be infected with coronavirus, USA Today, 2020.

[53] J. Naftulin, WHO says there is no need for healthy people to wear face masks, days after the CDC told all Americans to cover their faces, Business Insider US, 2020.

[54] A. D. Sung, J. A. M. Sung, S. Thomas et al., "Universal mask usage for reduction of respiratory viral infections after stem cell transplant: a prospective trial," Clinical Infectious Diseases, vol. 63, no. 8, pp. 999-1006, 2016.

[55] S. M. Tracht, S. Y. Del Valle, and J. M. Hyman, "Mathematical modeling of the effectiveness of facemasks in reducing the spread of novel influenza A (H1N1)," PLoS One, vol. 5, no. 2, article e9018, 2010.

[56] D. Kai, G.-P. Goldstein, A. Morgunov, V. Nangalia, and A. Rotkirch, "Universal masking is urgent in the COVID-19 pandemic: SEIR and agent based models, empirical validation, policy recommendations," arXiv Physics and Society (pre-print), 2020.

[57] H. Lim, "A review of spun bond process," Journal of Textile and Apparel, Technology and Management, vol. 6, pp. 1-13, 2010.

[58] V. Midha and A. Dakuri, "Spun bonding technology and fabric properties: a review," Journal of Textile Engineering \& Fashion Technology, vol. 1, no. 4, 2017.

[59] I. M. Hutten, "Chapter 1 - introduction to nonwoven filter media," in Handbook of Nonwoven Filter Media (Second Edition), I. M. Hutten, Ed., pp. 1-52, Butterworth-Heinemann, Oxford, 2016.

[60] K. Dutton, "Overview and analysis of the meltblown process and parameters," Journal of Textile and Apparel, Technology and Management, vol. 6, no. 1, 2008.

[61] J. Hagewood, "3 - technologies for the manufacture of synthetic polymer fibers," in Advances in Filament Yarn Spinning of Textiles and Polymers, D. Zhang, Ed., pp. 48-71, Woodhead Publishing, 2014.

[62] B. Henneberry, "How surgical masks are made," https://www .thomasnet.com/articles/other/how-surgical-masks-aremade/.

[63] ASTM, F2100-19e1, Standard Specification for Performance of Materials Used in Medical Face Masks, ASTM International, West Conshohocken, PA, USA, 2019.

[64] Guidance Document on "Surgical Masks - Premarket Notification [510(k)] Submissions", U.S. Food and Drug Administration, 2004.

[65] ASTM, F2299 / F2299M-03(2017), Standard Test Method for Determining the Initial Efficiency of Materials Used in Medical Face Masks to Penetration by Particulates Using Latex Spheres, ASTM International, West Conshohocken, PA, USA, 2017.

[66] ASTM, F2101-19, Standard Test Method for Evaluating the Bacterial Filtration Efficiency (BFE) of Medical Face Mask Materials, Using a Biological Aerosol of Staphylococcus aureus, ASTM International, West Conshohocken, PA, USA, 2019.

[67] R. Valaperta, M. R. Tejada, M. Frigerio et al., "Staphylococcus aureus nosocomial infections: the role of a rapid and low-cost characterization for the establishment of a surveillance system," The New Microbiologica, vol. 33, no. 3, pp. 223-232, 2010.

[68] E. Klein, D. Smith, and R. Laxminarayan, "Hospitalizations and deaths caused by methicillin-resistant Staphylococcus aureus, United States, 1999-2005," Emerging Infectious Diseases, vol. 13, pp. 1840-1846, 2007.

[69] "Bacterial filtration efficiency (BFE) / virus filtration efficiency (VFE) test," May 2020, https://www.kaken.or.jp/test/ search/detail/34\#ID1.

[70] V. W. Greene and D. Vesley, "Method for evaluating effectiveness of surgical masks," Journal of Bacteriology, vol. 83, no. 3, pp. 663-667, 1962.

[71] "Mask protection standards \& medical face mask information for use," May 2020, https://www.primed.ca/clinicalresources/astm-mask-protection-standards/.

[72] N. Labs, "Bacterial \& viral filtration efficiency (BFE/VFE)," May 2020, https://www.nelsonlabs.com/testing/bacterialviral-filtration-efficiency-bfe-vfe/.

[73] "GlaxoSmithKline 510(k) summary - Actiprotect UF N95 respirator,” May 2020, http://www.accessdata.fda.gov/cdrh_ docs/pdf8/K081923.pdf.

[74] ASTM, F1862 / F1862M-17, Standard Test Method for Resistance of Medical Face Masks to Penetration by Synthetic Blood (Horizontal Projection of Fixed Volume at a Known Velocity), ASTM International, West Conshohocken, PA, USA, 2017.

[75] "MIL-M-36954C, military specification - mask, surgical, disposable," May 2020, http://www.frazierinstrument.com/ reference/standards/organizations/mil/abstract-mil-m36954c.html.

[76] Laboratory Test Manual for 16 CFR Part 1610: Standard for the Flammability of Clothing Textiles United States Consumer Product Safety Commission, Consumer Product Safety Commission (CPSC), 2008.

[77] N. Labs, "Flammability test," May 2020, https://www .nelsonlabs.com/testing/flammability-test/.

[78] "ISO 10993-5:2009 biological evaluation of medical devices - part 5: tests for in vitro cytotoxicity," 2009.

[79] "ISO 10993-10:2010 biological evaluation of medical devices - part 10: tests for irritation and skin sensitization," 2010.

[80] C. Carias, G. Rainisch, M. Shankar et al., "Potential demand for respirators and surgical masks during a hypothetical influenza pandemic in the United States," Clinical Infectious Diseases, vol. 60, Supplement 1, pp. S42-S51, 2015.

[81] B. Javid, M. P. Weekes, and N. J. Matheson, "Covid-19: should the public wear face masks?," BMJ (British Medical Journal), vol. 369, p. 2, 2020.

[82] D. Ramakrishnan, "COVID-19 and face masks - to use or not to use!," Indian Journal of Community Health, vol. 32, no. 2, pp. 240-243, 2020.

[83] S. Rengasamy, B. Eimer, and R. E. Shaffer, "Simple respiratory protection-evaluation of the filtration performance of cloth masks and common fabric materials against 20-1000 $\mathrm{nm}$ size particles," The Annals of Occupational Hygiene, vol. 54, no. 7, pp. 789-798, 2010.

[84] A. Patel, L. Lee, S. K. Pillai, A. L. Valderrama, L. J. Delaney, and L. Radonovich, "Approach to prioritizing respiratory protection when demand exceeds supplies during an influenza pandemic: a call to action," Health Security, vol. 17, no. 2, pp. 152-155, 2019. 
[85] J. A. CAPPS, "Measures for the prevention and control of respiratory infections in military camps," Journal of the American Medical Association, vol. 71, no. 6, pp. 448-451, 1918.

[86] D. Tham, N. Hien, P. Nga et al., "Use of cloth masks among healthcare workers in hospitals in Hanoi," Taåp chñ Y hoåc dûå phoâng, vol. 22, pp. 104-110, 2012.

[87] P. Yang, H. Seale, C. Raina MacIntyre et al., "Mask-wearing and respiratory infection in healthcare workers in Beijing, China," The Brazilian Journal of Infectious Diseases, vol. 15, no. 2, pp. 102-108, 2011.

[88] A. A. Chughtai, H. Seale, and C. R. MacIntyre, "Use of cloth masks in the practice of infection control - evidence and policy gaps," International Journal of Infection Control, vol. 9, no. 3, 2013.

[89] A. A. Chughtai, H. Seale, T. C. Dung, A. Hayen, B. Rahman, and C. Raina MacIntyre, "Compliance with the use of medical and cloth masks among healthcare workers in Vietnam," The Annals of Occupational Hygiene, vol. 60, no. 5, pp. 619630, 2016.

[90] K. M. Shakya, A. Noyes, R. Kallin, and R. E. Peltier, "Evaluating the efficacy of cloth facemasks in reducing particulate matter exposure," Journal of Exposure Science \& Environmental Epidemiology, vol. 27, no. 3, pp. 352-357, 2017.

[91] B. B. Neupane, S. Mainali, A. Sharma, and B. Giri, "Optical microscopic study of surface morphology and filtering efficiency of face masks," PeerJ, vol. 7, pp. e7142-e7142, 2019.

[92] M. Sugrue, D. O'Keeffe, R. Sugrue, L. MacLean, and M. Varzgalis, "A cloth mask for under-resourced healthcare settings in the COVID19 pandemic," Irish Journal of Medical Science, 2020.

[93] T. Ma and N. Shutler, How to Sew a Fabric Face Mask. The New York Times, 2020, https://www.nytimes.com/article/ how-to-make-face-mask-coronavirus.html.

[94] Centers for Disease Control and Prevention, Use of Cloth Face Coverings to Help Slow the Spread of COVID-19. Coronavirus Disease 2019 (COVID-19) 2020, CS316353B, 1 3https://www.cdc.gov/coronavirus/2019-ncov/preventgetting-sick/diy-cloth-face-coverings.html.

[95] A. Konda, A. Prakash, G. A. Moss, M. Schmoldt, G. D. Grant, and S. Guha, "Aerosol filtration efficiency of common fabrics used in respiratory cloth masks," ACS Nano, vol. 14, no. 5, pp. 6339-6347, 2020.

[96] J. C. Rubio-Romero, M. C. Pardo-Ferreira, J. A. TorrecillaGarcía, and S. Calero-Castro, "Disposable masks: disinfection and sterilization for reuse, and non-certified manufacturing, in the face of shortages during the COVID-19 pandemic," Safety Science, vol. 129, article 104830, 2020.

[97] M. Ippolito, F. Vitale, G. Accurso et al., "Medical masks and respirators for the protection of healthcare workers from SARS-CoV-2 and other viruses," Pulmonology, vol. 26, no. 4, pp. 204-212, 2020.

[98] A. Polkinghorne and J. Branley, "Evidence for decontamination of single-use filtering facepiece respirators," The Journal of Hospital Infection, vol. 105, no. 4, pp. 663669, 2020.

[99] Y. Zhao, Z. Zhao, Y. Wang, Y. Zhou, Y. Ma, and W. Zuo, Single-cell RNA expression profiling of ACE2, the putative receptor of Wuhan 2019-nCov, bioRxiv, 2020.

[100] D. J. Viscusi, M. S. Bergman, B. C. Eimer, and R. E. Shaffer, "Evaluation of five decontamination methods for filtering facepiece respirators," The Annals of Occupational Hygiene, vol. 53, no. 8, pp. 815-827, 2009.

[101] D. J. Viscusi, W. P. King, and R. E. Shaffer, "Effect of decontamination on the filtration efficiency of two filtering facepiece respirator models," Journal of the International Society for Respiratory Protection, vol. 24, pp. 93-107, 2007.

[102] M. S. Bergman, D. J. Viscusi, B. K. Heimbuch, J. D. Wander, A. R. Sambol, and R. E. Shaffer, "Evaluation of multiple (3cycle) decontamination processing for filtering facepiece respirators," Journal of Engineered Fibers and Fabrics, vol. 5, no. 4, article 155892501000500405, 2018.

[103] M. S. Bergman, D. J. Viscusi, A. J. Palmiero, J. B. Powell, and R. E. Shaffer, "Impact of three cycles of decontamination treatments on filtering facepiece respirator fit," Journal of the International Society for Respiratory Protection, vol. 28, pp. 48-59, 2011.

[104] W. G. Lindsley, S. B. Martin Jr., R. E. Thewlis et al., "Effects of ultraviolet germicidal irradiation (UVGI) on N95 respirator filtration performance and structural integrity," Journal of Occupational and Environmental Hygiene, vol. 12, no. 8, pp. 509-517, 2015.

[105] L. Liao, W. Xiao, M. Zhao et al., "Can N95 respirators be reused after disinfection? How many times?," ACS Nano, vol. 14, no. 5, pp. 6348-6356, 2020.

[106] E. M. Fisher and R. E. Shaffer, "A method to determine the available UV-C dose for the decontamination of filtering facepiece respirators," Journal of Applied Microbiology, vol. 110, no. 1, pp. 287-295, 2011.

[107] B. K. Heimbuch, W. H. Wallace, K. Kinney et al., “A pandemic influenza preparedness study: use of energetic methods to decontaminate filtering facepiece respirators contaminated with H1N1 aerosols and droplets," American Journal of Infection Control, vol. 39, no. 1, pp. e1-e9, 2011.

[108] D. Mills, D. A. Harnish, C. Lawrence, M. Sandoval-Powers, and B. K. Heimbuch, "Ultraviolet germicidal irradiation of influenza-contaminated N95 filtering facepiece respirators," American Journal of Infection Control, vol. 46, no. 7, pp. e49-e55, 2018.

[109] R. Fischer, D. H. Morris, N. van Doremalen et al., Assessment of $\mathrm{N} 95$ respirator decontamination and re-use for SARS-CoV2, medRxiv, 2020.

[110] Q. Ou, C. Pei, S. C. Kim et al., “Original work: Covid-19 pandemic - decontamination of respirators and masks for the general.," https://www.apsf.org/article/covid-19-pandemicdecontamination-of-respirators-and-masks-for-the-generalpublic-healthcare-workers-and-hospital-environments/.

[111] K. Jansen, "During the coronavirus pandemic, hospitals have taken unprecedented steps to disinfect N95 face masks," in Chemical and Engineering News (cten), The American Chemical Society: Chemical and Engineering News, 2020.

[112] Final Report for the Bioquell Hydrogen Peroxide Vapor (HPV) Decontamination for Reuse of N95 Respirators, Battelle Columbus, OH, USA, 2016.

[113] A. Schwartz, M. Stiegel, N. Greeson et al., "Decontamination and reuse of N95 respirators with hydrogen peroxide vapor to address worldwide personal protective equipment shortages during the SARS-CoV-2 (COVID-19) pandemic," Applied Biosafety, vol. 25, no. 2, pp. 67-70, 2020.

[114] P. Kenney, B. K. Chan, K. Kortright et al., Hydrogen peroxide vapor sterilization of $\mathrm{N95}$ respirators for reuse, medRxiv, 2020. 
[115] A. Kumar, S. B. Kasloff, A. Leung et al., N95 mask decontamination using standard hospital sterilization technologies, medRxiv, 2020.

[116] J. S. Smith, H. Hanseler, J. Welle et al., Effect of various decontamination procedures on disposable N95 mask integrity and SARS-CoV-2 infectivity, medRxiv, 2020.

[117] K. Everington, "Rice cooker can be used to sterilize masks: Taiwanese study," https://www.taiwannews.com.tw/en/ news/3876567.

[118] D. F. Li, J. L. Cadnum, S. N. Redmond, L. D. Jones, and C. J. Donskey, "It's not the heat, it's the humidity: effectiveness of a rice cooker-steamer for decontamination of cloth and surgical face masks and N95 respirators," American Journal of Infection Control, vol. 48, no. 7, pp. 854855, 2020.

[119] E. Fisher, S. Rengasamy, D. Viscusi, E. Vo, and R. Shaffer, "Development of a test system to apply virus-containing particles to filtering facepiece respirators for the evaluation of decontamination procedures," Applied and Environmental Microbiology, vol. 75, no. 6, pp. 1500-1507, 2009.

[120] E. M. Fisher, J. L. Williams, and R. E. Shaffer, "Evaluation of microwave steam bags for the decontamination of filtering facepiece respirators," PLoS One, vol. 6, no. 4, article e18585, 2011.

[121] K. Pardee, A. A. Green, M. K. Takahashi et al., "Rapid, lowcost detection of zika virus using programmable biomolecular components," Cell, vol. 165, no. 5, pp. 1255-1266, 2016.

[122] Y. Xiang, Q. Song, and W. Gu, "Decontamination of surgical face masks and $\mathrm{N} 95$ respirators by dry heat pasteurization for one hour at $70^{\circ} \mathrm{C}$," American Journal of Infection Control, vol. 48, no. 8, pp. 880-882, 2020.

[123] B. K. Heimbuch, K. Kinney, A. E. Lumley, D. A. Harnish, M. Bergman, and J. D. Wander, "Cleaning of filtering facepiece respirators contaminated with mucin and _Staphylococcus aureus_," American Journal of Infection Control, vol. 42, no. 3, pp. 265-270, 2014.

[124] "Decontamination methods for $3 \mathrm{M}$ filtering facepiece respirators such as N95 respirators," https://multimedia.3m.com/ mws/media/18248690/decontamination-methods-for-3mfiltering-facepiece-respirators-technical-bulletin.pdf.

[125] F. E. Ahmed, B. S. Lalia, and R. Hashaikeh, "A review on electrospinning for membrane fabrication: challenges and applications," Desalination, vol. 356, pp. 15-30, 2015.

[126] C. Liu, P.-C. Hsu, H.-W. Lee et al., "Transparent air filter for high-efficiency $\mathrm{PM}_{2.5}$ capture," Nature Communications, vol. 6, no. 1, pp. 1-9, 2015.

[127] W. Liang, Y. Xu, X. Li et al., "Transparent polyurethane nanofiber air filter for high-efficiency $\mathrm{PM}_{2.5}$ capture," Nanoscale Research Letters, vol. 14, no. 1, p. 361, 2019.

[128] Q. Li, Y. Xu, H. Wei, and X. Wang, "An electrospun polycarbonate nanofibrous membrane for high efficiency particulate matter filtration," RSC Advances, vol. 6, no. 69, pp. 6527565281, 2016.

[129] Q. Zhang, Q. Li, T. M. Young, D. P. Harper, and S. Wang, "A novel method for fabricating an electrospun poly (vinyl alcohol)/cellulose nanocrystals composite nanofibrous filter with low air resistance for high-efficiency filtration of particulate matter," ACS Sustainable Chemistry \& Engineering, vol. 7, no. 9, pp. 8706-8714, 2019.

[130] H. Xu, W. Jin, F. Wang et al., "Preparation and properties of PTFE hollow fiber membranes for the removal of ultrafine particles in $\mathrm{PM}_{2.5}$ with repetitive usage capability," RSC Advances, vol. 8, no. 67, pp. 38245-38258, 2018.

[131] S. Lee, A. R. Cho, D. Park et al., "Reusable polybenzimidazole nanofiber membrane filter for highly breathable $\mathrm{PM}_{2.5}$ dust proof mask," ACS Applied Materials \& Interfaces, vol. 11, no. 3, pp. 2750-2757, 2018.

[132] S. Zhang, H. Liu, X. Yin, J. Yu, and B. Ding, “Anti-deformed polyacrylonitrile/polysulfone composite membrane with binary structures for effective air filtration," ACS Applied Materials \& Interfaces, vol. 8, no. 12, pp. 8086-8095, 2016.

[133] J. Liu, X. Zhang, H. Zhang et al., "Low resistance bicomponent spunbond materials for fresh air filtration with ultrahigh dust holding capacity," RSC Advances, vol. 7, no. 69, pp. 43879-43887, 2017.

[134] X. Yang, Y. Pu, Y. Zhang et al., "Multifunctional composite membrane based on $\mathrm{BaTiO}_{3} @ \mathrm{PU} / \mathrm{PSA}$ nanofibers for highefficiency PM2.5 removal," Journal of Hazardous Materials, vol. 391, p. 122254, 2020.

[135] J. Li, D. Zhang, T. Yang, S. Yang, X. Yang, and H. Zhu, "Nanofibrous membrane of graphene oxide-inpolyacrylonitrile composite with low filtration resistance for the effective capture of $\mathrm{PM}_{2.5}$., Journal of Membrane Science, vol. 551, pp. 85-92, 2018.

[136] X. Gao, Z.-K. Li, J. Xue et al., “Titanium carbide $\mathrm{Ti}_{3} \mathrm{C}_{2} \mathrm{~T}_{\mathrm{x}}$ (MXene) enhanced PAN nanofiber membrane for air purification," Journal of Membrane Science, vol. 586, pp. 162-169, 2019.

[137] L. Wang, C. Zhang, F. Gao, and G. Pan, "Needleless electrospinning for scaled-up production of ultrafine chitosan hybrid nanofibers used for air filtration," RSC Advances, vol. 6, no. 107, pp. 105988-105995, 2016.

[138] Z. Li, J. Song, Y. Long et al., "Large-scale blow spinning of heat-resistant nanofibrous air filters," Nano Research, vol. 13, no. 3, pp. 861-867, 2020.

[139] N. Wang, Y. Yang, S. S. Al-Deyab, M. El-Newehy, J. Yu, and B. Ding, "Ultra-light 3D nanofibre-nets binary structured nylon 6-polyacrylonitrile membranes for efficient filtration of fine particulate matter," Journal of Materials Chemistry A, vol. 3, no. 47, pp. 23946-23954, 2015.

[140] J. Liu, H. Zhang, H. Gong, X. Zhang, Y. Wang, and X. Jin, "Polyethylene/polypropylene bicomponent spunbond air filtration materials containing magnesium stearate for efficient fine particle capture," ACS Applied Materials \& Interfaces, vol. 11, no. 43, pp. 40592-40601, 2019.

[141] G. Q. Gu, C. B. Han, C. X. Lu et al., "Triboelectric nanogenerator enhanced nanofiber air filters for efficient particulate matter removal," ACS Nano, vol. 11, no. 6, pp. 6211-6217, 2017.

[142] G. Liu, J. Nie, C. Han et al., "Self-powered electrostatic adsorption face mask based on a triboelectric nanogenerator," ACS Applied Materials \& Interfaces, vol. 10, no. 8, pp. 7126-7133, 2018.

[143] X. Wang, B. Ding, G. Sun, M. Wang, and J. Yu, "Electro-spinning/netting: a strategy for the fabrication of threedimensional polymer nano-fiber/nets," Progress in Materials Science, vol. 58, no. 8, pp. 1173-1243, 2013.

[144] S. Zhang, N. Tang, L. Cao, X. Yin, J. Yu, and B. Ding, "Highly integrated polysulfone/polyacrylonitrile/polyamide-6 air filter for multilevel physical sieving airborne particles," ACS Applied Materials \& Interfaces, vol. 8, no. 42, pp. 2906229072, 2016. 
[145] X. Li, C. Wang, X. Huang et al., “Anionic surfactant-triggered steiner geometrical poly (vinylidene fluoride) nanofiber/nanonet air filter for efficient particulate matter removal," ACS Applied Materials \& Interfaces, vol. 10, no. 49, pp. 4289142904, 2018.

[146] P. P. Tsai, H. Schreuder-Gibson, and P. Gibson, "Different electrostatic methods for making electret filters," Journal of Electrostatics, vol. 54, no. 3-4, pp. 333-341, 2002.

[147] S. Wang, X. Zhao, X. Yin, J. Yu, and B. Ding, "Electret polyvinylidene fluoride nanofibers hybridized by polytetrafluoroethylene nanoparticles for high-efficiency air filtration," ACS Applied Materials \& Interfaces, vol. 8, no. 36, pp. 23985-23994, 2016.

[148] F. Liu, M. Li, W. Shao et al., "Preparation of a polyurethane electret nanofiber membrane and its air- filtration performance," Journal of Colloid and Interface Science, vol. 557, pp. 318-327, 2019.

[149] X. Ding, Y. Li, Y. Si, X. Yin, J. Yu, and B. Ding, "Electrospun polyvinylidene fluoride/ $\mathrm{SiO}_{2}$ nanofibrous membranes with enhanced electret property for efficient air filtration," Composites Communications, vol. 13, pp. 57-62, 2019.

[150] H. Zhang, J. Liu, X. Zhang, C. Huang, and X. Jin, "Design of electret polypropylene melt blown air filtration material containing nucleating agent for effective PM2. 5 capture," RSC Advances, vol. 8, no. 15, pp. 7932-7941, 2018.

[151] P. Jiang, X. Zhao, Y. Li et al., "Moisture and oily molecules stable nanofibrous electret membranes for effectively capturing $\mathrm{PM}_{2.5}$," Composites Communications, vol. 6, pp. 34-40, 2017.

[152] X. Wang, S. Niu, F. Yi et al., "Harvesting ambient vibration energy over a wide frequency range for self-powered electronics," ACS Nano, vol. 11, no. 2, pp. 1728-1735, 2017.

[153] Y. C. Lai, J. Deng, S. L. Zhang, S. Niu, H. Guo, and Z. L. Wang, "Single-thread-based wearable and highly stretchable triboelectric nanogenerators and their applications in clothbased self-powered human-interactive and biomedical sensing," Advanced Functional Materials, vol. 27, no. 1, p. 1604462, 2017.

[154] Y. Bai, C. B. Han, C. He et al., "Washable multilayer triboelectric air filter for efficient particulate matter $\mathrm{PM}_{2.5}$ removal," Advanced Functional Materials, vol. 28, no. 15, article 1706680, 2018.

[155] S. Qiu, M. Xue, and G. Zhu, "Metal-organic framework membranes: from synthesis to separation application," Chemical Society Reviews, vol. 43, no. 16, pp. 6116-6140, 2014.

[156] Y. Chen, S. Zhang, S. Cao et al., "Roll-to-roll production of metal-organic framework coatings for particulate matter removal," Advanced Materials, vol. 29, no. 15, article $1606221,2017$.

[157] W.-T. Koo, J.-S. Jang, S. Qiao et al., "Hierarchical metalorganic framework-assembled membrane filter for efficient removal of particulate matter," ACS Applied Materials \& Interfaces, vol. 10, no. 23, pp. 19957-19963, 2018.

[158] Z. Hao, J. Wu, C. Wang, and J. Liu, "Electrospun polyimide/metal-organic framework nanofibrous membrane with superior thermal stability for efficient $\mathrm{PM}_{2.5}$ capture," ACS Applied Materials \& Interfaces, vol. 11, no. 12, pp. 1190411909, 2019.

[159] Y. Zhang, S. Yuan, X. Feng, H. Li, J. Zhou, and B. Wang, "Preparation of nanofibrous metal-organic framework filters for efficient air pollution control," Journal of the American Chemical Society, vol. 138, no. 18, pp. 5785-5788, 2016.

[160] A. A. I. A. S. Komaladewi, K. Khoiruddin, I. W. Surata, I. D. G. A. Subagia, and I. G. Wenten, "Recent advances in antimicrobial air filter," E3S Web of Conferences, vol. 67, article 03016, 2018.

[161] D. H. Lee, J. H. Jung, and B. U. Lee, "Effect of treatment with a natural extract of Mukdenia Rossii (Oliv) Koidz and unipolar ion emission on the antibacterial performance of air filters," Aerosol and Air Quality Research, vol. 13, no. 2, pp. 771776, 2013.

[162] C. G. Woo, H.-J. Kim, Y.-J. Kim, and B. Han, "Enhanced antimicrobial activity on non-conducting and conducting air filters by using air ions and grapefruit seed extract," Aerosol and Air Quality Research, vol. 17, no. 7, pp. 1917-1924, 2017.

[163] G. B. Hwang, K. M. Sim, G.-N. Bae, and J. H. Jung, "Synthesis of hybrid carbon nanotube structures coated with Sophora flavescens nanoparticles and their application to antimicrobial air filtration," Journal of Aerosol Science, vol. 86, pp. 44-54, 2015.

[164] G. B. Hwang, K. J. Heo, J. H. Yun et al., “Antimicrobial air filters using natural euscaphis japonica nanoparticles," PLoS One, vol. 10, no. 5, article e0126481, 2015.

[165] C. G. Woo, J.-S. Kang, H.-J. Kim, Y.-J. Kim, and B. Han, "Treatment of air filters using the antimicrobial natural products propolis and grapefruit seed extract for deactivation of bioaerosols," Aerosol Science and Technology, vol. 49, no. 8, pp. 611-619, 2015.

[166] R. Huang, O. V. Pyankov, B. Yu, and I. E. Agranovski, "Inactivation of fungal spores collected on fibrous filters by Melaleuca alternifolia (tea tree oil)," Aerosol Science and Technology, vol. 44, no. 4, pp. 262-268, 2010.

[167] P. Ekabutr, P. Chuysinuan, S. Suksamrarn, W. Sukhumsirichart, P. Hongmanee, and P. Supaphol, "Development of antituberculosis melt-blown polypropylene filters coated with mangosteen extracts for medical face mask applications," Polymer Bulletin, vol. 76, no. 4, pp. 1985-2004, 2019.

[168] E.-S. Chong, G. B. Hwang, C. W. Nho et al., "Antimicrobial durability of air filters coated with airborne Sophora flavescens nanoparticles," Science of the Total Environment, vol. 444, pp. 110-114, 2013.

[169] G. B. Hwang, J. E. Lee, C. W. Nho et al., "Short-term effect of humid airflow on antimicrobial air filters using Sophora flavescens nanoparticles," Science of the Total Environment, vol. 421-422, pp. 273-279, 2012.

[170] K. M. Sim, H. J. Lee, C. W. Nho, G.-N. Bae, and J. H. Jung, "Effects of surrounding temperature on antimicrobial air filters coated with Sophora flavescens nanoparticles," Aerosol Science and Technology, vol. 48, no. 3, pp. 324-332, 2014.

[171] K. M. Sim, K. H. Kim, G. B. Hwang, S. Seo, G.-N. Bae, and J. H. Jung, "Development and evaluation of antimicrobial activated carbon fiber filters using Sophora flavescens nanoparticles," Science of the Total Environment, vol. 493, pp. 291-297, 2014.

[172] B. C. Son, C. H. Park, and C. S. Kim, "Fabrication of antimicrobial nanofiber air filter using activated carbon and cinnamon essential oil," Journal of Nanoscience and Nanotechnology, vol. 20, no. 7, pp. 4376-4380, 2020.

[173] J. Choi, B. J. Yang, G.-N. Bae, and J. H. Jung, "Herbal extract incorporated nanofiber fabricated by an electrospinning 
technique and its application to antimicrobial air filtration," ACS Applied Materials \& Interfaces, vol. 7, no. 45, pp. 25313-25320, 2015.

[174] A. Baranwal, A. Srivastava, P. Kumar, V. K. Bajpai, P. K. Maurya, and P. Chandra, "Prospects of nanostructure materials and their composites as antimicrobial agents," Frontiers in Microbiology, vol. 9, no. 422, 2018.

[175] N. Beyth, Y. Houri-Haddad, A. Domb, W. Khan, and R. Hazan, "Alternative antimicrobial approach: nanoantimicrobial materials," Evidence-Based Complementary and Alternative Medicine, vol. 2015, Article ID 246012, 16 pages, 2015.

[176] R. Lakshminarayanan, E. Ye, D. J. Young, Z. Li, and X. J. Loh, "Recent advances in the development of antimicrobial nanoparticles for combating resistant pathogens," Advanced Healthcare Materials, vol. 7, no. 13, article e1701400, 2018.

[177] V. Binas, D. Venieri, D. Kotzias, and G. Kiriakidis, "Modified $\mathrm{TiO}_{2}$ based photocatalysts for improved air and health quality," Journal of Materiomics, vol. 3, no. 1, pp. 3-16, 2017.

[178] J. H. Jung, G. B. Hwang, J. E. Lee, and G. N. Bae, "Preparation of airborne Ag/CNT hybrid nanoparticles using an aerosol process and their application to antimicrobial air filtration," Langmuir, vol. 27, no. 16, pp. 10256-10264, 2011.

[179] L. Pei, J. Zhou, and L. Zhang, "Preparation and properties of Ag-coated activated carbon nanocomposites for indoor air quality control," Building and Environment, vol. 63, pp. 108-113, 2013.

[180] J.-W. Chen, G. W.-M. Lee, K.-J. Chen, and S.-H. Yang, "Control of bioaerosols in indoor environment by filter coated with nanosilicate platelet supported silver nanohybrid (AgNPs/NSP)," Aerosol and Air Quality Research, vol. 16, no. 9, pp. 2198-2207, 2016.

[181] Y. Li, P. Leung, L. Yao, Q. W. Song, and E. Newton, “Antimicrobial effect of surgical masks coated with nanoparticles," The Journal of Hospital Infection, vol. 62, no. 1, pp. 58-63, 2006.

[182] P. Chuaybamroong, R. Chotigawin, S. Supothina, P. Sribenjalux, S. Larpkiattaworn, and C.-Y. Wu, "Efficacy of photocatalytic HEPA filter on microorganism removal," Indoor Air, vol. 20, no. 3, pp. 246-254, 2010.

[183] Z. Wang, Z. Pan, J. Wang, and R. Zhao, "A novel hierarchical structured poly(lactic acid)/titania fibrous membrane with excellent antibacterial activity and air filtration performance," Journal of Nanomaterials, vol. 2016, Article ID 6272983, 17 pages, 2016.

[184] J. Hu, Z. Zhong, F. Zhang, W. Xing, Z.-X. Low, and Y. Fan, "Coating of $\mathrm{ZnO}$ nanoparticles onto the inner pore channel surface of $\mathrm{SiC}$ foam to fabricate a novel antibacterial air filter material," Ceramics International, vol. 41, no. 5, pp. 70807090, 2015.

[185] Z. Zhong, Z. Xu, T. Sheng, J. Yao, W. Xing, and Y. Wang, "Unusual air filters with ultrahigh efficiency and antibacterial functionality enabled by $\mathrm{ZnO}$ nanorods," ACS Applied Materials \& Interfaces, vol. 7, no. 38, pp. 21538-21544, 2015.

[186] A. A. I. Agung Sri Komaladewi, K. Khoiruddin, I. Dewa Gede Ary Subagia, D. I. Astuti, and I. Gede Wenten, "Antimicrobial hollow fiber polypropylene/ZnO membrane for effective air filtration," IOP Conference Series: Materials Science and Engineering, vol. 622, article 012005, 2019.

[187] D. Y. Choi, K. J. Heo, J. Kang et al., "Washable antimicrobial polyester/aluminum air filter with a high capture efficiency and low pressure drop," Journal of Hazardous Materials, vol. 351, pp. 29-37, 2018.

[188] S. Feng, D. Li, Z. X. Low et al., “ALD-seeded hydrothermallygrown $\mathrm{Ag} / \mathrm{ZnO}$ nanorod PTFE membrane as efficient indoor air filter," Journal of Membrane Science, vol. 531, pp. 86-93, 2017.

[189] Y. Zhao, Z.-X. Low, S. Feng, Z. Zhong, Y. Wang, and Z. Yao, "Multifunctional hybrid porous filters with hierarchical structures for simultaneous removal of indoor VOCs, dusts and microorganisms," Nanoscale, vol. 9, no. 17, pp. 54335444, 2017.

[190] L. Tamayo, M. Azócar, M. Kogan, A. Riveros, and M. Páez, "Copper-polymer nanocomposites: an excellent and cost-effective biocide for use on antibacterial surfaces," Materials Science and Engineering: C, vol. 69, pp. 13911409, 2016.

[191] M. Shen, F. Forghani, X. Kong et al., "Antibacterial applications of metal-organic frameworks and their composites," Comprehensive Reviews in Food Science and Food Safety, vol. 19, no. 4, pp. 1397-1419, 2020.

[192] N. Bhardwaj, S. K. Pandey, J. Mehta, S. K. Bhardwaj, K.H. Kim, and A. Deep, "Bioactive nano-metal-organic frameworks as antimicrobials against Gram-positive and Gramnegative bacteria," Toxicology Research, vol. 7, no. 5, pp. 931-941, 2018.

[193] S. K. Springthorpe, C. M. Dundas, and B. K. Keitz, "Microbial reduction of metal-organic frameworks enables synergistic chromium removal," Nature Communications, vol. 10, no. 1, p. 5212, 2019.

[194] G. Wyszogrodzka, B. Marszałek, B. Gil, and P. Dorożyński, "Metal-organic frameworks: mechanisms of antibacterial action and potential applications," Drug Discovery Today, vol. 21, no. 6, pp. 1009-1018, 2016.

[195] S. Ma, M. Zhang, J. Nie, B. Yang, S. Song, and P. Lu, "Multifunctional cellulose-based air filters with high loadings of metal-organic frameworks prepared by in situ growth method for gas adsorption and antibacterial applications," Cellulose, vol. 25, no. 10, pp. 5999-6010, 2018.

[196] P. Li, J. Li, X. Feng et al., "Metal-organic frameworks with photocatalytic bactericidal activity for integrated air cleaning," Nature Communications, vol. 10, no. 1, p. 2177, 2019.

[197] A. W. H. Chin, J. T. S. Chu, M. R. A. Perera et al., "Stability of SARS-CoV-2 in different environmental conditions," The Lancet Microbe, vol. 1, no. 1, article e10, 2020.

[198] B. Bean, B. M. Moore, B. Sterner, L. R. Peterson, D. N. Gerding, and H. H. Balfour Jr., "Survival of influenza viruses on environmental surfaces," The Journal of Infectious Diseases, vol. 146, no. 1, pp. 47-51, 1982.

[199] M. M. Weiss, P. D. Weiss, D. E. Weiss, and J. B. Weiss, "Disrupting the transmission of influenza a: face masks and ultraviolet light as control measures," American Journal of Public Health, vol. 97, Supplement 1, pp. S32-S37, 2007.

[200] G. Kampf, A. Voss, and S. Scheithauer, "Inactivation of coronaviruses by heat," Journal of Hospital Infection, vol. 105, no. 2, pp. 348-349, 2020.

[201] “Administration, U. S. F. D. Product classification,” May, https://www.accessdata.fda.gov/scripts/cdrh/cfdocs/cfPCD/ classification.cfm.

[202] “Administration, U. S. F. D. 510(k) premarket notification," May, https://www.accessdata.fda.gov/scripts/cdrh/cfdocs/ cfpmn/pmn.cfm. 
[203] Y. N. Slavin, J. Asnis, U. O. Häfeli, and H. Bach, "Metal nanoparticles: understanding the mechanisms behind antibacterial activity," Journal of Nanobiotechnology, vol. 15, no. 1, p. $65,2017$.

[204] A. J. Huh and Y. J. Kwon, “"Nanoantibiotics": a new paradigm for treating infectious diseases using nanomaterials in the antibiotics resistant era," Journal of Controlled Release, vol. 156, no. 2, pp. 128-145, 2011.

[205] E. Sánchez-López, D. Gomes, G. Esteruelas et al., "Metalbased nanoparticles as antimicrobial agents: an overview," Nanomaterials, vol. 10, no. 2, p. 292, 2020.

[206] S. M. Dizaj, F. Lotfipour, M. Barzegar-Jalali, M. H. Zarrintan, and K. Adibkia, "Antimicrobial activity of the metals and metal oxide nanoparticles," Materials Science and Engineering C, vol. 44, pp. 278-284, 2014.

[207] E. Hoseinzadeh, P. Makhdoumi, P. Taha et al., "A review on nano-antimicrobials: metal nanoparticles, methods and mechanisms," Current Drug Metabolism, vol. 18, no. 2, pp. 120-128, 2017.

[208] G. R. Rudramurthy, M. K. Swamy, U. R. Sinniah, and A. Ghasemzadeh, "Nanoparticles: alternatives against drugresistant pathogenic microbes," Molecules, vol. 21, no. 7, p. 836, 2016.

[209] N.-Y. Lee, W.-C. Ko, and P.-R. Hsueh, "Nanoparticles in the treatment of infections caused by multidrug-resistant organisms," Frontiers in Pharmacology, vol. 10, p. 1153, 2019.

[210] N. Durán, M. Durán, M. B. de Jesus, A. B. Seabra, W. J. Fávaro, and G. Nakazato, "Silver nanoparticles: a new view on mechanistic aspects on antimicrobial activity," Nanomedicine: Nanotechnology, Biology, and Medicine, vol. 12, no. 3, pp. 789-799, 2016.

[211] M. K. Rai, S. D. Deshmukh, A. P. Ingle, and A. K. Gade, "Silver nanoparticles: the powerful nanoweapon against multidrug-resistant bacteria," Journal of Applied Microbiology, vol. 112, no. 5, pp. 841-852, 2012.

[212] A. C. Burdușel, O. Gherasim, A. M. Grumezescu, L. Mogoantă, A. Ficai, and E. Andronescu, "Biomedical applications of silver nanoparticles: an up-to-date overview," Nanomaterials, vol. 8, no. 9, p. 681, 2018.

[213] J. Yan and J. Cheng, "Antimicrobial yarn having nanosilver particles and methods for manufacturing the same," US Patent 6979491, 2005.

[214] J. Zhou and T. Shi, "Prevent antibiotic protective facial mask of haze humidification," US Patent 206565343U, 2017.

[215] G. Ren, J. S. Oxford, P. W. Reip, R. Lambkin-Willams, and A. Mann, "Anti-v0iral formulations nanomaterials and nanoparticles," US Patent 20100040655A1, 2010.

[216] C. Wang, J. Li, G. Yang, and J. Zhou, "Protective facial mask containing nano silver and chitosan and production method of protective facial mask," US Patent 102144817, 2011.

[217] D. Kharaghani, M. Q. Khan, A. Shahrzad et al., "Preparation and in-vitro assessment of hierarchal organized antibacterial breath mask based on polyacrylonitrile/silver (PAN/AgNPs) nanofiber," Nanomaterials, vol. 8, no. 7, p. 461, 2018.

[218] C. B. Hiragond, A. S. Kshirsagar, V. V. Dhapte, T. Khanna, P. Joshi, and P. V. More, "Enhanced anti-microbial response of commercial face mask using colloidal silver nanoparticles," Vacuum, vol. 156, pp. 475-482, 2018.

[219] G. Ren, D. Hu, E. W. C. Cheng, M. A. Vargas-Reus, P. Reip, and R. P. Allaker, "Characterisation of copper oxide nanopar- ticles for antimicrobial applications," International Journal of Antimicrobial Agents, vol. 33, no. 6, pp. 587-590, 2009.

[220] L. P. Arendsen, R. Thakar, and A. H. Sultan, "The use of copper as an antimicrobial agent in health care, including obstetrics and gynecology," Clinical Microbiology Reviews, vol. 32, no. 4, 2019 .

[221] G. Borkow and J. Gabbay, "Copper, an ancient remedy returning to fight microbial, fungal and viral infections," Current Chemical Biology, vol. 3, pp. 272-278, 2012.

[222] G. Borkow and J. Gabbay, "Copper as a biocidal tool," Current Medicinal Chemistry, vol. 12, no. 18, pp. 2163-2175, 2005.

[223] S. S. Goh, S. Guduguntla, T. Kikuchi et al., "Desymmetrization of meso-dibromocycloalkenes through copper(I)-catalyzed asymmetric allylic substitution with organolithium reagents," Journal of the American Chemical Society, vol. 140, no. 23, pp. 7052-7055, 2018.

[224] G. Borkow, S. S. Zhou, T. Page, and J. Gabbay, "A novel antiinfluenza copper oxide containing respiratory face mask," PLoS One, vol. 5, no. 6, p. e11295, 2010.

[225] F. Yoshie, N. Tsuruo, and S. Tetsuya, "Antiviral agent," US Patent 5746406, 2015.

[226] NBC Meshtec Inc, 510(k) Summary K182766 Cufitec Surgical Mask, 2019.

[227] J. Gabbay, "Antimicrobial and antiviral polymeric materials," US Patent 7169402, 2007.

[228] J. Gabbay, "Antimicrobial, antifungal and antiviral rayon fibers," US Patent 8741197, 2014.

[229] J. Gabbay, "Antimicrobial and antiviral polymeric master batch, processes for producing polymeric material therefrom and products produced therefrom," US Patent 101184397, 2011.

[230] J. W. Kim, “Multi-layer knitted structure fabric with air layer using conductive copper material and integrated antibacterial mask using thereof," US Patent 102000730B1, 2019.

[231] J. W. Kim, "Reusable antibacterial maks for fine dust," US Patent 102094480B1, 2020.

[232] H. Zhong, Z. Zhu, J. Lin et al., "Reusable and recyclable graphene masks with outstanding superhydrophobic and photothermal performances," ACS Nano, vol. 14, no. 5, pp. 62136221, 2020.

[233] D. Xu, X. Liang, R. Donnelly, and N. Cranstoun, "Fabric," US Patent 2527849A, 2016.

[234] Copper Clothing Ltd, “Antimicrobial material," US Patent 201819857D0, 2019.

[235] W. Wang, G. Huang, J. C. Yu, and P. K. Wong, "Advances in photocatalytic disinfection of bacteria: development of photocatalysts and mechanisms," Journal of Environmental Sciences (China), vol. 34, pp. 232-247, 2015.

[236] J. Bogdan, J. Zarzyńska, and J. Pławińska-Czarnak, “Comparison of infectious agents susceptibility to photocatalytic effects of nanosized titanium and zinc oxides: a practical approach," Nanoscale Research Letters, vol. 10, pp. 309-323, 2011.

[237] P. Ganguly, C. Byrne, A. Breen, and S. C. Pillai, "Antimicrobial activity of photocatalysts: fundamentals, mechanisms, kinetics and recent advances," Applied Catalysis B: Environmental, vol. 225, pp. 51-75, 2018.

[238] K. Takashi, K. Akira, and Y. Akihiko, "Infection prevention mask," US Patent 2005124777A, 2005. 
[239] W. X. Lee and M. Nu, "Functional protective mask," US Patent 201320356118, 2013.

[240] A. Gedanken, Y. Nitzan, I. Perelshtein, N. Perkas, and G. Applerot, "Sonochemical coating of textiles with metal oxide nanoparticles for antimicrobial fabrics," US Patent 9315937, 2009.

[241] A. Gedanken, Y. Nitzan, I. Perelshtein, N. Perkas, and G. Applerot, "Sonochemical coating of textiles with metal oxide nanoparticles for antimicrobial fabrics," US Patent 10370789, 2019.

[242] A. Panáček, L. Kvítek, M. Smékalová et al., "Bacterial resistance to silver nanoparticles and how to overcome it," Nature Nanotechnology, vol. 13, no. 1, pp. 65-71, 2018.

[243] J. L. Hobman and L. C. Crossman, "Bacterial antimicrobial metal ion resistance," Journal of Medical Microbiology, vol. 64, no. 5, pp. 471-497, 2015.

[244] M. Kanovsky, "Antimicrobial fabric materials for use in safety masks and personal protection clothing," US Patent WO2016125173A1, 2016.

[245] M. Kanovsky, "Antimicrobial material comprising synergistic combinations of metal oxides," US Patent 20200100503A1, 2020.

[246] M. Nishide, K. Tsujimoto, M. Uozaki et al., "Effects of electrolytes on virus inactivation by acidic solutions," International Journal of Molecular Medicine, vol. 27, no. 6, pp. 803-809, 2011.

[247] M. E. Lombardi, B. S. Ladman, R. L. Alphin, and E. R. Benson, "Inactivation of avian influenza virus using common detergents and chemicals," Avian Diseases, vol. 52, no. 1, pp. 118-123, 2008.

[248] J. H. Lemelson, "Face mask and method," US Patent 4856509 , 1989.

[249] GlaxoSmithKline, 510(k) Summary K081923 Actiprotect UF N95 Respirator, 2009.

[250] GlaxoSmithKline, 510(k) Summary K093161 Actiprotect UF N95 Respirator, 2010.

[251] F. S. Quan, I. Rubino, S. H. Lee, B. Koch, and H. J. Choi, "Universal and reusable virus deactivation system for respiratory protection," Scientific Reports, vol. 7, no. 1, pp. 1-10, 2017.

[252] J. M. Song, K. H. Lee, and B. L. Seong, "Antiviral effect of catechins in green tea on influenza virus," Antiviral Research, vol. 68, no. 2, pp. 66-74, 2005.

[253] R. Bahramsoltani, H. R. Sodagari, M. H. Farzaei, A. H. Abdolghaffari, M. Gooshe, and N. Rezaei, "The preventive and therapeutic potential of natural polyphenols on influenza," Expert Review of Anti-Infective Therapy, vol. 14, no. 1, pp. 57-80, 2015.

[254] K. Nashimoto and Y. Tashiro, "Gargling cup, antiviral mask, antiviral filter, antifungal, antibacterial, and antiviral filter air cleaner and air-cleaner humidifier," US Patent 5888527, 1999.

[255] M. Catel-Ferreira, H. Tnani, C. Hellio, P. Cosette, and L. Lebrun, "Antiviral effects of polyphenols: development of bio-based cleaning wipes and filters," Journal of Virological Methods, vol. 212, pp. 1-7, 2015.

[256] E. A. Abbott and A. J. Isquith, "Method of inhibiting the growth of bacteria and fungi using organosilicon amines," US Patent 3794736, 1974.

[257] A. J. Isquith, E. A. Abbott, and P. A. Walters, "Surfacebonded antimicrobial activity of an organosilicon quaternary ammonium chloride," Applied Microbiology, vol. 24, no. 6, pp. 859-863, 1972.

[258] A. J. Isquith and C. J. McCollum, "Surface kinetic test method for determining rate of kill by an antimicrobial solid," Applied and Environmental Microbiology, vol. 36, no. 5, pp. 700-704, 1978.

[259] A. M. Puckett and M. G. Mitchell, "Transparent antimicrobial face mask," US Patent US20190069615A1, 2010.

[260] M. P. Mathis, U. W. J. Bowne, E. C. Steindorf et al., "Germicidal face mask," US Patent 7845351, 2010.

[261] G. Tiliket, D. L. Sage, V. Moules et al., "A new material for airborne virus filtration," Chemical Engineering Journal, vol. 173, no. 2, pp. 341-351, 2011.

[262] K. Biedermann, F. Deng, S. King, and A. Middleton, "Antiviral mask and filter material," US Patent WO2008009651A1, 2008.

[263] W. Sun and F. G. Wu, "Two-dimensional materials for antimicrobial applications: graphene materials and beyond," Chemistry - An Asian Journal, vol. 13, no. 22, pp. 33783410, 2018.

[264] Y. L. F. Musico, C. M. Santos, M. L. P. Dalida, and D. F. Rodrigues, "Surface modification of membrane filters using graphene and graphene oxide-based nanomaterials for bacterial inactivation and removal," ACS Sustainable Chemistry \& Engineering, vol. 2, no. 7, pp. 1559-1565, 2014.

[265] J. Zhu, J. Wang, J. Hou, Y. Zhang, J. Liu, and B. Van der Bruggen, "Graphene-based antimicrobial polymeric membranes: a review," Journal of Materials Chemistry A, vol. 5, no. 15, pp. 6776-6793, 2017.

[266] M. G. Stanford, J. T. Li, Y. Chen et al., "Self-sterilizing laserinduced graphene bacterial air filter," ACS Nano, vol. 13, no. 10, pp. 11912-11920, 2019.

[267] K. Turcheniuk, C.-H. Hage, J. Spadavecchia et al., "Plasmonic photothermal destruction of uropathogenic E. coli with reduced graphene oxide and core/shell nanocomposites of gold nanorods/reduced graphene oxide," Journal of Materials Chemistry B, vol. 3, no. 3, pp. 375-386, 2015.

[268] L. Hui, J. T. Auletta, Z. Huang et al., "Surface disinfection enabled by a layer-by-layer thin film of polyelectrolytestabilized reduced graphene oxide upon solar near-infrared irradiation," ACS Applied Materials \& Interfaces, vol. 7, no. 19, pp. 10511-10517, 2015.

[269] C. Liu, D. Kong, P.-C. Hsu et al., "Rapid water disinfection using vertically aligned $\mathrm{MoS}_{2}$ nanofilms and visible light," Nature Nanotechnology, vol. 11, no. 12, pp. 1098-1104, 2016.

[270] S. Pandit, S. Karunakaran, S. K. Boda, B. Basu, and M. De, "High antibacterial activity of functionalized chemically exfoliated $\mathrm{MoS}_{2}$," ACS Applied Materials \& Interfaces, vol. 8, no. 46, pp. 31567-31573, 2016.

[271] X. Yang, J. Li, T. Liang et al., "Antibacterial activity of twodimensional $\mathrm{MoS}_{2}$ sheets," Nanoscale, vol. 6, no. 17, pp. 10126-10133, 2014.

[272] F. Alimohammadi, M. Sharifian Gh, N. H. Attanayake et al., "Antimicrobial properties of $2 \mathrm{D} \mathrm{MnO}_{2}$ and $\mathrm{MoS}_{2}$ nanomaterials vertically aligned on graphene materials and $\mathrm{Ti}_{3} \mathrm{C}_{2}$ MXene," Langmuir, vol. 34, no. 24, pp. 7192-7200, 2018.

[273] J. W. Cherrie, S. Wang, W. Mueller, C. Wendelboe-Nelson, and M. Loh, "In-mask temperature and humidity can validate respirator wear-time and indicate lung health status," Journal of Exposure Science \& Environmental Epidemiology, vol. 29, no. 4, pp. 578-583, 2019. 
[274] L. Sun, T. Du, C. Hu et al., "Antibacterial activity of graphene oxide/g- $\mathrm{C}_{3} \mathrm{~N}_{4}$ composite through photocatalytic disinfection under visible light," ACS Sustainable Chemistry \& Engineering, vol. 5, no. 10, pp. 8693-8701, 2017.

[275] J. H. Thurston, N. M. Hunter, and K. A. Cornell, "Preparation and characterization of photoactive antimicrobial graphitic carbon nitride $\left(\mathrm{g}-\mathrm{C}_{3} \mathrm{~N}_{4}\right)$ films," RSC Advances, vol. 6, no. 48, pp. 42240-42248, 2016.

[276] A. Smith and J. Taylor, "Antiviral metal impregnated activated carbon cloth components," US Patent 20110114095A1, 2011.

[277] A. M. Davison, "Pathogen inactivation and filtration efficacy of a new anti-microbial and anti-viral surgical facemask and N95 against dentistry-associated microorganisms," International Dentistry-Australasian Edition, vol. 7, pp. 36-42, 2012.

[278] Filigent (HK) Limited, 510(k) Summary K122702 BioFriend Biomask Surgical Mask, 2011.

[279] Filigent (HK) Limited, 510(k) Summary K122702 BioFriend Biomask N95 Surgical Respirator, 2013.

[280] Innonix Technologies Limited, 510(k) Summary K192105 Innonix Antiviral Child Mask, 2019.

[281] N. G. Stewart, F. C. N. Lau, T. W. Kung, L. Y. Lo, D. J. Ryan, and R. W. von Borstel, "Composition for use in decreasing the transmission of human pathogens," US Patent 20120060258A1, 2012.

[282] N. G. Stewart, L. Y. Lo, F. C. N. Lau, D. J. Ryan, and R. W. von Borstel, "Devices and methods for decreasing human pathogen transmission," US Patent 8678002, 2014.

[283] Nexera Medical, 510(k) Summary K120244 SpectraShield model 9500 Surgical Mask, 2012.

[284] J. A. Trogolo J. E. Barry et al., US Patent 6436422, 2002.

[285] J. A. Trogolo, E. A. P. Johnston, E. Pervin, A. Stahl, and M. Hyman, "Bi-laminar, hyaluronan coatings with silverbased anti-microbial properties," US Patent 6866859, 2005.

[286] S. Foss, "Anti-microbial fiber and fibrous products," US Patent 6946196, 2005.

[287] Y. Imashiro, N. Sasaki, Y. Ogushi, and M. Iizuka, "Antimicrobial, dustproof fabric and mask," US Patent 20110232653A1, 2011.

[288] “Anson nano clinical report | ANSON nano," May, http:// www.ansonnano.cn/xinyemian14/detail_40341.

[289] “Copperline Australia test results," May, https://www .copperlineaustralia.com/pages/testing-standards-as-of-2803-2020.

[290] "Aljazeera.com, Which countries have made wearing face masks compulsory?," 2020.

[291] World Health Organisation, Deafness, 2020.

[292] H. McGurk and J. Macdonald, "Hearing lips and seeing voices," Nature, vol. 264, no. 5588, pp. 746-748, 1976.

[293] M. W. Schurgin, J. Nelson, S. Iida, H. Ohira, J. Y. Chiao, and S. L. Franconeri, "Eye movements during emotion recognition in faces," Journal of Vision, vol. 14, no. 13, pp. 14-14, 2014.

[294] A. Mehrabian and S. R. Ferris, "Inference of attitudes from nonverbal communication in two channels," Journal of Consulting Psychology, vol. 31, no. 3, pp. 248-252, 1967.

[295] "Reuters, China firm develops system to recognize faces behind coronavirus masks," 2020.

[296] A. K. Leichman, "A face-recognition tech that works even for masked faces," https://www.israel21c.org/a-face-recognitiontech-that-works-even-for-masked-faces/.
[297] “Sanitary mask. CN107073303B,” 2020.

[298] M. Kakinuma, S. Ku, M. Takenouchi et al., Transparent Laminate, 2018.

[299] J. Hahne, “Face mask," US Patent 2008/0092909A1, 2008.

[300] I. T. W. Lam and A. Hsu, "Face mask having transparent plastic piece,” US Patent US20190069615A1, 2018.

[301] Z. Lin and C. Xu, "Mask with transparent plastic sheet," US PAtent CN201880055529.XA, 2020.

[302] J. H. Steinberg, “Transparent odor-free face mask," US PAtent US4467799A, 1984.

[303] K.-S. Shim, "Sanitary mask for the protection of others," US PAtent US8375949B2, 2013.

[304] J. Howard, A. Huang, Z. Li et al., Face masks against COVID19: an evidence review, Preprints.org, 2020.

[305] C.-F. Kuo, "Mask that providesa comfortable sensation to a user," US Patent US20110296584A1, 2011.

[306] B. J. Huddart, D. A. Purnomo, K. B. Powell, B. Patel, and E. L. Duckworth, "Breathable respiratory mask," US Patent US20050056286, 2004.

[307] K. Yang, J. E. Fish, O. P. Thomas, and M. S. Shamis, "Breathable protective articles," US Patent US20060143767, 2006.

[308] E. C. Steindorf, "Face mask having baffle layer for improved fluid resistance," US Patent 2005/0133036A1, 2003.

[309] L. C. Wadsworth and P. P.-Y. Tsai, "Enhancement of barrier fabrics with breathable films and of face masks and filters with novel fluorochemical electret reinforcing treatment," US Patent 2005/0079379A1, 2004.

[310] K. Wu and W. O. Mask, US Patent 2020/042096A1, 2018.

[311] V. Bansal, "Respiratory mask with microporous membrane and activated carbon," US Patent 2009/0211581A1, 2009.

[312] S. P. Prasad, C. K. Nash, E. C. Steindorf, A. Y. Houde, and J. D. Hurdler, "Face mask having improved comfort through cooling of microclimate through use of a phase change material," US Patent WO2017184798A1, 2017.

[313] Y. C. Lin and C. P. Chen, "Thermoregulation and thermal sensation in response to wearing tight-fitting respirators and exercising in hot-and-humid indoor environment," Building and Environment, vol. 160, article 106158, 2019.

[314] W. L. Yip, L. P. Leung, P. F. Lau, and H. K. Tong, "The effect of wearing a face mask on body temperature," Hong Kong Journal of Emergency Medicine, vol. 12, no. 1, pp. 23-27, 2017.

[315] "Innosparks - AIR+ mask," https://www.airplus-family.com/.

[316] N. Mistry, "Face mask designs for a surreal future where wearing masks is humanity's new norm | Yanko Design," https://www.yankodesign.com/2020/04/12/face-maskdesigns-for-a-surreal-future-where-wearing-masks-ishumanitys-new-norm/.

[317] C. J. Armusch, S. P. Singh, F. Sargunaraj, Y. Oren, J. M. Tour, and Y. Li, "Antibiofilm and antimicrobial functional membrane spacer," US Patent US20190143275A1, 2019.

[318] S. P. Singh, Y. Li, A. Be'er, Y. Oren, J. M. Tour, and C. J. Arnusch, "Laser-induced graphene layers and electrodes prevents microbial fouling and exerts antimicrobial action," ACS Applied Materials \& Interfaces, vol. 9, no. 21, pp. 1823818247, 2017.

[319] A. K. Thakur, S. P. Singh, M. N. Kleinberg, A. Gupta, and C. J. Arnusch, "Laser-induced graphene-PVA composites as robust electrically conductive water treatment membranes," 
ACS Applied Materials \& Interfaces, vol. 11, no. 11, pp. 10914-10921, 2019.

[320] A. Gupta, L. Holoidovsky, C. Thamaraiselvan et al., "Silverdoped laser-induced graphene for potent surface antibacterial activity and anti-biofilm action," Chemical Communications, vol. 55, no. 48, pp. 6890-6893, 2019.

[321] "DeZeen Guardian G-Volt masks would use graphene and electrical charge to repel viruses and bacteria," https://www .dezeen.com/2020/03/06/guardian-g-volt-face-maskgraphene-coronavirus-bacteria/.

[322] M. R. Ricci, "Ultra-violet germicidal mask system," US Patent US5165395, 1992.

[323] R. Liao, "Fitbit's Chinese rival Amazfit mulls a transparent, self-disinfecting mask," https://techcrunch.com/2020/05/17/ fitbits-rival-amazfit-transparent-reusable-mask/.

[324] S. Sheth, "This air purifying face-shield creates a bubble of safety around its wearer | Yanko Design," https://www .yankodesign.com/2020/04/30/this-air-purifying-faceshield-creates-a-bubble-of-safety-around-its-wearer/?utm_ source $=$ feedburner\&utm_medium $=$ feed\&utm_campaign $=$ Feed\%3 A+yankodesign+\%28Yanko+Design+-+Form +Beyond+Function $\% 29$.

[325] R. Thukral, "Face mask designed for a surreal future where wearing PPE is humanity's new norm | Yanko Design," https://www.yankodesign.com/2020/05/03/face-maskdesigned-for-a-surreal-future-where-wearing-ppe-ishumanitys-new-norm/.

[326] L. Leonardo, "Dyson patents a face mask built into a pair of headphones," https://www.gadgetmatch.com/dyson-facemask-air-purifier-headphones-patent/.

[327] S. B. Courtney, J. Mackey, N. Matthew, and J. Jennings, “A wearable air purifier," US Patent GB2575812, 2018.

[328] F. Gder, A. Ainla, J. Redston et al., "Paper-based electrical respiration sensor," Angewandte Chemie International Edition, vol. 55, no. 19, pp. 5727-5732, 2016.

[329] M. C. Caccami and M. Y. S. Mulla, "Graphene oxide-based radiofrequency identification wearable sensor for breath monitoring," IET Microwaves, Antennas and Propagation, vol. 12, no. 4, pp. 467-471, 2018.

[330] M.-H. Seo, H.-H. Yang, K.-W. Choi, J.-S. Lee, and J.-B. Yoon, "A simple breathing rate-sensing method exploiting a temporarily condensed water layer formed on an oxidized surface," Applied Physics Letters, vol. 106, no. 5, article 053701, 2015.

[331] “Davis, L. f. E. a. P. S. L.-U. o. C. Smart mask project,” http:// lepsucd.com/smart-mask-project/.

[332] T. Zhao, Q. Tao, and H. Liu, "Smart mask, method, smart mask and apparatus for calculating pollutant absorption quantity," US Patent US20170028231A1, 2020.

[333] Y. Cheng, C. Wang, J. Zhong et al., "Electrospun polyetherimide electret nonwoven for bi-functional smart face mask," Nano Energy, vol. 34, pp. 562-569, 2017.

[334] C. M. Williams, M. Abdulwhhab, and S. S. Birring, "Exhaled Mycobacterium tuberculosis output and detection of subclinical disease by face-mask sampling: prospective observational studies," The Lancet Infectious Diseases, vol. 20, no. 5, pp. 607-617, 2020.

[335] A. MacDonald, "Mask sampling for COVID-19 detection | technology networks," https://www.technologynetworks .com/diagnostics/articles/mask-sampling-for-covid-19detection-333724.
[336] A. Bendix, "Harvard and MIT researchers are developing a face mask that lights up when it detects the coronavirus," https://www.businessinsider.sg/coronavirus-face-masklight-up-screening-tool-test-2020-5.

[337] A. L. Allison, E. Ambrose-Dempster, T. D. Aparsi et al., The environmental dangers of employing single-use face masks as part of a COVID-19 exit strategy, UCL Open: Environment Preprint, 2020.

[338] "Coronavirus: French alarm at Covid-linked Med pollution," https://www.bbc.com/news/world-europe-52807526.

[339] J. Zheng and S. Suh, "Strategies to reduce the global carbon footprint of plastics," Nature Climate Change, vol. 9, no. 5, pp. 374-378, 2019.

[340] “The science of a DIY mask," https://www.a-star.edu.sg/ News-and-Events/a-star-news/news/covid-19/the-scienceof-a-diy-mask.

[341] J. Jung, "KAIST researchers develop highly reusable mask filter," https://www.koreatechtoday.com/kaist-researchersdevelop-highly-reusable-mask-filter/.

[342] I. Krucińska, W. Strzembosz, K. Majchrzycka, A. Brochocka, and K. Sulak, "Biodegradable particle filtering half-masks for respiratory protection," Fibres \& Textiles in Eastern Europe, vol. 96, pp. 77-83, 2012.

[343] J. Zhang, S. Gong, C. Wang, D.-Y. Jeong, Z. L. Wang, and K. Ren, "Biodegradable electrospun poly(lactic acid) nanofibers for effective $\mathrm{PM}_{2.5}$ removal," Macromolecular Materials and Engineering, vol. 304, no. 10, article 1900259, 2019.

[344] O. Guzdemir and A. A. Ogale, "Influence of spinning temperature and filler content on the properties of melt-spun soy flour/polypropylene fibers," Fibers, vol. 7, no. 10, p. 83, 2019.

[345] L. Corpuz-Bosshart, "UBC researchers develop biodegradable medical mask for COVID-19," https://news.ubc.ca/ 2020/05/21/ubc-researchers-develop-biodegradablemedical-mask-for-covid-19/. 